\title{
MONTE CARLO SIMULATION OF NONLINEAR RADIATION \\ INDUCED PLASMAS
}

BY

BENJAMIN SHAW-hu WANG

B.S., National Taiwan University, 1960

M.S., University of Missouri (Rolla), 1964

\section{THESIS}

Submitted in partial fulfillment of the requirements for the degree of Doctor of Philosophy in Computer Science in the Graduate College of the

University of Illinois at Urbana-Champaign, 1972

\author{
Urbana, Illinois
}

\section{NOTICE}

This report was prepared as an account of work sponsored by the United States Government. Neither the United States nor the United States Atomic Energy Cominision, nor any of their employees, nor any of Comission, nor any their contractors, subcontractors, or their makes any warranty, express or implied, or assumes any legal liability or responsibility for the accuracy, completeness or usefulness of any information, apparatus, product or process disclosed, or represents that its use would not infringe privately owned rights. 


\section{DISCLAIMER}

This report was prepared as an account of work sponsored by an agency of the United States Government. Neither the United States Government nor any agency Thereof, nor any of their employees, makes any warranty, express or implied, or assumes any legal liability or responsibility for the accuracy, completeness, or usefulness of any information, apparatus, product, or process disclosed, or represents that its use would not infringe privately owned rights. Reference herein to any specific commercial product, process, or service by trade name, trademark, manufacturer, or otherwise does not necessarily constitute or imply its endorsement, recommendation, or favoring by the United States Government or any agency thereof. The views and opinions of authors expressed herein do not necessarily state or reflect those of the United States Government or any agency thereof. 


\section{DISCLAIMER}

Portions of this document may be illegible in electronic image products. Images are produced from the best available original document. 
MONTE CARLO SIMULATION OF NONLINEAR RADIATION

INDUCED PLASMAS

Benjamin Shaw-hu Wang, Ph.D.

Department of Computer Science

University of Illinois at Urbana-Champaign, 1972

A Monte Carlo simulation model for radiation induced plasmas with nonlinear properties due to recombination has been developed, employing a piecewise linearized predictcorrect iterative technique. Several important variance reduction techniques have been developed and incorporated into the model, including an antithetic variates technique (negatively correlated variates) which has proven to be most useful. This approach is especially efficient for plasma systems with inhomogeneous media, multidimensions, and irregular boundaries, where other analytic or numerical solutions are either unrealistic or impractical. This model is quite general in scope and should be applicable to a variety of similar particle transport and diffusion-type plasma problems.

The Monte Carlo code developed in this work has been applied to the determination of the electron energy distribution function and related parameters for a noble gas plasma created by $\alpha$-particle irradiation. The radiation induced plasma involved was characterized by the following features:

1) a continuous internal volume-source of high energy electrons 
2) an applied electric field (optional)

3) secondary electron production via ionization processes

4) losses via recombination and leakage across boundaries

The calculations were specifically carried out for a helium gas medium with an electron source rate from $10^{14}$ to $10^{22}$ $\left(\# / \mathrm{cm}^{3}-\mathrm{sec}\right)$, initial electron energies from 70 to $1000 \mathrm{eV}$, pressures from 10 to 760 tcrrs and E/P (electric field/pressure) ratios from 1 to $10 \mathrm{~V} / \mathrm{cm}$-torr.

For the lower source rates, it is observed that, in the zero electric-field case, the low-energy portion of the distribution function is quite close to a standard Maxwellian distribution. However, the high-energy portion (above the ionization potential) of the distributions is a rapidly decaying parabolic-shaped tail which can be crudely represented by a $1 /$ (Energy) distribution. The addition of the electric field causes the distribution function in the lowenergy region to shift towards Smit's (or Druyvesteyn's) distribution while the high-energy protion essentially. retains its zero field shape. As the source rate increases beyond certain value (roughly $10^{18} \mathrm{~cm}^{-3}-\mathrm{sec}^{-1}$ in the present case), the low-energy region begins to depart from the standard Maxwellian or Smit distributions and this departure can become quite large. 
A major emphasis in this development was placed on computation efficiency. The present computation scheme with its variance and accelerated convergence techniques is thought to be quite efficient compared to alternate approaches . 
ACKNOWLED GEMENT

The author wishes to express his sincere appreciation and gratitude to Professor George H. Miley for his patient guidance and invaluable help throughout the course of this work, without which this study would have been impossible. The author wishes to also thank Mr. Ronne Lo for his most valuable and helpful discussions.

The author would also like to thank Professor J. N. Snyder, the head of Computer Science Department, and the Nuclear Engineering Program for their financial support. This work was supported in part by the Physics Branch of the AEC Research Division under Grant No. AT(II-1)-2007 and by the NASA-AEC Space Power Office under Gxant No. NGR 14-005-183.

Finally, the author wishes to thank Mrs. Sharon Taylor for her excellent typing of the final thesis manuscript. 
TABLE OF CONTENTS

CHAPTER

Page

I. INTRODUCTION ....................... I

A. Statement of the Problem and Objectives . I

B. The Importance of Nuclear Radiation

Produced Plasmas and the Physical Model . I

C. General Approaches and Methods of

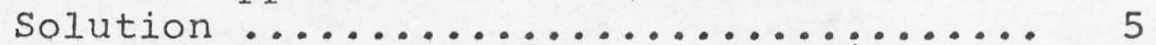

II. THE MATHEMATICAI MODEL: NUMERICAL SCHEME

AND PHYSICAL PROBLEM ................. 8

A. General Description of the Physics of

the problem .................... 8

1. Physical Processes Involved ........ 8 8

2. Relation to the Transport Equation ... 10

B. The Stochastic Model - Numerical Scheme . 12

1. The Geometry and the Coordinate

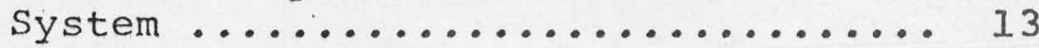

2. Tracing Procedure ............... 20

a. The System Without Electric

Field $\left(E_{f}=0\right) \ldots \ldots \ldots \ldots . \ldots . . . . .21$

b. With Electric Field Present ..... 25

c. Types of Collisions and Cross

Sections .................. 27

3. The Prediction and Correction of Dominant System Parameters ........ 29

III. VARIANCE REDUCTION TECHNIQUES ........... 33

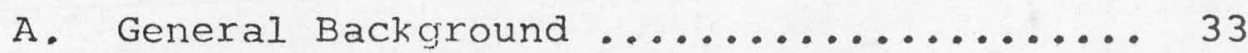

B. Importance sampling ................ 38

C. Russian Roulette and Splitting ......... 42

D. Initial Guess ................... 44

E. Antithetic Variates ................. 46

F. Double History (Double Processing) 
CHAPTER

Page

IV. MONTE CARLO SIMULATION CODE ............. 52

A. Introduction ................... 52

B. Calculational Procedure and Program

Description .................... 52

V. $\operatorname{RESULTS} \ldots \ldots \ldots \ldots \ldots \ldots \ldots \ldots \ldots \ldots \ldots \ldots \ldots . \ldots \ldots \ldots$

A. Convergence and Reliability ...........66 63

B. Error Estimation and Analysis ......... 64

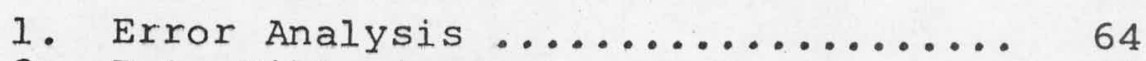



C. Soluttions of the Problem ............. 71

1. Preliminary Checks .............. 71

2. The Electron Energy Distribution Function without Electric Field .... 77

3. The Electron Energy Distribution Function with an Applied Electric Field ....................... 90

D. Conclusion on Monte Carlo Results ...... 104

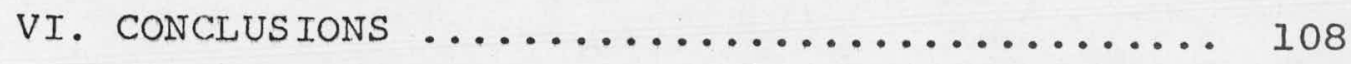

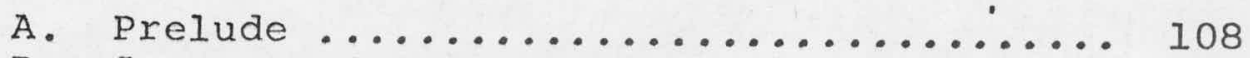

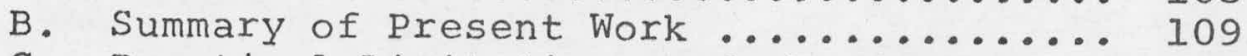

C. Practical Limitations and the Cures .... 112

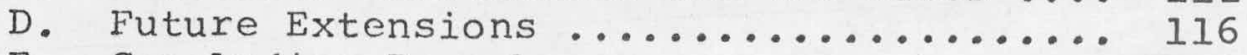

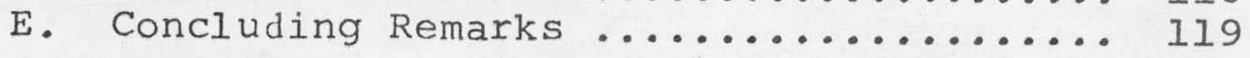

LIST OF REFERENCES ............................. I I

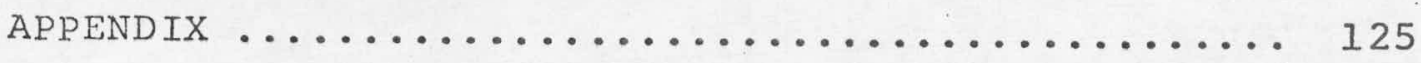

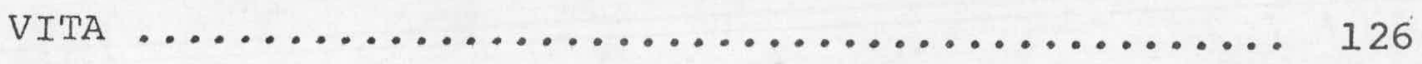




\section{CHAP TER I}

\section{INTRODUCTION}

\section{A. Statement of the Problem and Objectives}

Two major goals were set for this study. The first was to develop an efficient Monte Carlo simulation technique for solving radiation-induced plasma problems. This required adapting several variance reduction techniques in order to increase the Monte Carlo efficiency. The second goal was to apply the model developed herein to study the characteristics of a noble-gas plasma created by alpha-particle irradiation. The major point of interest is the determination of the electron energy distribution in the nuclear radiation induced plasma. This distribution is of direct importance to current experimental studies at the University of IIIinois where the possibility of directly pumping* gas lasers with nuclear radiation is under study (6). Recent results from these studies also show that nuclear radiation can be used to enhance the output from some type of electrically pumped lasers (5).

B. The Importance of Nuclear Radiation produced Plasmas Nuclear radiation can be used in two ways: to directly pump the laser, or alternately, where an electric discharge

* "Pumping" of a laser is definec as the introduction of enexgy into the lasing medium required for population inversion. The electrical current which creates the discharge in the conventional gas laser is the pump in that case. Flash lamps are used for many solid state lasers. 
serves as the main pump, to enhance the operation of lasers. The possibility of efficient coupling of lasers and nuclear reactors has generated an interest in the use of high-energy ion beams to pump lasers. To avoid radiation damage problems, gas lasers are generally thought of in this connection. There are several important reasons for searching for more direct methods of coupling energy from reactor sources into lasers (8). One is the possibility that the resulting laser may offer unique characteristics, e.g., new frequencies and new methods of modulation and control. Simplified coupling could also offer weight reductions and improved reliability, which may be of advantage in space applications. Also, it may eventually become feasible to simultaneously extract several energy forms, e.g., laser and electrical power from a single nuclear station.

In most gas lasers the excited states, and consequently the inversion of population necessary for laser operation, are formed directly or indirectly by electronic collisions in a plasma generated by an electrical discharge. However, an alternate way to accomplish this goal is via nuclear radiation which can produce ionization and excitation through both direct interactions and interactions due to secondaryelectron production. Given the high neutron fluxes available from nuclear reactors, the particles from neutron-induced nuclear reactions (e.g., a-particles from a Boron coating on the wall of a laser tube) can furnish the nuclear radiation 
source. The description of the transport of both the primary radiation and the secondary electrons, from their point of birth throughout their path in the gas, is a key problem in the quantitative evaluation of the plasma. Therefore the development of nuclear.-radiation pumping requires a thorough understanding of the radiation-induced plasma.

The following physical model is applied to the situation of a radiation-induced plasma. Pertinent quantities of interest include: electron energy distribution spectrum; average electron energy; and rate of energy loss.

The heavy-charged particles (a-particles) are born in coating and enter an adjacent gas. In the present calculations, the cylindrical geometry of the actual laser tube is approximated by a slab geometry (cf. Figure l) with two parallel plane boron coatings separated by a gaseous medium. The a-particle radiation continuously creates high energy electrons throughout the gas volume (with energy $E_{0}$ ). The resulting plasma is a weakly ionized, three-component plasma (electrons, positive ions, and neutral gas atoms). It is assumed that the alpha-particle trajectory can be treated analytically using methods such as developed in (7). Further, this reference shows how to obtain the primary secondary-electron ( $\delta$-ray) energy spectrum. The difficult problem, however, is to follow the thermalization and further secondary production of these primary electrons. 
Thus, the Monte Carlo simulation starts off by following these electrons on their life time histories, i.e., by simulating the course of their thermalization. Electrons go through various processes of collisions and scattering, resulting in a complex process of diffusion, energy degradation and secondary production, but finally, they recombine with positive ions or escape from the system, ending their histories.

With the radiation rates of interest here, the plasma is only weakly ionized and electrons mostly thermalize by collisions with the neutral background-gas atoms. In other words, collisions with ions and other free, electrons can be neglected. In this sense, the problem is simplified in that it remains linear. However, some non-linearity enters in the following way. The recombination process depends on the ion density and spatial distribution. However, the ions are originally formed by ionizations which occur as the electrons slow down.

Another important characteristic is that the plasma is highly non-equilibrium. The thermalized electrons and ions form an approximately Maxwellian background, but superimposed on this is the high energy tail due to the electrons in the process of slowing. Also, the alpha particles themselves represent a non-equilibrium component.

In summary, the special features of the plasma system are: 
1) Continuous internal volume source.

2) An applied electric field may or may not be present.

3) Finite cylindrical geometry--approximated here by slab geometry.

4) Electron-ion pair production and recombination processes.

5) The energy range of the electrons range from kilovolts to thermal (ev.):

6) The system represents a non-equilibrium plasma, but in a steady-state condition (or stationary).

7) Due to relatively small densities of electrons and ions compared with that of gas atoms, direct interactions between the electrons themselves or electron-ion collisions are neglected.

C. General Approaches and Methods of Solution

Possible approaches toward solutions of these types of problems (particle transport and diffusion) can be categorized as follows:

1) Analytical. solution of the governing Boltzmann transport equation.

2) Numerical solution of the Boltzmann transport equation.

3) Direct analog simulation (Monte Carlo).

4) Mixtures of the above approaches.

The governing equation for this type of problem is the Boltzmann equation, and most classical methods have involved approximate analytic solutions of it. Solutions are possible only after various approximations and simplifications are made, and such solutions are often very restrictive and/or difficult to extend to actual situations of interest. More 
or less the same drawbacks apply to numerical solutions. However, in this case approximations are necessary or the computation time becomes too long, especially as the dimensionality and the complexity of the problem increases. In fact, the computation time increases roughly exponentially with the dimensionality of the problem. Usually the governing partial differential and integral equations (coupled Boltzmann equations) are reduced to finite difference equations, and the requirements for stability and convergence severely limit the maximum step sizes of the independent variables. Further difficulties with the practical application of such approaches arise because it is often difficult to include realistic boundary conditions.

Direct simulation by the application of Monte Carlo techniques seems to be the better approach; sometimes the only approach for such problems. It by-passes the direct solution of the partial differential-integral equation, and generally no approximations and/or simplifications are necessary. Complicated boundary conditions can be handled easily, and the computation time increases at the most linearly with the dimensionality of the problem. However, computer time still may be a problem so that judicious selection of numerical schemes and variance reduction techniques are required.

Many methods have been devised in the past which involve a mixture of analytic, numerical, and Monte Carlo approaches. 
In such situations, Monte Carlo is usually applied to the most difficult part of the problem. For instance, in the evaluation of the non-linear, five-dimensional collision integral in the Boltzmann equation, straight forward numerical quadrature would require months of computation time even on the present day fast computers. The Monte Carlo (statistical sampling techniques which closely resemble the actual collision phenomena) techniques can produce results within reasonable computation time (24). The Monte Carlo method may be defined as a numerical device (numerical experimentation) for studying an artificial stochastic model of a physical or mathematical process. This study may be approached by one of two methods. The first is the process of simulation (direct analog simulation) in which the particles - electrons and ions in the case of plasma systems, or photons in the case of radiative transfer - are represented by a game of chance in the computer. In the second method, which is non-simulation, the Monte Carlo technique is strictly used as a numerical stochastic device for the solution of a given integral or partial differential equations $(24,25)$. 
CHAPTER II

THE MATHEMATICAL MODEL: NUMERICAL SCHEME AND PHYSICAL PROBLEM

A. General Description of the Physics of the Problem

The plasma under study is a weakly-ionized threecomponent plasma, and is non-equilibrium as described in Chapter I. The $\alpha$-particle radiation continuously creates high-energy electrons throughout the gas volume. The Monte Carlo simulation is to trace these electrons' life histories and their complex processes of diffusion and energy degradation. Since the diffusion process of the electrons through the medium is affected by the density of the positive ions and the other electrons present (background electrons), the resulting problem is non-linear.

\section{Physical Processes Involved}

The mechanisms by which particles interact with the atoms of the medium are elastic and inelastic collisions, by which the incident particle is slowed and dissipates some or all of its kinetic energy to the medium by the combined action of the above mentioned processes. The particle can also experience so called superelastic collision by which the particle collides with the excited atoms gaining some energy. Finally, the particle can collide and recombine with positive ions or can leak out of the system thus ending its life 
history.

As described in Chapter I, the type of interaction which is involved in any particular collision is governed by the laws of chance. For large numbers of interactions, the frequency of occurrence of any given interaction is determined by the relative magnitudes of the interaction cross sections. Secondary knock-on electrons due to the ionization process may be capable of further interacting with the atoms and producing additional secondary electrons.

As the system reaches steady-state, a balance is reached such that the birth rate for new particles, i.e., the rate at which primary or source electrons enter the system plus the production rate of secondary electrons due to ionization should approximately equal the loss rate due to leakage and absorption or recombination.

Since the transport of radiation through a medium affects its absorption properties, the transport of individual particles is indirectly affected by the density of the other particles present, and this introduces some nonlinearities into the system. We shall adopt a piecewise predict-correct technique to approximate the nonlinear system with a linear model.

We are concerned with the simulation, by random sampling of the scattering of the charged particles. The direct simulation of the physical scattering processes in such a way that the diffusion process is imitated by letting 
the particles carry out a random walk, each step of which takes into account the combined effect of many collisions. The mechanisms by which electrons interact with the atoms of the medium are elastic, inelastic, and superelastic collisions as well as recombination with positive ions. The incident source electron is slowed and dissipates some or all of its energy to the medium by the combined action of the various scattering processes, until it escapes, or recombines with an ion (radiative energy losses are neglected). The probability that an interaction occurs is determined by the total collision cross-section. The type of interaction is then determined by the relative magnitudes of the individual cross-sections.

2. Relation to the Transport Equation

Although no direct use will be made of the governing Boltzmann transport equation in the sequel, we shall write it down briefly, in order to indicate the mathematical problems to be solved implicitly by the Monte Carlo method. It is a nonlinear integro-differential equation of the form

$\frac{\partial f}{\partial t}+\bar{u} \cdot \nabla_{x} f-\frac{l e y}{m}(\bar{E}+\bar{u} \times \bar{B}) \cdot \nabla_{u} f=\left(\frac{\partial f}{\partial t}\right)$ collisions

where f: number density distribution function

$\bar{B}$ : magnetic field

$\bar{u}$ : velocity of the particles

$\overline{\mathrm{E}}$ : electric field strength 


$$
\begin{aligned}
& t: \text { time } \\
& r: \text { spatial variable }
\end{aligned}
$$

In our situation $\bar{B}=0$ since the magnetic field does not exist. Further, if we assume an infinite medium and uniform source, $\nabla_{x} f=0$, the distribution function $f$ depends only on energy. Then the above equation reduces to

$$
\frac{\partial f}{\partial t}-\underset{m}{\underline{E}} \cdot \nabla_{u} f=\left(\frac{\partial f}{\partial t}\right) \text { collisions }
$$

The right hand side term is the collision term, which is the most difficult part of the equation. The effects of all relevant particle interactions must be included in this term. For the case of electron collisions with stationary gas atoms of uniform density, we can write the collision term as:

$$
\left(\frac{\partial f}{\partial t}\right)_{\text {collision }}=\left(\frac{d N_{\text {in }}-d N_{\text {out }}}{d V \cdot d t}\right)
$$

where dN ${ }_{\text {in }}$ gives the number of phase space points (electrons) that are scattered into the phase-space volume dV by collision in time dt. Similarly, dN out represents the number of points scattered out of $d V$ by collisions in time dt. This Eq. 2 states that the change in the distribution function $f$ are caused by collisions of various kinds and the external force due to the electric field.

The assumptions that enter into the derivation of the transport equation which carry over to the Monte Carlo 
simulation include:

a) the scattering centers (background atoms and ions) are distributed at random, i.e., possible correlations between the positions of various atoms and ions are not taken into account.

b) In the absence of an electric field, the trajectory of the particle is idealized as a zig-zag path, consisting of flights interrupted by sudden collisions in which the energy and direction of the particle changed. The quantummechamical interference (electron diffration) resulting from the coherent scattering by several centers are neglected, and a particle, in the course of traversing the medium, interacts with one scattering center at a time.

The diffusion process in terms of the transport equation is analogous to the use of Eulerian coordinates in hydrodynamics, in that one asks about the flux at given points in space. By contrast, the Monte Carlo method uses Lagrangian coordinates; one attaches a label to a particular bit of fluid, i.e., a diffusing particle, and follows its history. Then, by sampling many histories, one is able, at least in principle, to solve any diffusion problem.

B. The Stochastic Model - Numerical Scheme

The essential approach to the nonlinear problem adopted here is the use of piecewise linearized steps. In each such step (an amount of time $\Delta t$ along the time axis), the 
dominate parameters, which characterize the nonlinear properties, are predicted and considered to be fixed with respect to the independent variables. During the time $\Delta t$, the system can be considered as more or less linear, so the conventional Monte Carlo simulation technique is used. The quantities pertaining to the given system are computed for each of a reasonably large number of independent particle histories. The desired parameter values are obtained by averaging the results over all of the histories. At the end of the step, the dominate parameters are corrected before beginning the next step. In each step (time interval $\Delta t$, or cycle), instead of computing the trajectory and angular deflection of an electron after each interaction, we perform a random walk calculation. Since each step of the random walk takes into account the combined effects of many individual interactions, we form a so-called condensed history by this approach. Let us describe the model in some detail, step by step.

1. The Geometry and the Coordinate System

The initial spatial geometry in this problem is a onedimensional, finite slab (from $z=0$ to $z=D$ ) which is subdivided into uniform intervals called zones as shown in Figure 1. The energy range is from 0 to $E_{0}(=l \mathrm{keV})$ which is also subdivided into three regions: $0 \sim 1$ (thermal energy region), I to ED2, and ED2 to $E_{0}$. Each of these 
14

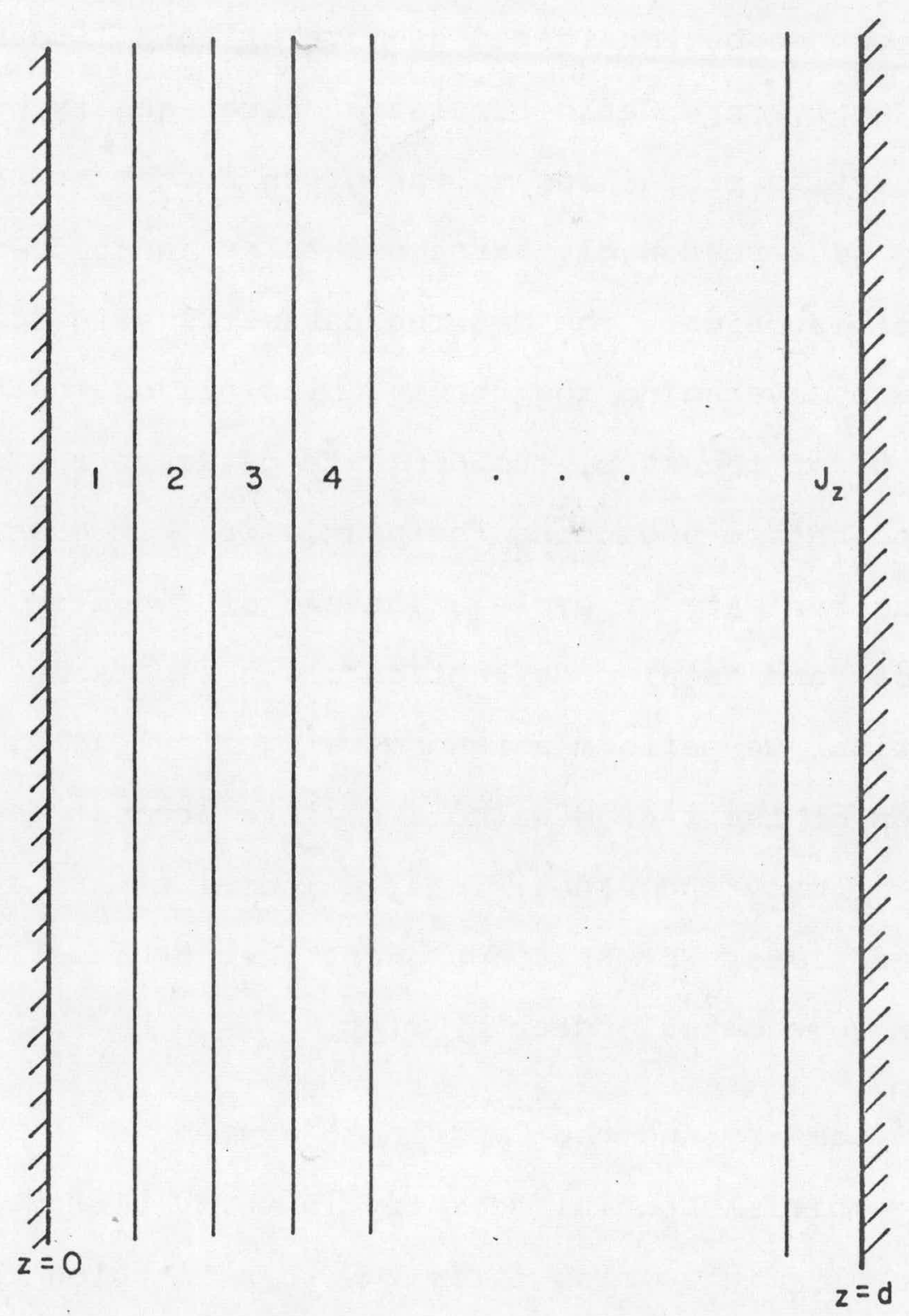

Figure 1. Slab geometry of the medium with subdivided zones. 
regions is further divided into smaller uniform or logarithmic spacing (intervals). The particle number density distribution $f(E, z)$ is a function of energy $E$ and spatial position $z$ and is generated such that particles with energy and spatial posjtion falling into the same intervals are grouped together and normalized by the total particles processed. Although the system is stationary, we deliberately introduce the time variable for the convenience of recording output information and carrying out the calculation steps. The time serves as a clock, or we can imagine that the system begins in a transient state. Then, as time goes on it will eventually reach steady state. The storage of the timing information for individual particles is not necessary. Instcad of following a single particle from the beginning to the end of its history, we follow a large number of particles, say $\mathbb{N}$ (which represent the sample particles of the whole system population) through a small time interval at (called the cycle time). Within this time interval, the system is considered to be linear, and the particles are processed more or less independently as in a conventional linear Monte Carlo model. At the end of $\Delta t$, called census time, we record data information for each particle (rccord particle state information). During this time interval (or present time cycle) some particles may experience collisions of some kind, some may not, and new particles (source particles, secondary particles produced 
by ionization) enter the system. Some particles may be absorbed or escape from the system; their histories are therefore ended. This represents a random walk calculation, and each step of the random walk takes into account the combined effect of many individual interactions. The trajectory of a particle which is computed by this approach is called a condensed history. The residue particles (ionization produced secondary particles) are also set into motion, and their histories are followed separately. They are like all the other source particles in all respects. The complete description of particle histories is approximated by the condensed histories, or we take sequences of snapshots of all the particles in the system at various times to provide a moving picture of the history that can be used to estimate the quantities of interest. The path lengths travelled by the particles are also used as a clock to measure time, or equivalently, the variable $t$ may be replaced by $S(t)=\int_{t_{0}}^{t} v(x) d x$. The trajectories of the particles (the condensed histories) are described by the arrays as indicated in Figured 2. The superscript denotes the cycle time number; the subscript denotes particle number where the index 0 refers to the initial state of the particle. Other notation includes $\mathrm{E}$ : the particle energy, $z$ : spatial position, and $U$ : the direction, and wt: the weight. When the particle has traveled a pathlength DCFN (in the same duration $\Delta t$ ), a condensed history 
$1^{t}$
$t_{0} \quad t_{1}$
$t_{j}$
$t_{m}$

$E_{1}^{0} \quad E_{1}^{1} \quad \cdots \quad E_{1}^{j} \quad \cdots . . \quad E_{1}^{m}$

$1 \quad z_{1}^{0} \quad z_{1}^{1}$

$z_{1}^{j}$

$\mathrm{z}_{1}^{\mathrm{m}}$

$\mathrm{U}_{1}^{0} \quad \mathrm{U}_{1}^{1}$

$u_{1}^{j}$

$u_{1}^{m}$

$w t_{1}^{0} \quad w t_{1}^{1}$

$w t_{1}^{j}$

$w t_{1}^{m}$

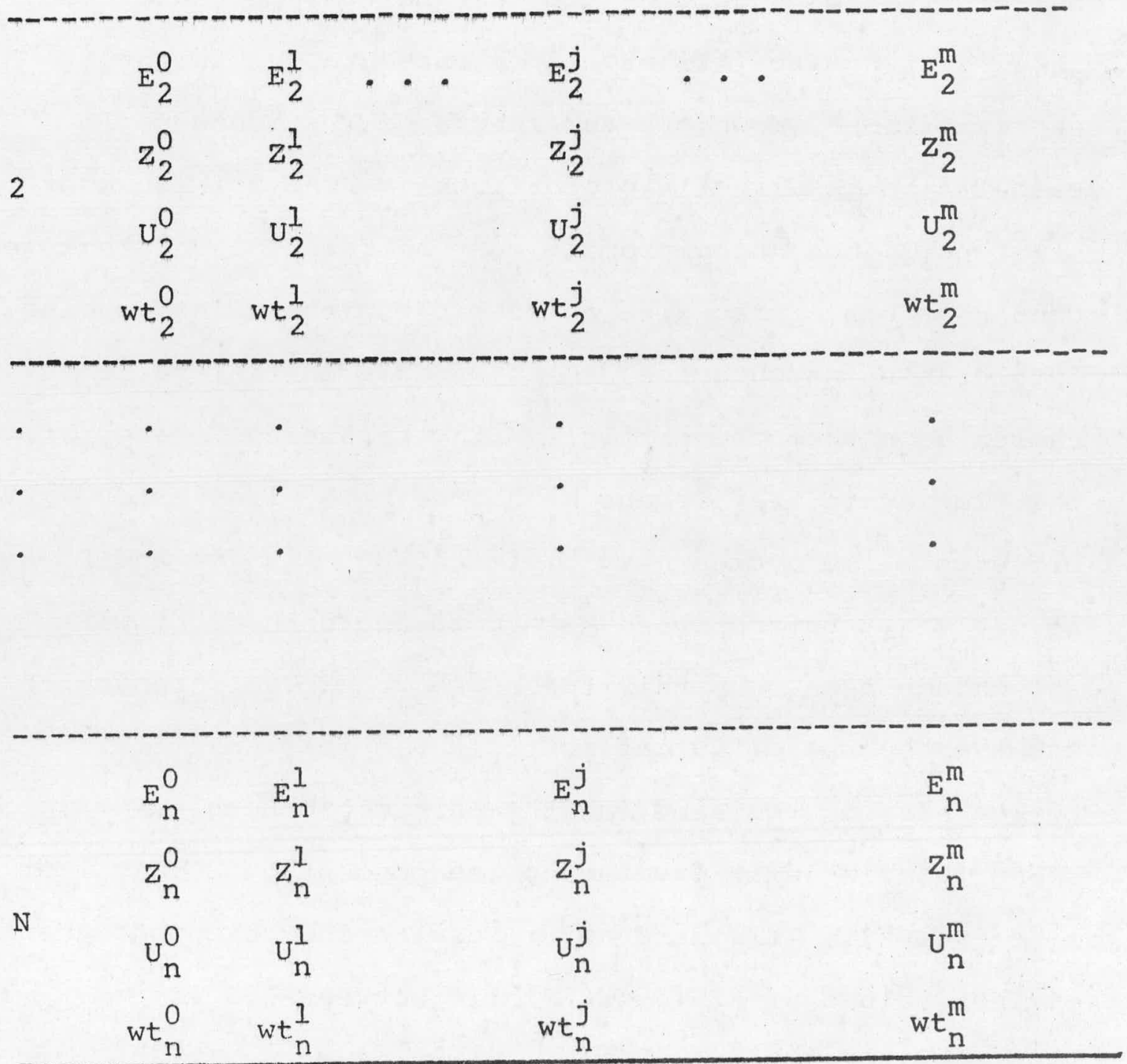

Figure 2. Snapshops of the particles' condensed histories. 
is sampled. Letting the particle carry out a random walk in each step ( $f$ Iom state $M$ to state $M+l$ ) takes into account the combined effect of many collisions. The sizes of the steps of the random walk, i.e., the pathlength intervals DCEN (equivalent to $\Delta t$ ) are chosen such that the total number of steps should be kept as small as possible. Too large a step size ( $\Delta t)$ may cause inaccuracies in the approximation. However, the length of the Monte Carlo calculation is directly proportional to the total number of steps. Thus, a compromise must be reached such that we choose as big a step size as possible without introducing intolerable inaccuracies. A reasonable initial value is chosen such that the particle with the average energy of the time cycle travels one mean free path, i.e., $\Delta t=D_{a v g} / V_{a v g}{ }^{*}$ Diffexent time cycles have different $E_{\text {avg }}\left(V_{\text {avg }}\right.$ ) and $D_{\text {avg }}$ so $\wedge t$ may vary, but as steady-state is approached, $\Delta t$ will not change much. By this technique we not only randomize and average the different particle samples, ensemblewise, but we also do the same (on the particle ensemble sets) along the time axis (averaging independent but equivalent time cycles). Trial and error results indicate that the optimum values of $\Delta t$ (or DCEN) lie between 0.5 ( $\mathrm{D}_{\mathrm{avg}} / \mathrm{V}_{\mathrm{avg}}$ ) $2.0\left(D_{\text {avg }} / V_{\text {avg }}\right)$.

The maximum number of particles $(N)$ that can be processed in each cycle is limited by the amount of storage available in the computer system. For the IBM 360/75 system 
at the Illinois Computer Center, the storage of the data for 5,000 particles is easily accomplished. By playing Russian roulette, splitting, or similar techniques, the total number of artificial particles (N) can be increased by an order of magnitude. The end of one time cycle $t_{i}$ is the start of the next time cycle $t_{i+1}$. While keeping track of the particles in a current time cycle at one time, we simultaneously record the data information after each particle reaches the census and extract the output parameter values after all the particles have been processed. In this way the storage requirements are reduced to a minimum level possible.

The limitation on $\mathrm{N}$ by the storage requirement may be further relaxed by the following technique. Instead of recording and tallying the output information and clearing or reinitializing all parameter values at the end of every cycle, we record the accumulated information for $\mathrm{K}$ continuous, and similar but statistically independent time cycles, then renormalize and reinitialize. In this way the total number of particles processed is $\mathrm{KN}$, and if processing of a large number of particles is desired, we can use a larger value of $K(K>1)$ at the expense of more processing time. The reason for this is based on the fact that the system being simulated is time independent. We have introduced the time variable artificially so that ergodicity holds, namely ensemble averaging is the same as time averaging. 


\section{Tracing procedure}

The simulation is initialized by introducing $\mathrm{N}$ particles with energy $E_{\text {in }}$ [sampled from an initial guess for the number density distribution vs, energy], with spatial position $z_{i}^{0}$ (uniformally distributed throughout the spatial region), with direction $u_{i}^{0}(u=\cos \theta ; \theta$ : angle of flight with $z$ axis) which is uniformly distributed between -1 and +1 and with weight $w t_{i}^{0}$. Volume particles are emitted uniformly throughout the medium and isotropically in direction; namely, at $t=t_{0}$ :

$$
\begin{aligned}
& E_{i}^{0}=E_{i n} \\
& z_{i}^{0}=r_{i} \cdot D \\
& u_{i}^{0}=2 r_{i}-1
\end{aligned}
$$

where $r_{i}$ : random number, uniformly distributed between 0 ,

$$
1 \text {, or } r_{i} \in(0,1)
$$

a: slab width or cylinder diameter

$w t_{i}, E_{i n}$ : weight and particle energy determined from initial distribution.

once the particle data are determined, the geometry routine is entered. This includes the determination of the distance to boundary $d_{B}$, the distance to collision $d_{c o l}$, and the distance to census time $a_{c e n}$ (clock distance equivalent to $\Delta t$ ). These distances are defined by: 


$$
\begin{aligned}
& \begin{aligned}
d_{B} & =[(j+I) \Delta z-z] / u \text { if } u>0 \\
& =(z-j \wedge z) /|u| \text { if } u<0 \\
& =D L B(a \text { large number } D L B>\Delta z) \text { if } u \approx 0
\end{aligned} \\
& d_{\text {Col }}=\lambda(E)|\ln r|=|\operatorname{Inr}| / \sum_{t}(E) \\
& d_{c e n}=D_{\text {avg }} \cdot D I=V_{\text {avg }} \cdot \Lambda t \cdot D I .
\end{aligned}
$$

where $r$ : random number uniformly distributed

$j:$ zone number

$\Delta z:$ spatial interval size

$\Sigma_{t}: \begin{aligned} & \text { the total cross-section evaluated at the particles' } \\ & \text { energy }\end{aligned}$

$D_{\text {avg: }}$ the average value of $D_{\text {col }}$ of $N$ particles of the previous cycle. It is approximately one mean free path corresponding to $\mathrm{E}_{\text {avg }}$ : the average energy of the cycle.

DI: input parameter for controlling the cycle time interval.

The next step depends on which of these distances is the smallest.

a. The System Without Electric Field ( $E_{f}=0$ )

i) If the smallest distance is $d_{c e n}$ : the particle is advanced in position and time to census time.

$$
\begin{aligned}
& z^{\prime}=z+u \cdot d_{\text {cen }} \\
& u^{\prime}=u \\
& E^{\prime}=E
\end{aligned}
$$

The particle data is stored, the energy is tallied, and 
the density counter is incremented. Then we go on to process the next particle.

ii) If the smallest distance is $d_{B}$, the particle is advanced to the boundary such that:

$$
\begin{aligned}
& z^{\prime}=z+\left(d_{B}+0.001 \cdot \Delta z\right) \cdot u \\
& j=\operatorname{INT}\left(z^{\prime} / \Delta z\right)+1 \\
& d^{\prime}{ }_{\text {COI }}=d_{\text {COI }}-d_{B} \\
& d^{\prime}{ }_{\text {cen }}=d_{\text {cen }}-d_{B}
\end{aligned}
$$

where $j$ is the zone number. If $j>\mathrm{JZ}$ or $j<1$, then the particle is considered lost or has escaped from the system, and its history is ended. Otherwise the calculation and selection is repeated until the particle reaches the census time.

iii) If $d_{c o l}$ is the smallest, the particle is first advanced to collision point and

$$
\begin{aligned}
& z^{\prime}=z+u \cdot d_{\operatorname{col}} \\
& d_{\text {cen }}=d_{\text {cen }}-d_{\text {col }}
\end{aligned}
$$

Then the type of collision is specified. For specifying the collisional processes, we divide the whole energy range into two regions. Region I: energy range 0 to $\mathrm{EDI} \mathrm{eV}$. Region II; energy range EDI eV to $\mathrm{E}_{\mathrm{o}} \mathrm{eV}$. Edl and $\mathrm{E}_{\mathrm{o}}$ are specified by input parameters and are referred to as the energy dividing line and source particle energy, respectively. 
If the particle's energy is in the range of Region I, three kinds of collisions are possible: elastic collisions, electron-ion recombination, and superelastic collisions (where electrons hitting an excited atom pick-up the excitation energy). If the particle's energy falls in Region II, it can experience four kinds of collisions; namely, elastic collisions and three types of inelastic collisions--excitation to metastable level, excitation to other levels, and ionization. For ionization, the secondary electron so produced shares energy with the primary electron, and the energy of primary electron before collision less the ionization potential is shared between the two electrons. The partition of the shared energy could be determined according to Goodrich's experimental distribution curve (31). But based upon Thomas' (1) calculation experience, the way in which the energy is partitioned does not have a strong influence on any of the calculated mean properties. Thus, for computational simplicity, as Thomas did in all his calculations, we choose the partition ratio 9:1. The secondary electrons produced via ionization are considered as new particles introduced into the system. Thus they are followed from their point of birth just like other particles. This is equivalent to the generation method for processing branched trees of samples.

The type of collision is determined according to Figure 3 using a random number (uniformly distributed 


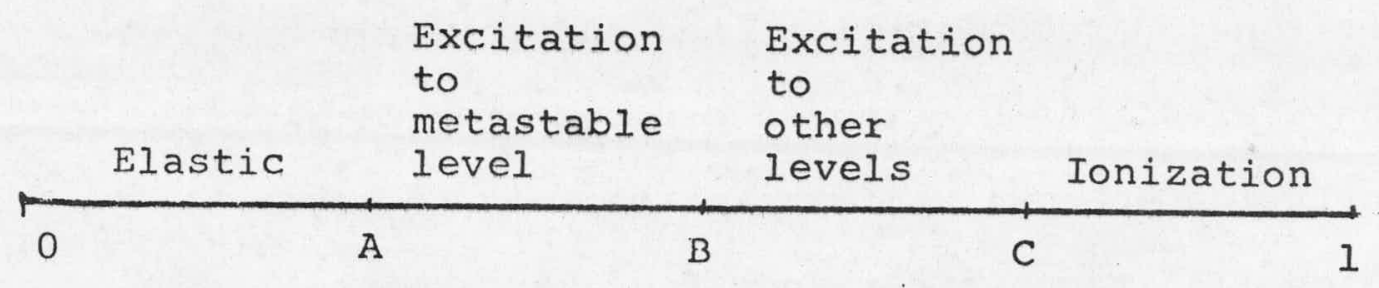

$$
\begin{aligned}
& A=P_{e l} \\
& B=P_{e l}+P_{\text {met }} \\
& C=P_{e l}+P_{\text {met }}+P_{\text {exc }} \\
& P_{\text {el }}+P_{\text {met }}+P_{\text {exc }}+P_{\text {ion }}=1
\end{aligned}
$$

Figure 3. The probability model for determining the types of collision. 
between 0 and I) to play the law of chances.

b. With Electric Field Present

The coordinate axis $z$ is defined as antiparallel to the electric field $E_{f}$. In the presence of electric field $E_{f}$, the state of motion of an electron is specified by its speed $V$ and the direction $\operatorname{cosine} \cos \theta$, where $\theta$ is the angle made by the line of flight with the z-axis at the time $t$. If an electron is in a state. $[E(V), u(\cos \theta), z, t]$, it will assume (after a flight along the orbit by $\Delta t$ ) the new state $\left[E^{\prime}\left(V^{\prime}\right), u^{\prime}(\cos \theta \cdot), z+\Delta z, t+\Delta t\right]$. These parameters are connected by the following relations:

$$
\begin{aligned}
& V^{\prime} \cdot \sin \theta^{\prime}=V \cdot \sin \theta \\
& m V^{\prime} \cos A^{\prime}=m V \cos \theta+e E_{f} \cdot \Delta t \\
& I / 2 m V^{\prime}=1 / 2 m V^{2}+e E_{f} \cdot \Delta z \\
& E^{\prime}=E+e E_{f} \cdot \Delta z
\end{aligned}
$$

where $m$ and -e are the mass and the electric charge of the electron, respectively. Here $\Delta z$ is the projection of the path length $L$ on the $z$ axis. It can be obtained as follows;

$$
\begin{aligned}
\Delta z & =\int_{0}^{\Delta z} v_{z} d t=\int_{0}^{\Delta t}\left(v \cdot u+\frac{E_{f}}{m} \Delta t\right) d t \\
& =v \cdot u \cdot \Delta t+I / 2 \cdot \frac{E_{f}}{m} \cdot \Delta t^{2}
\end{aligned}
$$

In carrying out the geometry routine, it is suitable to use time directly as the clock. The determination of the distance to collision $d_{C O l}(D C O L)$ and the distance to census 
$d_{\text {cen }}(D C E N)$ is accomplished as follows:

$$
\begin{aligned}
\text { DCOL } & =\lambda \cdot|\ln r| \\
& =2 /\left(I / \lambda(E)+1 / \lambda\left(E^{\prime}\right)^{\prime}\right) \\
\text { DCEN } & =\sqrt{V_{y}^{2}+v_{z}^{2}} \cdot \Delta t \\
V_{y} & =V \cdot \sin \theta \\
V_{z} & =v \cdot \cos \theta+\frac{E_{f}}{2 m} \Delta t
\end{aligned}
$$

where E: the initial energy of the particle

E': the energy of the particle after flight time $\Delta t$. The next step is to determine which of the distances is smaller. If DCEN $\angle \mathrm{DCOL}$, then the particle is advanced to the new state

$$
\begin{aligned}
& E^{\prime}=E+E_{f} \cdot \Delta z \\
& z^{\prime}=z+\Delta z \\
& u^{\prime}=\sqrt{E / E^{\prime}} \cdot u+e E_{f} \cdot \Delta t
\end{aligned}
$$

If $E^{\prime} \leq 0$ or $z^{\prime}>D, z^{\prime}<0$ then the particle is considered to have escaped from the system. If DCEN $\geq$ DCOL, the particle is advanced to collision point.

$$
\begin{aligned}
& \Delta t^{\prime}=\Lambda t-D C O L \cdot \Delta t / D C E N \\
& D C E N=D C E N-D C O L \\
& z^{\prime}=z+\Delta z \cdot D C O L / D C E N \\
& E^{\prime}=E+\Delta z \cdot E_{f} \cdot D C O L / D C E N
\end{aligned}
$$

then the specific collision type must be determined. 


\section{c. Types of Collisions and Cross Sections}

For the elastic collisions, the particle scatters off with angle of deflection $\theta$ and energy loss $\Delta E$, the following formula is used (36).

$$
\Delta E=\frac{2 m}{M}\left(E-E_{n}\right)\left(1-\cos \theta_{s}\right)
$$

where $A_{S}$ : scattering angle (angle of deflection)

E: particle energy

$\mathrm{E}_{\mathrm{n}}$ : target gas atom energy

$\mathrm{m}$ : electron mass

M: gas atom mass

$E_{n}$ is selected according to a Maxwellian distribution, and the $\Lambda E$ so formed may have either positive or negative values, indicating the loss or gain of energy respectively. For calculational simplicity, instead of selecting the deflection from the elaborate distributions, we use $\pi / 4$ or $\pi / 2$ deflection approximations, namely $\theta_{\mathrm{s}}=\pi / 4$ or $\pi / 2$, then $\cos \theta_{S}=0$ or 0.70711 . Another reasonable alternative is to assume that the direction after collision is equally probably in all directions (isotropic scattering). We select this direction at random with uniform distribution, and based on Eq. 23 calculate $\cos \theta_{S}$ and $\Delta E$, respectively, where

$$
\begin{aligned}
& \cos \theta=2 r_{i}^{-1} \\
& \theta_{S}=\theta-\theta_{i}
\end{aligned}
$$


where $\theta_{i}$ : incident angle

$\theta$ : outgoing angle after collision

For the three kinds of inelastic collision cross sections we use approximate formula based on experimental data as correlated by Itoh and Musha (2). The formulas are expressed in the form:

$$
\Sigma(E)=\frac{B\left(E-E_{i}\right)}{A+\left(E-E_{i}\right)^{2}}+C
$$

where the constants $A, B, C$, and $E_{i}$ depend on the kind of collision and scattering angle. For the elastic cross section in helium, we use a single approximate formula for whole effective energy range based on Heylen and Lewis's paper (10), namely:

$$
\sum_{e I}(E)=26 \exp (-0.04 \mathrm{E})\left(\mathrm{cm}^{-1}\right)
$$

For the recombination cross section for helium (35), we assume the simple form:

$$
\begin{aligned}
\sum_{R C B}(E) & =\mu(E) \cdot N^{+} \\
\mu(E) & =10^{-9} / V(E)
\end{aligned}
$$

where $\mathrm{N}^{+}$: ion density

$$
\mathrm{V} \text { : electron velocity }
$$

the superelastic cross section is

$$
\sum_{\operatorname{Sel}}(E)=\frac{0.109(19.8-E)}{0.25+(E-19.8)^{2}} \cdot \frac{N^{+}}{3 \cdot D_{n}}
$$


where $\mathrm{N}^{+}$: positive ion density

$D_{n}$ : gas atom density

$\mathrm{E}$ : electron's energy

3. The Prediction and Correction of Dominant System Parameters

There exists a single parameter, the ion density distribution $\mathrm{N}^{+}(E, z)$, which completely characterizes the nonlinearity of the system. This simplifies the problem and suggests the following predict-and-correct scheme. We assume that the average mean free path (or $\Delta t$ ) and the ion density distribution $\mathrm{N}^{+}(E, z)$ in the present time cycle also applies to the next time cycle, In other words, in each time cycle we use the predicted values of $\Delta t$ and $\mathrm{N}^{+}(\mathrm{E}, \mathrm{z})$ from previous time cycle. At the end of present time cycle, we use the up to date information to renormalize and correct the value to be used for the next time cycle. As steady state is reached, the predicted and corrected values of the parameters converge. From the continuity equation

$$
\nabla \cdot J=S-L
$$

where $J$ : the particle current flow in or out of the system

S: the source

$\mathrm{I}$ : the sum of losses

To start with, we assume that $\nabla \cdot J=0$, so that

$$
S=L=\alpha(E) N^{+}(E, z) N^{-}(E, z)
$$


Here $N^{-}(E, z)$ is the electron number density, the key quantity we are calculating. To initialize the run we have to predict $\mathrm{N}^{+}(E, z)$. It is reasonable to initially assume this distribution is uniform in $\mathrm{z}$, and a maxwellian distribution in $E$, i.e., $N^{+}(E, z)=N_{0} M(E)$. We also assume that $N^{-}(E, z)$ is the same as $N^{+}(E, z)$. Then we obtain

$$
\begin{aligned}
S=L & =\alpha(E) N^{+}(E, z) N^{-}(E, z) \\
& =\alpha(E)\left[N^{+}(E, z)\right]^{2}
\end{aligned}
$$

or $S_{0} \delta\left(E-E_{0}\right)=\alpha(E) N_{0}^{2} M^{2}(E)$

Integration of both sides gives

$$
\begin{aligned}
& S_{0}=N_{0}^{2} \int_{0}^{E_{0}} \alpha(E) M^{2}(E) d E \\
& . N_{0}=\left[S_{0} / \int_{0}^{E_{0}} \alpha(E) M^{2}(E) d E\right]^{1 / 2}
\end{aligned}
$$

This gives a starting value for the calculations. In later cycles the renormalization process involves integration of Eq. 29, with $\nabla \cdot J \neq 0$, i.e.,

$$
\begin{aligned}
& \iint \frac{d J(E, z)}{d z} d z d E=\iint S(E, z) d z d E \\
& \quad-\iint a(E) N^{+}(E, z) N^{-}(E, z) d z d E-J_{e s C}+J_{S}
\end{aligned}
$$

which reduces to

$$
\begin{aligned}
J(Z=d) & -J(z=0)=S_{0} d-\iint \alpha(E) N^{+}(E, z) N^{-}(E, z) d z d E \\
& -J_{e s c}+J_{S}
\end{aligned}
$$




$$
N_{i}^{2}=\frac{s_{0} d+J_{S}-[J(z=d)-J(z=0)]-J_{e s C}}{\iint \alpha(E) M(E) n^{-}(E, z) d z d E}
$$

where $i$ refers to ith time cycle

$$
\begin{aligned}
& \mathrm{J}_{\text {esc }}: \text { current due to escaped electrons } \\
& \mathrm{J}_{\mathrm{S}}: \begin{array}{l}
\text { current due to secondary electrons produced } \\
\text { by ionizations }
\end{array} \\
& \mathrm{M}(\mathrm{E}): \text { Maxwellian distribution (normalized) } \\
& \mathrm{n}^{-}(\mathrm{E}): \text { electron number density distribution } \\
& \text { (normalized) } \\
& \mathrm{S}_{0}: \text { source rate } \\
& \mathrm{N}_{i}: \text { ion density distribution (normalized) }
\end{aligned}
$$

The value of $\mathrm{N}_{i}$ replaces $\mathrm{N}_{0}$ in equation (33) at later time cycles. The value of $\mathrm{N}_{i}\left(\mathrm{~N}^{+}\right)$enters the formula for the recombination cross section and superelastic cross section, thus it directly affects the rates of recombination and superelastic collision. Indirectly, it affects the balance of the system, or equivalently the rate of convergence of the Monte Carlo calculation. An adaptive correction procedure is used here. It is based on the value of the "balance factor" BF, defined as the ratio of the particles introduced into the system by source and ionization processes to the particles removed from the system due to recombination and leakage. We adopt a over-relaxation type of correction technique, the over-correction is used to speed up the over-all convergence rate. As defined before, if $B F>1$, the system has more particles introduced 
into than eliminated, but if $\mathrm{BF}<1$, the opposite is true, The over-correction factor is proportional to $(B F)^{k}$, i.e., to the kth power of the balance factor. The value of $k$ initially chosen was $k=2$, but an optimum value can be obtained by trial and error. 
CHAPTER III

VARIANCE REDUCTION TECHNIQUES

\section{A. General Background}

The Monte Carlo method (or method of statistical

trials) consists of solving various problems of computational mathematics by the construction of some random process for each such problem, with the parameters of the process set equal to the required quantities of the problem. These quantities are then approximated by observations of the random process and the computation of its statistical characteristics, which are approximately equal to required parameters [from Schreider (15)]. Every Monte Carlo computation that leads to quantitative results may be regarded as estimating the value of a multiple integral. Suppose we have $M$ random numbers in the computation system. The results will be a vector valued function $R\left(\xi_{1}, \xi_{2}, \ldots \varepsilon_{M}\right)$ involving the sequence of random numbers $\xi_{1}, \xi_{2}, \ldots \xi_{\mathrm{M}}$. This is an unbiased estimator of $\int_{0}^{1} \ldots \int_{0}^{1} R\left(x_{1}, x_{2}, \ldots x_{M}\right)$ $d x_{1} \ldots d x_{M}$. For the sake of simplicity, we take the onedimensional integral as a standard example. Let

$$
\theta=\int_{0}^{1} f(x) d x
$$

where $f \in L^{2}(0,1)$ or $\int_{0}^{1}[f(x)]^{2} d x$ exists.

Define the relative efficiency of two Monte Carlo methods as follows. Let the methods call for $n_{1}$ and $n_{2}$ units of computing time, respectively, and let the resulting 
estimates of $\theta$ have variances $\sigma_{1}^{2}$ and $\sigma_{2}^{2}$. Then the efficiency of method 2 relative to method 1 is defined as

$$
\frac{n_{1} \cdot \sigma_{1}^{2}}{n_{2} \cdot \sigma_{2}^{2}}
$$

Equation 35 is the product of two terms, the variance ratio $\sigma_{1}^{2} / \sigma_{2}^{2}$ and the labor ratio $n_{1 / n_{2}}$. If $\eta_{1}, \eta_{2}, \ldots \eta_{N}$ are independent random numbers with uniform distribution between 0,1 or $\eta_{i} \in(0,1)$; then the quantities $f_{i}=f\left(\eta_{i}\right)$ are the independent random variates with expectation $\theta$. Therefore

$$
\bar{f}=\frac{1}{N} \sum_{i=1}^{N} f_{i}
$$

is an unbiased estimator of $\theta$, and its variance is

$$
\frac{1}{n} \int_{0}^{l}(f(x)-\theta)^{2} d x=\frac{\sigma^{2}}{n}
$$

Accordingly the quantity $\bar{f}$, which has been determined by observation of the random process, is approximately equal to the required quantity $\theta$ (or in other words, it is an unbiased estimator of $\theta$ ) with a probability which can be made as close as required to unity if a large number of trials is practical. We refer to the estimator $\bar{f}$ as the crude Monte Carlo estimator of $\theta$ (commonly referred to as Monte Carlo estimation).

Let us introduce another even less efficient method, namely hit or miss Monte Carlo. Suppose that $0 \leq f(x) \leq 1$ 
when $0 \leq x \leq 1$. We may draw the curve $y=f(x)$ in the unit square $0 \leq x, y \leq 1$, and $\theta$ is the proportion of the area of the square beneath the curve. Stating this formally, we write

$$
f(x)=\int_{0}^{1} g(x, y) d y
$$

where $g(x, y)=0$ if $f(x)<y$

$$
=1 \text { if } f(x) \geq y
$$

Then

$$
A=\int_{0}^{1} \int_{0}^{1} g(x, y) d x d y
$$

The estimator

$$
\bar{g}=\frac{1}{n} \sum_{i=1}^{n} g\left(\xi_{2 i-1}, \xi_{2 i}\right)=\frac{n *}{n}
$$

$n *$ is the number of occasions where $f\left(\xi_{2 i-1}\right) \geq \xi_{2 i}$

In other words, we take $\mathrm{n}$ trials at random in the unit square, and count the proportion of them which lie below the curve $y=f(x)$.

Historically, a hit or miss method was first propounded in the explanation of Monte Carlo techniques. It is the easiest to understand but one of the least efficient techniques. The rate of convergence and the variance (statistical error) is in general proportional to $1 / \sqrt{\mathrm{N}}$ where $\mathrm{N}$ is the number of samples. It is obviously impractical to gain a significant improvement of accuracy by merely increasing $N$. Therefore more efficient techniques for reducing the variance and/or increasing the efficiency 
are very important. Monte Carlo experimentalists need. wide experience and background in both the mathematics and the physics of the problem, and they have to exercise considerable ingenuity in distorting and modifying problems in the pursuit of variance reduction techniques. Statistical and inferential procedures are also important in order to extract the most reliable conclusions from the observational data.

The basic requirement of the Monte Carlo calculations is to establish how random numbers may be used to sample a function that describes, in a probabilistic fashion, a physical event. The procedure by which this is done follows from the fundamental principle of Monte Carlo (16), which may be stated as follows. If $p(x) d x$ is the probability of $x$ lying between $x$ and $x+d x$, with $a \leq x \leq b$

$$
\int_{a}^{b} p(x) d x=1
$$

Then $\quad r=P(x)=\int_{a}^{x} p(y) d y$

determines $\mathrm{x}$ uniquely as a function of the random number $\mathrm{r}$ which is uniformly distributed on range $(0,1)$. The quantities $p(x), P(x)$ are the probability density and the probability distribution functions, respectively.

With the development of large computers, the use of the Monte Carlo method in a wide range of problems has 
increased rapidly. In particular, the method is applied to various physical problems such as plasmas (15), radiative transfer problems (21), neutron transport and nuclear reacter physics (29), etc.

The random walk type simulation can be briefly described in the following manner. One first introduces the basic physics of the problem into the computer in a probabilistic fashion. A system of coordinates and boundaries are defined and then, as a computer experiment, particles are released from the source. These particles are traced as they diffuse through a prescribed medium following the probabilistic interaction laws. The particles are followed until they escape from the medium or are absorbed or are converted to the thermal field.

The parametexs pertinent to the evaluation of the desired quantities are recorded. One continues processing additional particles until adequate statistical estimates of the quantities of interest have been obtained.

When doing a Monte Carlo problem one focusses attention on three main topics. They are:

1. The development of the analogy for the probability processes (simulation).

2. The generation of sample values of the random variables on a given computing machine.

3. The design and use of variance reduction techniques. 
The variance reduction methods are often strongly dependent on the probabilistic model, thus the greatest gains are often made by exploiting specific details of the problem, rather than by routine application of general principles.

\section{B. Importance Sampling}

The general idea of importance sampling is to draw samples from a distribution other than the one suggested by the problem. Then an appropriate weighting factor is introduced to correct the biasing caused by changing the original distribution. If done correctly the final results are essentially unbiased. The object is to concentrate the distribution of sample points in the parts of the region that are most "important" instead of spreading them out evenly. Thus, the probability of sampling from an interesting region is increased; the probability of sampling from unimportant or less interesting region is correspondingly decreased. Stating this formally, we have

$$
\theta=\int_{0}^{1} f(x) d x=\int_{0}^{1} \frac{f(x)}{g(x)} g(x) d x=\int_{0}^{1} \frac{f(x)}{g(x)} d G(x)
$$

for any functions $g$ and $G$ satisfying

$$
G(x)=\int_{0}^{x} g(y) d y
$$

where $g$ must be a positive valued function. such that 


$$
G(1)=\int_{0}^{1} g(y) d y
$$

if $\eta$ is a random number sampled from the distribution $G$, then $f(\eta) / g(\eta)$ has the expectation $A$ and variance

$$
\sigma_{f / g}^{2}=\int_{0}^{1}\left(\frac{f(x)}{g(x)}-\theta\right)^{2} d G(x)
$$

We notice that if $g(x)=c f(x)$, or if $g$ is proportional to $f$ and if $c=\frac{1}{\theta}$ then $\sigma_{f / g}^{2}=0$. This perfect situation does not exist, since we do not know $\theta$. We always obtain an unbiased estimate for positive function $\mathrm{g}$. Our object is to select $g$ to reduce the standard error of our estimate.

Because the estimate is the average of observed values of $f / g$, we choose $g$ such that $f / g$ is as constant as possible in order to achieve a small sampling variance. While we intend $g$ to mimic $f$, we generally restrict our choice of $g$ to functions simple enough to allow analytic integration.

For the present plasma problem, the importance sampling technique is developed as follows: during the particle's (electron's) random walk, it suffers a large number of collisions with gas atoms, and these collisions may be elastic or inelastic or of other kinds. The probabilities of their occurrence depend on their cross section $\Sigma_{i}$. We have

$$
\Sigma_{t}=\Sigma_{1}+\Sigma_{2}+\cdots \Sigma_{M}
$$


where $\Sigma_{i}$ : the cross section for $i$ th process

$\Sigma_{t}$ : total cross section or the summations of the cross sections of all the possible processes that the particle may experience.

Dividing both sides of equation 47 by $\Sigma_{t}$, we obtain:

$$
1=p_{1}+p_{2}+\cdots \cdot p_{M}
$$

where $\mathrm{P}_{i}=\Sigma_{i} / \Sigma_{t}$ the probability of $i$ th process occurring as the particle experiences a collision. If we introduce weights $w_{i}$ into equation 47 we obtain

$$
\Sigma_{t}^{\prime}=w_{1} \cdot \Sigma_{1}+w_{2} \cdot \Sigma_{2}+\cdots \cdot w_{M} \cdot \Sigma_{M}
$$

Similarly we have

$$
I=p_{1}^{\prime}+p_{2}^{\prime}+\cdots \cdot p_{M}^{\prime}
$$

After the application of the weights, we put more weights on the interesting or more important events. Then their artificial probabilities are greater, therefore we can get better estimates of the important parameters. Coincidently, such a linear weighting scheme can only be applied to the elastic colitision process in our system. The other collision processes involve appreciable changes in the particle's energy (catastrophic collision). Also cross sections are energy dependent, these nonlinear effects prevent the application of above mentioned weighting scheme to inelastic collision processes. By looking at the cross section values, in the low energy region, the elastic cross section is by 
far the largest by order of magnitude. Others have shown that the elastic process does not strongly influence the estimated parameters that we are interested in $(1,2)$. As an electron makes an elastic collision, it loses only a small amount of energy while making a small angle deflection. In fact, most other workers simply neglect elastic loses if an electric field is present (1, 2, 10). In our case, with $E_{f}=0$, elastic effects must be included. In the low energy region ( $\approx 0 \mathrm{eV}$ to $20 \mathrm{eV})$, the energy of a majority of the particles lies below $I \mathrm{eV}$ and over $90 \%$ collisions are elastic collisions, and only a few collisions are inelastic.

The collision process subroutines are a time-consuming part of the simulation calculations. If we can supress: the elastic processes by a factor of $k_{e l}$ the chances of all other processes are increased by approximately the same factor. Therefore we convert more computing time to processes which contribute more strongly to the quantities of interest. The following formulation provides the theoretical support for this. In lieu of equations 47-50, the $p_{i}$ are the original probabilities (undistorted probabilities) while the $p_{i}^{\prime}$ are the weighted probabilities. Then the correction factors are introduced as follows; at energy $E$, we have

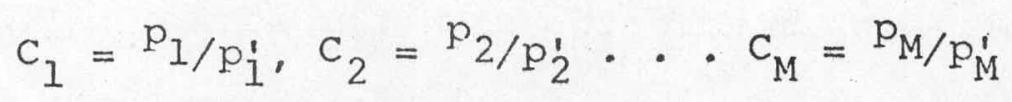


or $\quad c_{i}=p_{i / p_{i}}=\frac{\Sigma_{i} \cdot \Sigma_{t}}{\sum_{t} \cdot \Sigma_{i} w_{i}}=\frac{\sum_{t}}{\sum_{t}} \frac{1}{w_{i}}$

If we let $w_{1}=\frac{1}{k_{e l}}, w_{2}=w_{3}=\ldots . w_{M}=1$

then from (51) we have

$$
c_{1}=\frac{\Sigma_{1} \cdot \Sigma_{t}}{\Sigma_{t} \Sigma_{1} \cdot w_{1}}=\frac{\Sigma_{t}}{\Sigma_{t}} \frac{1}{w_{1}}, c_{i}=\frac{\Sigma_{t}^{i}}{\Sigma_{t}}=1, \quad i \neq 1
$$

let $\Sigma_{I}=\Sigma_{\text {el }}$ namely we introduce weight on elastic cross section only. Since $\Sigma_{e l}(E) \gg \Sigma_{i}(E), i>1, \forall_{i}$ then

$$
\frac{\Sigma_{t}^{\prime}(E)}{\Sigma_{t}(E)} \approx \frac{\Sigma_{e l}^{\prime}(E)}{\Sigma_{e l}(E)} \quad w_{1}=\frac{1}{k_{e l}}
$$

and $\quad c_{1} \simeq w_{1} \frac{1}{w_{1}}=1, c_{i} \simeq \frac{1}{k_{e l}} \quad(i \neq 1)$

Equation 53 holds for low energy region, which is approximately independent of energy.

\section{Russjan Roulette and Splitting}

In general, the sampling is done such that we examine the sample and classify it as being in some sense "interesting" or "uninteresting". We are willing to spend more than average amount of work on the interesting samples and on the contrary, we want to spend less effort on the uninteresting ones. This can be done by splitting the interesting samples into independent branches, thus resulting in more of them, and by killing off some of the uninteresting ones. The first process is splitting and the second Russian Roulette. 
The "killing off" is done by a supplementary game of chance. If this game is lost, the sample is killed; if it is won, the sample is counted with an extra weight to make up for the fact that some other samples have been "killed". The game has a certain similarity to the Russian game of chance played with revolvers - whence the name.

For the present plasma system we divide the whole energy spectrum into two regions, the low energy region I ( $0 \mathrm{eV}$ to $\mathrm{EDI} \mathrm{eV}$ ) and the high energy region II (EDI to $\mathrm{E}_{0}$ ). Here $\mathrm{E}_{0}$ is energy of source particles, which is the highest energy the particle can have. EDI is the energy region dividing line, which can be chosen as any valuebetween $5 \mathrm{eV}$ and $100 \mathrm{eV}$ depending on theinitial guessed energy distribution. In region I, the number density is high, and the elastic process is the dominant process. As we mentioned earlier, the elastic process does not contribute much, so Region I is classified as uninteresting. Each particle in this region (particles with energy less than EDI) is assigned a weight $k l(k I \geq 1)$. In other words, each particle plays the role of $\mathrm{kl}$ particles.

In region II, classified as interesting region, each particle carries a weight $w_{2}\left(w_{2}=1 / k_{2}, w_{2} \leq 1\right)$. Namely, each particle in this region is split into $\mathrm{k}_{2}$ particles with weight $\mathrm{w}_{2}$.

As particles cross over to other regions, care must be taken to account for killing off and splitting effects. 
For instance, a particle in region I may acquire enough energy, either from electric field or by superelastic collision with the excited atoms, to cross over to region II. Then we should generate $k_{3}$ particles $\left(k_{3}=k_{1} \cdot k_{2}\right)$ in region II with the same energy.

Particles from the high-energy region may lose part of their energy by inelastic collision such that they cross over to the low-energy region. Then killing off should be effective. For every $k_{3}$ such particles that cross over from region II to region I, only one particle survives. The values of $k_{1}$ and $k_{2}$ can be chosen according to the total number of particles in the system to be processed, the source rate, and related accuracy requirements. They are input parameters.

\section{Initial Guess}

Monte Carlo methods comprise that branch of experimental mathematics which is concerned with experiments on random numbers. The user, like the experimental physicist needs theory and knowledge to give structure and purpose to his experiments, and as experimental work provides growing insight into the nature of a problem and suggest appropriate theory. Good Monte Carlo practice should keep this relationship as a general maxim.

The basic procedure of the Monte Carlo method is the manipulation of random numbers, but these numbers should not 
be employed prodigally. Each random number is a potential source of added uncertainty in the final result. Thus it will usually pay to scrutinize each part of a Monte Carlo experiment to see whether that part can not be replaced by an exact theoretical analysis contributing no uncertainty. In other words, exact analysis should replace Monte Carlo sampling wherever possible. Sometimes reliable intuition would aid in increasing the efficiency of Monte Carlo calculations. For the plasma system we are simulating, the straight-forward approach would be to follow $N$ source particles on their life time history, until they lose energy sufficient by collisions to reach a thermal equilibrium. over 50 inelastic collisions generally occur, and the probability (inelastic cross sections) for inelastic processes is low. Consequently, the particles travel a relatively large distance which consumes a good amount of time. Thus a large number of time cycles would be required before the system reaches steady state. To avoid this, we use our intuition, to start off with a guessed initial distribution. The electron energy distribution $f(E)$ (or electron number density distribution) is a function of the electron's energy E. Initial particle data is generated using this distribution, and this can save considerable irrelevant computations. This idea is simple, yet very useful and effective. Without an initial distribution, the problem would require hundreds of time cycles to 
converge. However, with a reasonable guess, good results have been obtained in as little as tens of time cycles. of course, the better the initial guess, the faster the convergence.

\section{E. Antithetic Variates}

When we estimate an unknown parameter $\theta$ by means of an estimator $t$, we may seek another estimator $t$ ' having the same (unknown) expectation $\theta$ as $t$ but a strong negative correlation with $t$. Then $1 / 2\left(t+t^{\prime}\right)$ will be an unbiased estimator of $\theta$, and its variance is

$$
\operatorname{Var}\left[\frac{1}{2}\left(t+t^{\prime}\right)\right]=\frac{1}{4} \operatorname{var}(t)+\frac{1}{4} \operatorname{var}\left(t^{\prime}\right)+\frac{1}{2} \operatorname{cov}\left(t, t^{\prime}\right)
$$

where $\operatorname{cov}\left(t, t^{\prime}\right)$ is negative, and

$$
\begin{aligned}
& \operatorname{var}(t)=\varepsilon\left[(t-u)^{2}\right] \\
& \operatorname{cov}\left(t, t^{\prime}\right)=\varepsilon\left[(t-u) \cdot\left(t^{\prime}-u^{\prime}\right)\right]
\end{aligned}
$$

$u, u$ ' are the mean of $t, t$ ' respectively, and $\varepsilon(x)$ is the expectation of the random variable $\mathrm{x}$.

It is possible to make var(ttt') smaller than var(t) by suitably selection t'. For example, l-r is uniformly distributed whenever $r$ is, and if $f(r)$ is the unbiased estimator of $\theta$, so is $f(l-r)$. When $f$ is a monotone function, $f(x)$ and $f(1-r)$ will be negatively correlated. Thus we could take

$$
\frac{1}{2}\left(t+t^{\prime}\right)=\frac{1}{2} t(r)+\frac{1}{2} f(1-r)
$$


as an estimator of $\theta$.

The name and idea of antithetic variates was first introduced by Hammersley and Morton (13, 14) during attempts to improve Buffon's needle experiment for estimating the value of $\pi(3.1415926)$.

The main idea of antithetic variates is based on the underlining theorem [from Hammersley and Morton (12)] which can be stated informally as follows: "Whenever we have an estimator consisting of a sum of random variables, it is possible to arrange for a strict functional dependence between them, such that the estimator remains unbiased, while its variance comes arbitrarily close to the smallest that can be attained with these variables". As the name implies, antithetic variates are the set of estimators which mutually compensate each other's variations. Essentially, we rearrange the random variables by permuting finite subintervals in order to make the sum of the rearranged functions as nearly constant as possible. From the practical viewpoint, the mathematical conditions imposed by antithetic variates to the Monte Carlo calculation are quite loose and flexible. It is relatively easy to find a negatively correlated unbiased estimator to produce an efficient variance reduction scheme.

A system of antithetic variates is obtained by simple transformations based on a stratification of the interval (12). The transformations are constructed in such a way 
that the efficiency of the method will increase as a higher power of $M$ (the number of antithetic variates taken). The transformations considered are linear combinations of the values of the function $f$ at a number of points. Thus a correlated stratified sampling technique is developed, and its efficiency generally exceeds that of crude sampling by a factor $\mathrm{M}^{3}$.

The following useful transformations are obtained in this manner:

$$
\begin{aligned}
t_{1} & =\frac{1}{n} \sum_{i=1}^{n}\left\{\alpha f\left(\alpha r_{i}\right)+(1-\alpha) f\left[\alpha+(1-\alpha) r_{i}\right]\right\} \\
& =\frac{1}{n} \sum_{i=1}^{n} G_{\alpha} f\left(r_{i}\right) \\
t_{2} & =\frac{1}{n} \sum_{i=1}^{n}\left\{\alpha f\left(\alpha r_{i}\right)+(1-\alpha) f\left[1-(1-\alpha) r_{i}\right]\right\} \\
& =\frac{1}{n} \sum_{i=1}^{n} \mathcal{L}_{\alpha} f\left(r_{i}\right)
\end{aligned}
$$

The value of $\alpha$ is between 0 and 1 , the variance $\operatorname{var}\left(t_{j}\right)$ has a minimum at some value of $\alpha$. An adequate rule of thumb is to choose $\alpha$ by finding the root of

$$
f(\alpha)=(1-\alpha) f(1)+\alpha f(0)
$$

Another useful transformation is

$$
t_{3}=\frac{1}{m} \sum_{j=0}^{m} f\left(\frac{r+j}{m}\right)=U_{m} x(r)
$$


In general, the variance of crude Monte Carlo is $\operatorname{var}(t)=0\left(\mathrm{~m}^{-1}\right)$ while the variance of $t_{3}$ is $\operatorname{var}\left(t_{3}\right)=$ $O\left(m^{-2 M}\right),(M>1)$. The simplest form of antithetic transformations have proved quite successful in neutron transport calculations.

The general notion and a simple form of antithetic variates are applied in our present calculations. High efficiency gain can be obtained easily if it is used sparingly and at judicious points in the computation. We have used the random number $r_{i}\left(r_{i} \in(0,1)\right)$ most of the time for playing the law of chances, and $1-r_{i}$ is another random number of the same kind which is the image of $r_{i}$. The two are used together in good many places where repeated use of $r_{i}$ is required. This serves the purpose of two antithetic variates and cuts the labor in half (same random number $r_{i}$ being used twice). Equations 55 and 59 have been used in the initial data preparation program and source routine where initial particles states $\left(E_{i}, u_{i}, z_{i}, w t_{i}\right)$, $E_{i}$ are generated according to input initial distribution. For instance:

$$
\begin{aligned}
& u_{i}=2 r_{i}-1, u_{i+1}=2\left(1-r_{i}\right)-1 \\
& z_{i}=r_{i} \cdot d, z_{i+1}=\left(1-r_{i}\right) \cdot d \\
& E_{i}=E_{k}+r_{i} \cdot \Delta E_{k}, E_{i+1}=E_{k}+\left(1-r_{i}\right) \cdot \Delta E_{k} \\
& E_{i}=E_{0} \text { for source particles. }
\end{aligned}
$$

Here $k$ is an energy index, or $k$ th energy interval. Wherever $r_{i}$ appears, $r_{i}$ and $l-r_{i}$ are used interchangeably. 
Thus we are generating source particles from diametrically opposite directions, or from symmetric locations. F. Double History (Double processing) Technique In the particle tracing routine, using the general idea of antithetic variate technique, we have adopted a "so-called" double history processing technique which is described as follows;

a) In the course of tracing the particle's history, in each time cycle, we use the transformed random number $\lambda \cdot|\ln |$ to decide the distance to collision $d_{\operatorname{col} I}$ and go on until the particle reaches census time. However, we process the same particle twice under the identical initial condition except that we use $\lambda \cdot|\ln (1-x)|$ as the

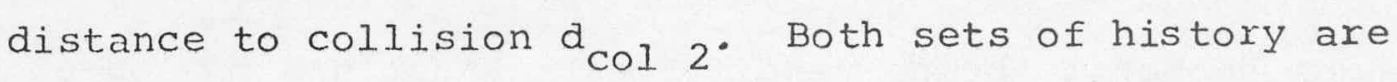
ta lied but only one set of particle data is'stored. This way each particle is processed twice independently but correlated in such a way that they compensate each other's variation (variance).

b) Another alternative for double history processing is, to play a dual direction random walk instead of dual collision distances. We process the same particle twice in such a way it looks like there are two identical particles emerging into the system in two diametrically opposite directions. Again both histories are tallied, but only the original particle's data is stored. 
The two versions of the double history processing technique increase the labor (computation time) by a factor of 1.2 to 1.5 . The efficiency gain is difficult to specify. At least it is certain that the gain is more than double the total number of the particles in the system.

The purpose of dual processing is to compensate each particle's variation and minimize the variance (statistical errors). One set of particle data in dual processing is thrown away, while the other set is stored just like normal processing. Which one of the two resulting sets is saved is completely random. 


\section{CHAPTER IV}

\section{MONTE CARLO SIMULATION CODE}

\section{A. Introduction}

This chapter plesents the complete Monte Carlo simulation program, including an input, output, main flow chart, and the background theory and techniques incorporated. This program implements the condensed history approach that was described in Chapter II together with various variance reduction techniques described in Chapter III.

The basic Monte Carlo calculation can be described most easily by reference to the simplified flow chart shown in Figure 4. By means of this flow chart we shall follow the particle tracing process, making relatively brief comments on the various steps implied by each box, on each routine, and on the main program variables. The program is written in FORTRAN IV and is designed to be run on IBM $360 / 75$ under the HASP-MVT system.

\section{B. Calculational. Procedure and Program Description}

To obtain an overall view of the calculations, we note that there are three basic steps involved in the simulation process. The first is the initialization of all parameter values, i.e., prepare the particle data and predict the unknown dominate parameter values. The second is the main loop of particle tracing which generates census information. The third uses the census information and takes tallies to estimate the histograms (electron energy or number density 


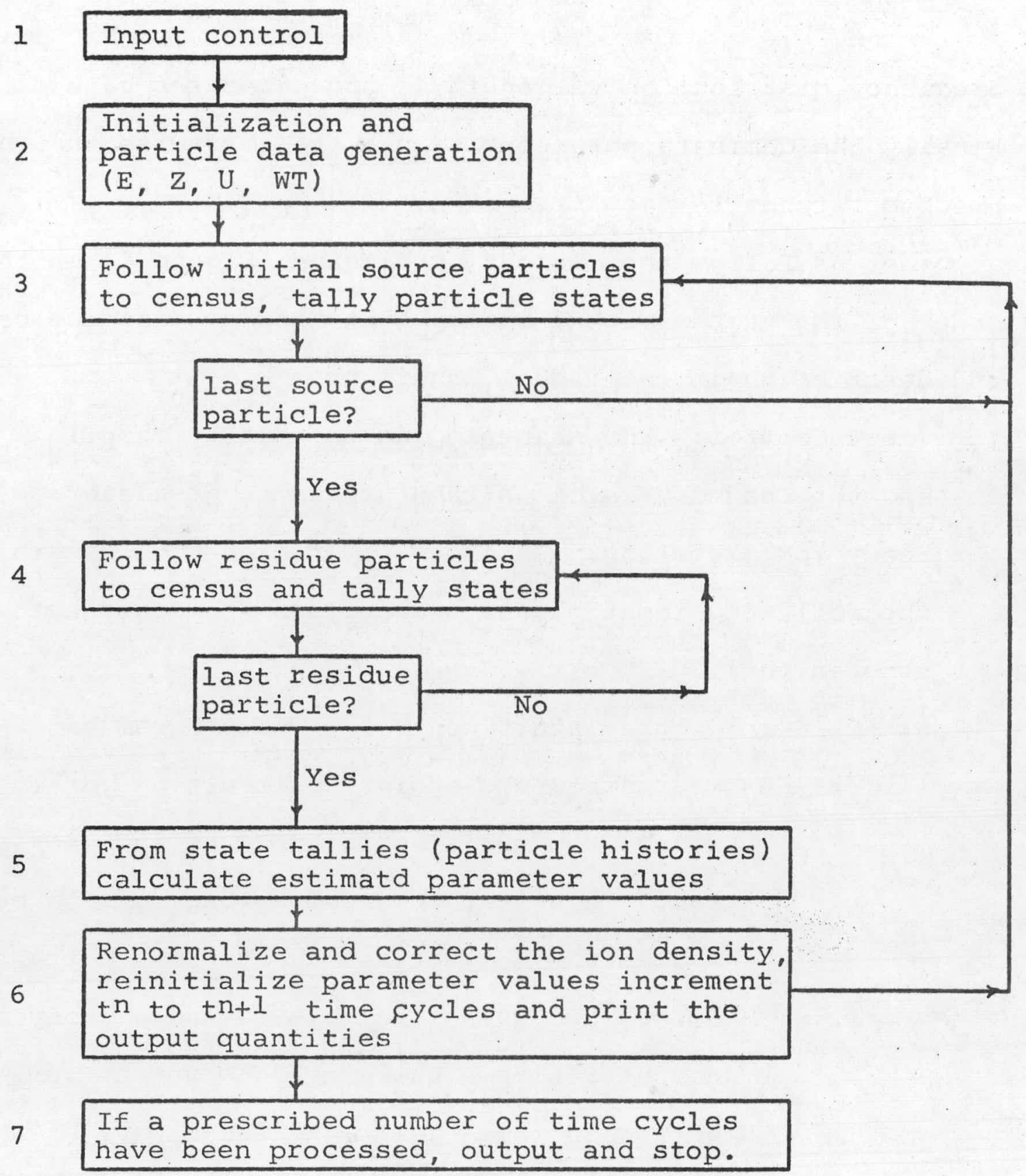

Figure 4. Flow chart of the Monte Carlo simulation code. 
frequency distribution vs. energy), and parameter values. Finally the dominate parameter va ues are corrected and the program returns to the first step.

Let us follow the logic flow diagram (Figure 4) in the order of the number marked on the left hand side of the boxes, and describe their individual functions.

1. Input Control: The main program is usually compiled and the loaded version is then stored on disk storage, ready for execution. In order to offer a wide flexibility, the following input parameters are used to control the program runs.

a) NDISK: program start control. The program can start from the beginiing (cycle 0 ), or it can start from the stopping point of the previous run. This feature enables long runs to be broken up into several short runs. It also offers debugging flexibility and economy of computer time. While a long run may run up to 150 time cycles, we can always break it up into multiple numbers of fives and tens cycles. Thus we can adjust other parameter values on a cut and try basis without wasting much computing time. The input parameter for this control purpose is NDISK: input 0 for storing from very beginning; input 1 for starting from the latest tenth cycle of last 
run; input 2 for starting from the latest fifth cycle.

b) K1, K2, and EDI: Regional weights and dividing energy. The Russian Roulette and splitting variance reduction techniques use three input parameters to control the amount of killing off and/or splitting of particles. This also determines where to divide energy regions for the Russian Roulette and splitting, assuming that the option to use Roulette is selected. The input parameters are Kl: the weight of particles in region $I$ $(K I \geq 1), K 2:$ the inverse of the weight w2 of particles in region II where $\mathrm{w} 2=1 / \mathrm{K} 2$ ( $\mathrm{K} 2 \geq 1$ ), EDI: the energy dividing line,

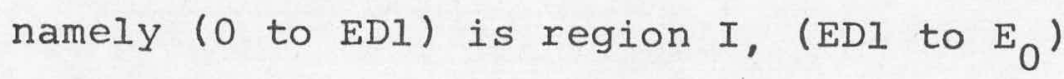
region II where the energies are in electron volts.

c) NTC: The total number of timecycles to be run. DTC: The input parameter to control the length of census time $\Delta t$ of the time cycles. In terms of distance travelled by the particles, $\Delta t$ is equal to a fraction or multiple of one mean free path, corresponding to average energy of particles in the system. 
d) NTP: The total number of particles that actually exist in the simulation system.

NTW: The artificial* total number of particles in the system during Russian Roulette and splitting games.

NS: The number of source particles injected into the system during each time cycle.

e) KELC: The input parameter that controls the importance sampling option of the program, i.e., the multiplying factor used to suppresis the elastic cross section (reduces the probability of elastic process by a factor KELC).

f) EDP: The input value of the ratio of the electric field strength to the pressure. PDPO: The input value for the reduced pressure of the system $\left(\mathrm{p} / \mathrm{p}_{0}\right.$ where $\mathrm{p}_{0}=1$ torr $)$.

g) NIP: The input parameter used to control double processing: NIP $=2$ for double processing, NIP $=1$ for single processing. IUSN : $\quad$ IUSN $=1$ for dual-collision type double processing IUSN $=-1$ for dual-direction type double processing

* The total number modified by scaling and weighting factors. 
h) D: The input value of the width of the slab (diameter of the cylinder) which defines the external boundaries of the medium. JZ: The number of zones the slab is divided into, or the number of internal zones.

i) NCUM: Inp $t$ control to give cumulative results (time average) over the time cycles or a cumulative average over NCUM time cycles. NAVP: Input constant to control the power of $\Delta t$, (or $\Delta D$ ) namely $\Delta t_{i} * * N A V P$ is the weight of the cycle to be used for cumulative results. These two parameters are used to implement antithetic variates techniques along the time axis.

j) EO: The energy of source particles.

SR: The rate of the source particles entering the system.

k) FID: An array that contains the initial guess distribution (number density vs. energy).

1) NUSC: An input parameter used to control the upscattering elastic collision process; NUSC = I: with upsacttering, NUSC $=0$ : without upscattering.

2. Data preparation: The initialization is entered only once during the calculation, where the time cycle is 0 (or at time $t_{0}$ ). After the input phase, all the pertinent 
parameters are set to their proper permanent or temporary values. Before entering the next phase to trace the particle histories, several dominate parameter values must be predicted. The data preparation routine is called in to generate particle data $\left(E_{i}:\right.$ energy, $u_{i}$ : direction of travel, $\mathrm{Z}_{i}$ : position, $W t_{i}$ : weight) based on the guess for the initial distribution (generate machine particles). Then the average energy of the system is calculated and is used to predict the census time ( $\triangle t$ or equivalently DCEN) as well as the number of ions $\left(N^{+}\right)$.

3. Particle tracing: With the point of origin, direction of propagation, and energy (velocity) known for the initial source particles, the next phase follows the particles through a series of stochastic interactions until the census time is reached, and the particle data and parameters values are tallied and updated. During the course of tracing the particle histories, all the relevant events are recorded and later on can be interrogated to provide the output quantities of interest. This section of the program is the central part of the main program. It contains the main loop for tracing all the particles in the system in a self consistent way. Most of the variance reduction techniques are incorporated here, including double processing, Russian Roulette, and splitting, as well as the various versions of 
importance sampling techniques.

4. Secondary particle tracing: The residue particles, i.e., particles generated by ionization collision process (the secondary electrons so produced) are treated in all respects like the regular source particles, except that their starting positions and time coordinates as well as directions are those associated with the particles that generated them. These residue particles may go further and produce other residue particles (secondary electrons) if their energy is high enough, thus forming a short production chain. The probability of such chain production is small however, since the average energy of the secondaries is quite low. The end of the main loop comes after all such residue particles are processed.

5. Data calculations: The details of the phase of the computations depend upon the specific goals and character of study. Our primary interest is the particle energy distribution (or number density vs. energy). This frequency distribution and other pertinent parameters are estimated (or calculated) using the stored data from various parameter counters in the main loop (tallied information) as well as tallied histories. The quantities of interest include local values (for the current time cycle only) and the cumulated values from several time cycles. After these calculations, the next phase is to prepare for the processing of new time 
cycle.

6. Normalization and printout: Up to this stage, all the output quantities have been calculated. Next the output information is printed out, and at the same time, if the cycle number is the multiple of. five, all the particle data and related parameter values are stored on the disk. Then the next run can be read off of the disk and the process can proceed from where we left off the last time. All the parameter values are again initialized, and new source particles are generated for the next time cycle. Particles that have escaped from the system or have been absorbed create holes in the particle data storage which are filled up by the new source particles and the residue particles produced during the time cycle. Before going on to the next cycle, the ion density is renormalized and corrected in a semiadaptive way according to a balance factor BF. The ion density distribution parameter $\mathrm{N}^{+}$is a dominate parameter since it affects several processes such as the rate of recombination and the rate of super-elastic collision (refer to Equations 27 and 28). It is corrected in such a way as to accelerate convergence, till the system reaches a balanced situation. Then increment time from $t^{n}$ to $t^{n+l}$. The output quantities are the following:

a) The kind of gas in the medium, source particle energy, pressure, slab thickness, and $\Delta t$ the time interval of this cycle. 
b) The number of time cycles, the time in seconds, the number of source particles generated during the cycle time, and the average energy of the particles in the system.

c) The number density distribution vs. energy, the single cycle distribution, the cumulated distribution, the number-density deviation, the averageenergy deviation, the average energy, and the energy interval vs, energy.

d) The number density and the average energy vs. $z$ (position zone).

e) The ion distribution $\left(\mathrm{N}_{+}\right)$, the number of ionizations (NIZN), the number of escaped particles (NES), the number of absorbed or recombined particles (NAS), the number of super-elastic collisions (NSE), the number of excitations to metastable levels (NMET), and the number of excitations to other levels (NEXC).

f) All the input control parameters as listed in section IV-I.

g) The electric field strength (EF), the source rate, the W-value, the average energy of escaped particles, and the average energy of absorbed particles.

h) The number check on the density disbributions.

All the above outputs include both current cycle values and the cumulated values over the past cycles. 
7. Final checks: The last stage is to check whether the prescribed number of time cycles have been processed, adequate statistics have been collected, and all the useful data have been stored on the disk. If so, the program stops.

Further programming details are provided in Appendix 


\section{CHAPTER V}

\section{RESULTS}

\section{A. Convergence and Reliability}

The present Monte Carlo Model is a predict-correct iterative scheme, with semiadaptive control ability. For such an iterative procedure, our main criteria for the evaluation and determination of convergence are based on the following inferences:

1. The balance factor (BF) as defined in Chapter II (Section II-B-3) signifies whether or not the system has reached steady state, i.e., converged to a final solution. At steady state, the value of BF should be approximately equal to unity. It may approach to unity in both directions, and due to semiadaptive control procedure used, it may oscillate around unity. Larger amplitude oscillations are observed at first, but they rapidly damp out.

2. The estimated values of the major parameters, e.g. the average energy and the W-value show a similar fluctuation, and then they converge to nearly constant values as steady state is approached.

3. The validity of the convergence has been checked using test problems where analytic results are available. A good comparison has been obtained, showing that the program and the model are working correctly, free of obscure errors. 


\section{B. Error Estimation and Analysis}

\section{Error Analysis}

1. As mentioned in early chapters, Monte Carlo simulation is equivalent to performing mathematical experiments, the results being based on observations of such experiments. From this analogy we can dexive the following procedures for the analysis of errors or accuracies of our calculations.

It is convenient to subdivide experimental errors into three broad types, namely: random errors, systematic errors, and blunders. In general, the experimental error is some additive function of all three, while blunders can hopefully be eliminated. We shall describe them in detail in following sections.

a. Random errors - such errors are of great concern in Monte Carlo type calculations. They generally involve statistical fluctuations or deviations, representing the difference between the singly measured value and the best value of a set of measurements, i.e., the difference between the arithmetic mean and the true mean. Sometimes such errors are referred to as elementary (or inherent) errors in the measurements. The commonly used measure of random errors is the variance. or standard deviation.

b. Systematic error - a systematic error tends to have the same algebraic sign, i.e. itis either an additive or subtractive quantity introduced in the measurement 
process. It is an unpleasant and insidious contribution which is not generally amenable to statistical treatment. Thus such errors seriously impair the reliability of the estimation. Typical examples include:

i) Incorrect assumptions or approximation in the representation of certain processes.

ii) Constructional faults and mistakes in the algorithms or subroutines.

iii) Inadequate regard of constancy of experimental conditions or inadequate sampling techniques (biased).

c. Blunders - these are outright mistakes which should be corrected by all means. Possible examples include:

i) Incorrect logic, misunderstanding of the problems.

ii) Errors in transcription of data.

iii) Mistakes in constants used.

iv) Confusion of units.

In any type of calculation, the systematic error as well as blunders should be removed. One way for detecting such errors is to compare the program against some known reliable solutions. Only the random errors are subject to reduction by the various treatments amenable to attack by variance reduction technqiues.

The precision in an estimated (mean) value is proportional to the reciprocal of the statistical error and is 
high if the statistical error is small, i.e. the accuracy is high if the net systematic error is small. Usually, but not necessarily, high accuracy implies small statistical errors as well.

As discussed in later sections, our model and program has been checked against several test problems, and good agreement has been obtained. Therefore, it can be assumed that any serious systematic errors have been removed and that the program is free of blunders. Thus, we shall concentrate on the analysis of random errors.

No thorough analysis of variance has been made. Due to the intricate way that the histories calculated during a given time cycle depend on previous time cycles, a meaningful variance estimate beyond the first time cycle seems to be out of the question. However as the system reaches steady state, the deviations or variations (statistical fluctuations) of the estimated parameters can be estimated by means of standard deviation (or variance) over subsequent time cycles. Namely, we use the following formulation:

$$
s_{i}=\left[\frac{\sum_{j=1}^{n}\left(f_{i j}-\bar{f}_{i}\right)^{2}}{n}\right]^{1 / 2} \neq i
$$

where $s_{i}$ : the standard deviation at the ith energy interval over $n$ consecutive time cycles.

$f_{i j}:$ the parameter value (density distribution function of ith energy interval, at jth time cycle.

$\bar{f}_{i .}:$ the average parameter value of ith interval over n time cycles. 
A heuristic approach was adopted to measure local deviations or statistical fluctuations at each time cycle. This provides a relative magnitude of deviation or dispersion which can be used to compare the various variance reduction techniques. The approach is described as follows:

The estimated major frequency distribution (the electron energy density distribution function in our case) is obtained in the form of histogram. The abscissa of the histogram is divided into so-called class intervals (energy intervals in our calculations). In each such interval an erect rectangle (block) of heigh $f_{i}$ and width $E_{i}$ is formed. This block-area type of distribution is the fitted frequency distribution curve. We devise a numerical description of local deviations by defining:

i) the location index of the center of the erected rectangles in the histogram as $i$ and with abscissa value $E_{i}$.

ii) the spread or dispersion around the center. The total number of particles in each class interval (energy interval) is proportional to the area of the rectangular flock ( $f_{i} \Delta E_{i}$ ), the particle's energy in this class interval falls in the range $\left(E_{i}-\Delta E_{i / 2}, E_{i}+\Delta E_{i / 2}\right)$ with overall average energy $\bar{E}_{i}=E_{i}$. Then the following relationship holds (refer to Figure 5): 


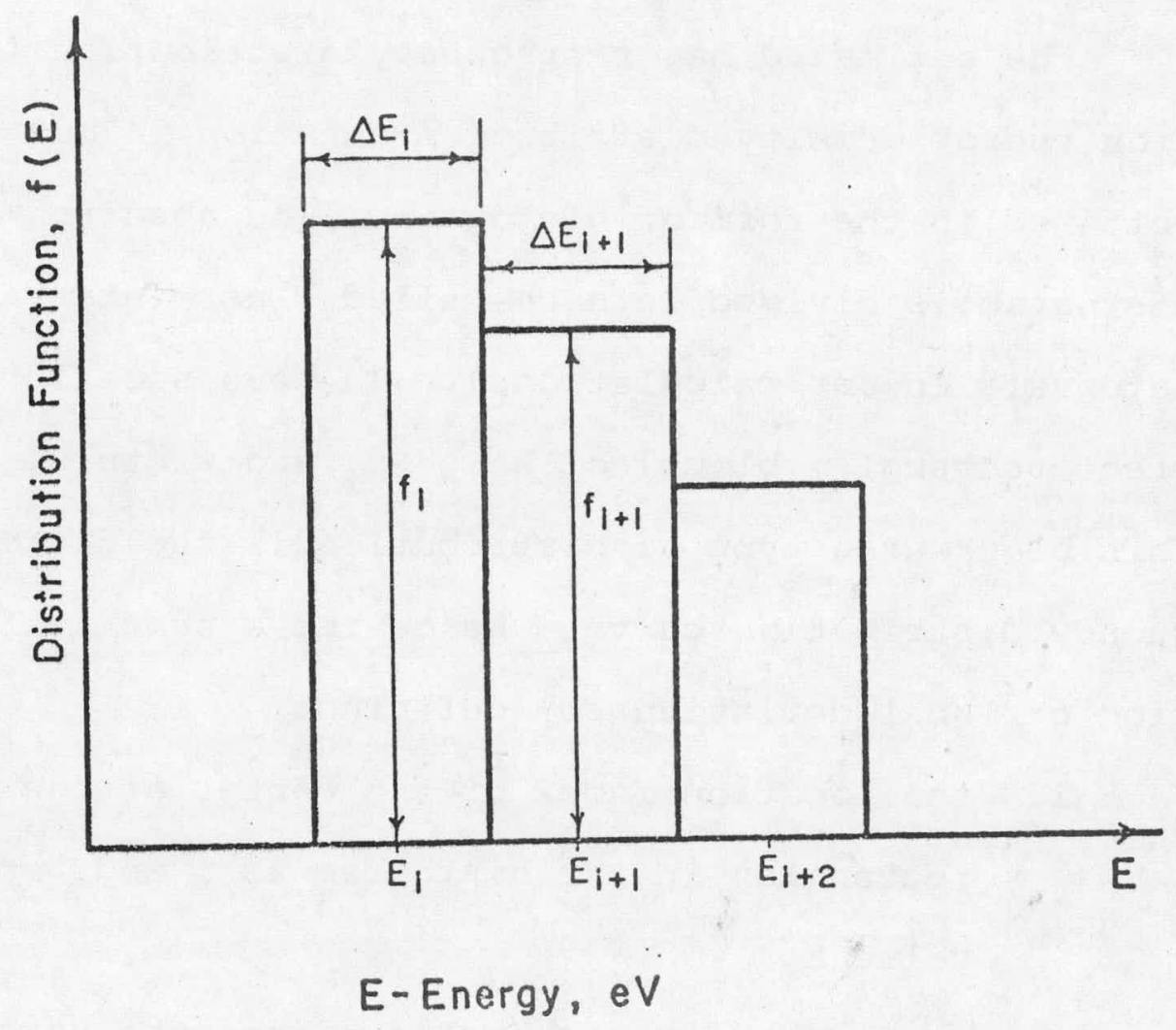

Figure 5. Rectangular block representation of the electron energy distribution histogram. 


$$
\frac{\Delta \bar{E}_{i}}{\Delta E_{i}}=\frac{\Delta f_{i}}{f_{i}}
$$

or $\Delta f_{i}=f_{i} \frac{\Delta \bar{E}_{i}}{\Delta E_{i}}$

where $\Delta \bar{E}_{i}$ is the deviation of the average energy $\bar{E}_{i}$ from $E_{i}$ or $\Delta \bar{E}_{i}=\bar{E}_{i}-E_{i}$. Also $\Delta f_{i}$ is the corresponding deviation of the frequency distribution at location index $i$.

Equation (62) provides a relative measure of the amplitude of the deviation of density distribution curve at energy $E_{i}$ which can be used for comparing the efficiency of different variance reduction techniques as well as provide an heuristic clue to the modification of the fitted density distribution curve.

\section{Data Filtering}

The random errors (statistical fluctuations) result in bumps and ridges in the estimated frequency distribution function. It may be possible to alleviate many of these distortions by means of data smoothing techniques (data. filtering).

Two types of data smoothing techniques commonly used in the processing of time series might be used here:

a. Moving averages - this technique operates by replacing each point of the frequency distribution function (height of the histogram at $E_{i} V_{i}$ ) at time cycle $t_{j}$ with an average value of several subsequent points in the time cycle series. Thus if $f_{i j}$ is the value 
of frequency function at ith energy interval and $j$ th time cycle, then $f_{i j}$ is replaced by

$$
\sum_{j=1}^{M} f_{i j} \cdot W_{j / M}
$$

where $w_{j}$, the weighting factor for the $j$ th time cycle, usually has the value of 1 or we can define it as a function of $\Delta t_{j}$. The value of $M$ is the number of successive points of the time cycles to be included in each average.

b. Parabolic filtering - this is similar to the moving averages technique, but $\mathrm{M}$ is selected as an odd number, the point to be replaced is located in the center of these $M$ points, and a parabola is fitted to these points by means of least squares. Thus it is replaced by the corresponding point $f_{i}$ on the derived parabola. The process is repeated by shifting the center point to the next one in the time cycle series.

The technique of moving averages (a) is employed in the program, because of it's simplicity and the fact that the parabolic smoothing technique is too sensitive to the changes in the distribution function. Note that this smoothing (filtering) technique only becomes effective as the simulated system reaches steady state. 


\section{Solutions of the Problem}

\section{Preliminary Checks}

In order to verify that the model is valid and correctly. working, and the computer program is free of bugs, blunders, and systematic errors, we solved a specific problem first reported by J. A. Smit (II) and later extended by Heylen and Lewis (10). Their solutions for the electron energy distribution were obtained analytically based on the Boltzmann transport equation for the special case characterized as follows:

i) electric field present

ii) no external sources

iii) infinite size medium of background noble gases. The comparisons are made for helium gas at $E_{f / p}=4,5$, $10 \mathrm{~V} ; \mathrm{Cm}^{-1}$. ( $\mathrm{E}_{\mathrm{f} / \mathrm{p}}$ : electric field strength to pressure ratio). The results are shown in Figures 6, 7 and 8 . Both smit and Heylen's results have taken into account all the collision processes including those due to elastic loss as well as inelastic losses. Heylen's results is more up-to-date, for low $\mathrm{E}_{\mathrm{f} / \mathrm{p}}$ values only $\mathrm{E}_{\mathrm{f} / \mathrm{p}}=5$ is given, which was chosen for the present comparison. In so far as possible, the same cross sections as Smit and Heylen and Lewis used were incorporated in the present calculations. The slight discrepancies apparant at the low-energy end is probably due to differences in treating the scattering process, and 


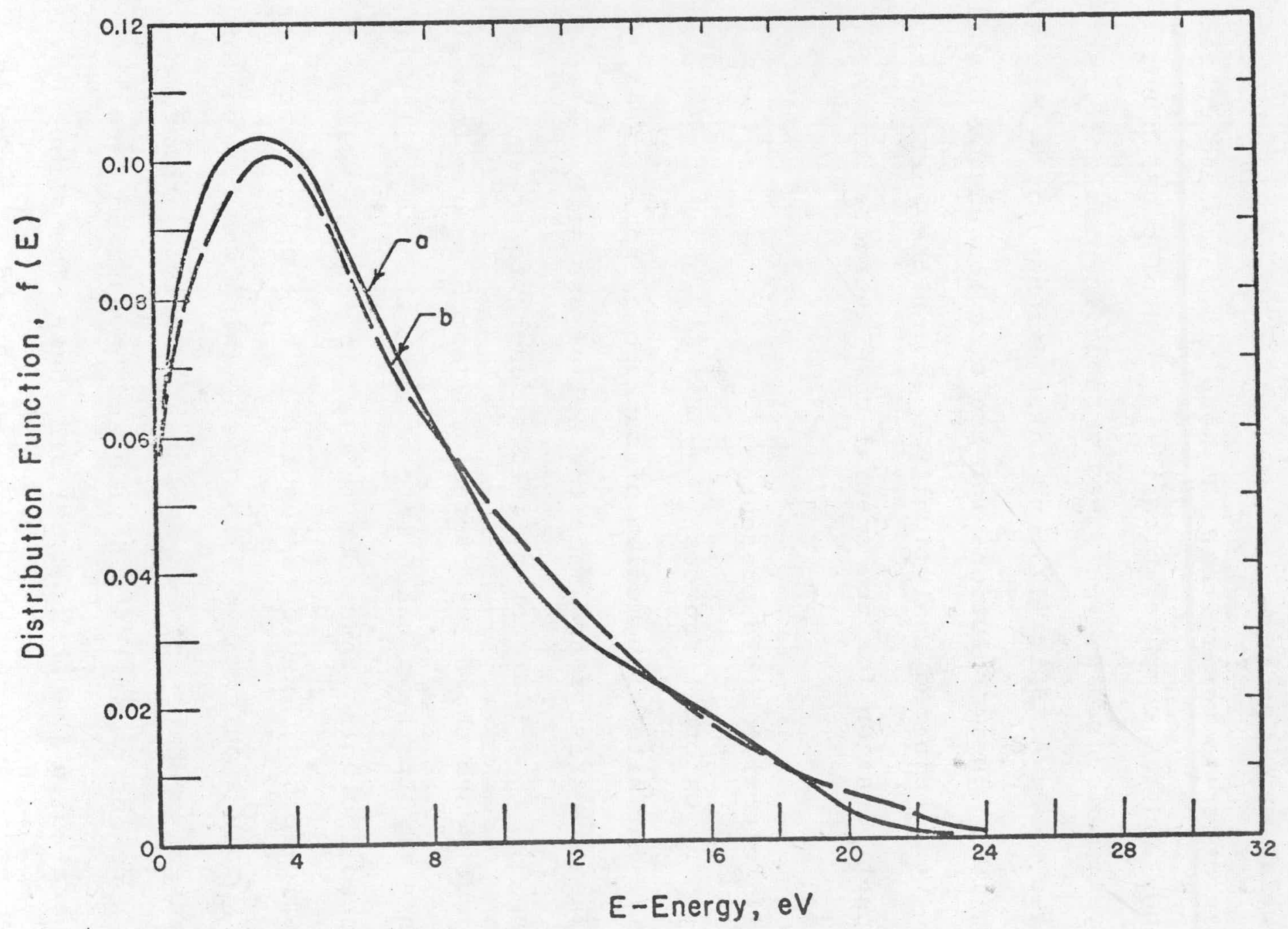

Figure 6. Comparison of the electron energy distribution for He at ${ }^{E_{f}} \mathrm{p}=4$ for a) Smit's calculation (1936) $(\bar{E}=6.4)$

b) present calculation $(\bar{E}=6.51)$ 


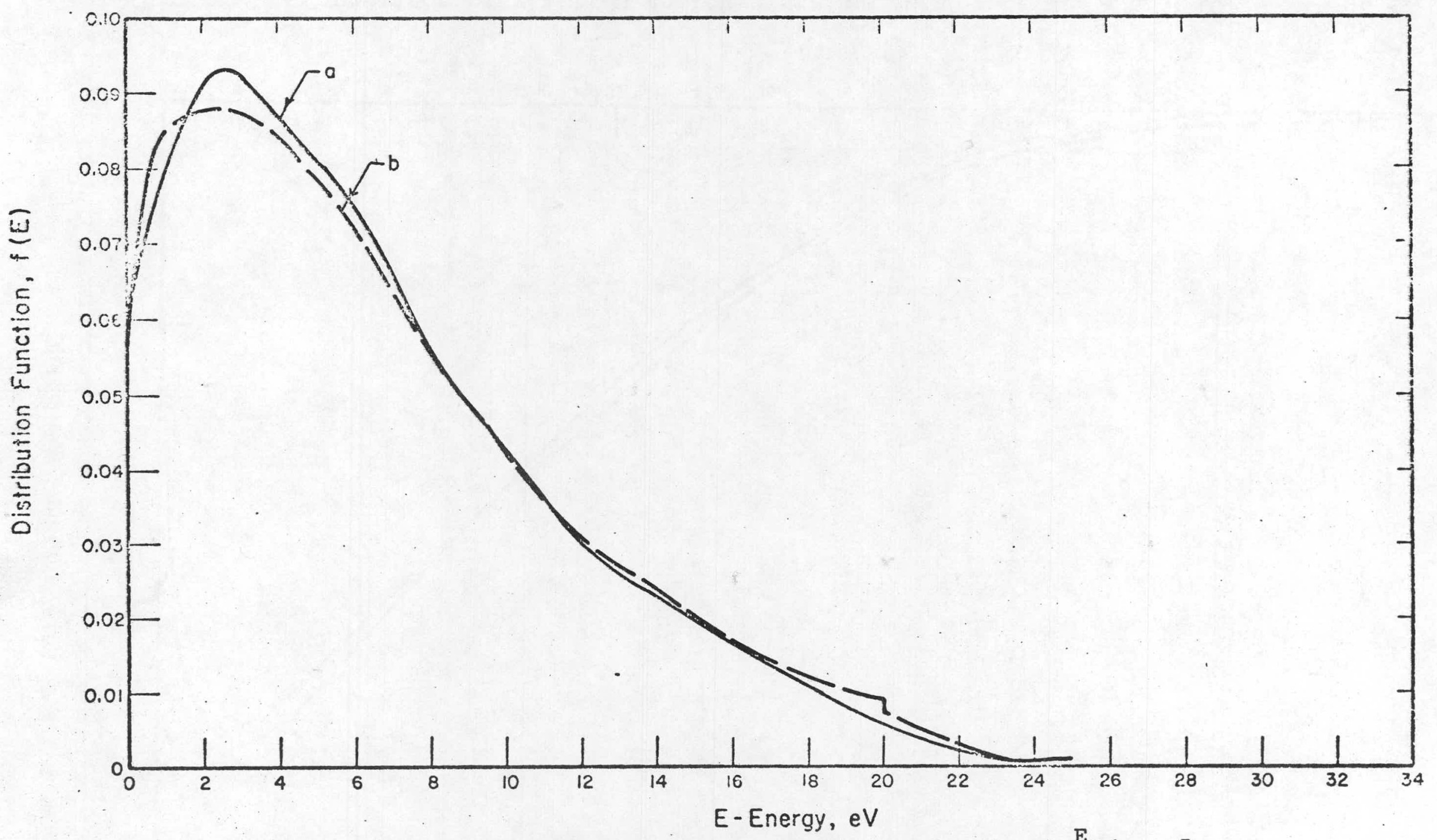

Figure 7. Comparison of electron energy distribution for He at $E_{f / p}=5$
a) present calculation $(\bar{E}=6.64)$

b) Heylen and Lewis' result $(\overline{\bar{E}}=6.51)$ 


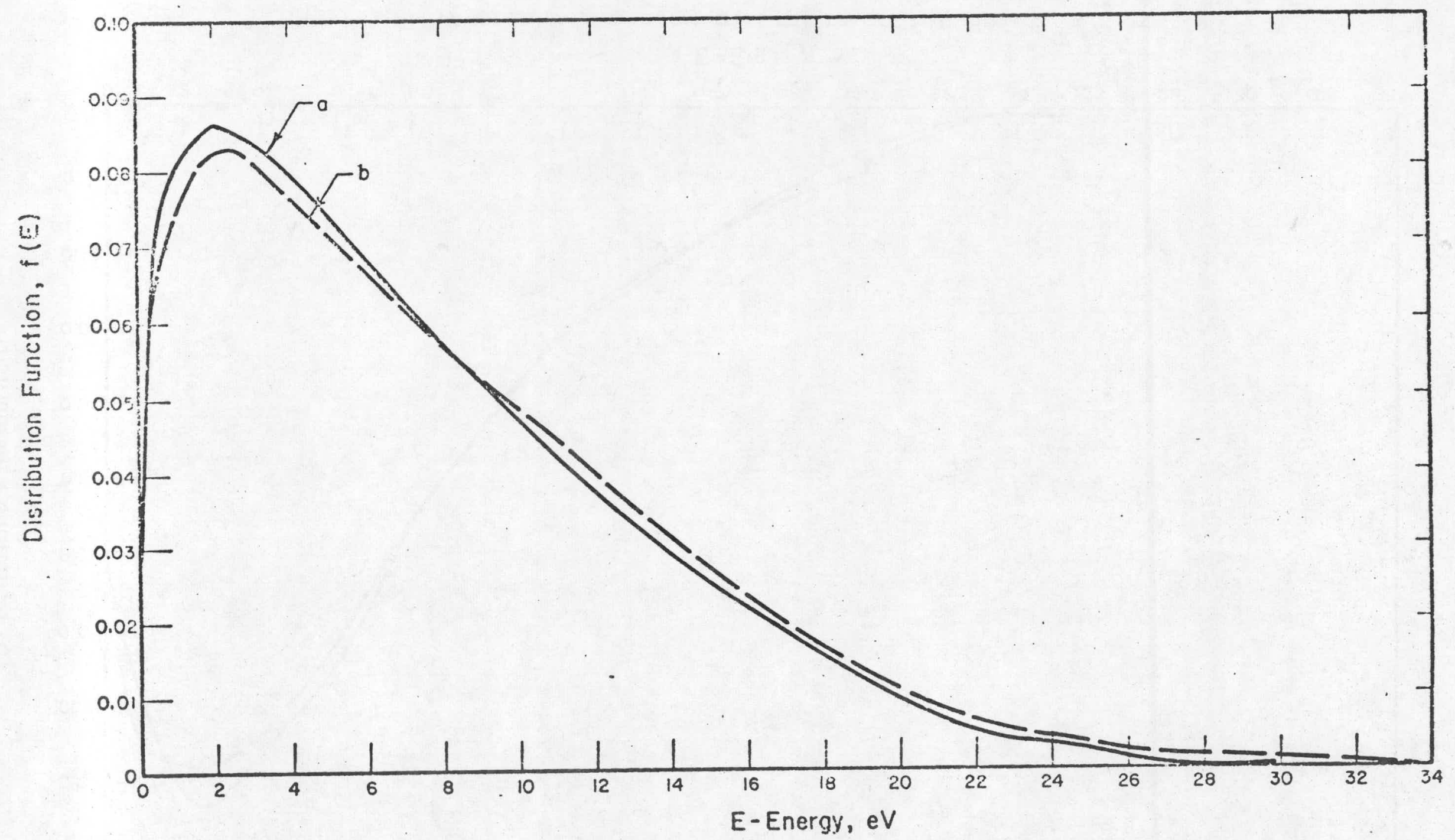
Figure 8. Comparison of electron energy distribution for He at $E_{f / p}=10$
a) Smit's result $(\bar{E}=7.7)$

b) present calculation $(\overline{\mathrm{E}}=7.83)$ 
the many approximations required in their analytic solutions. Also, some of the cross sections used in this calculations are experimental values, which may differ by some amount from that used for analytic solutions.

For the high energy region, (near the source energy) checks have been made against the analytic solutions reported by Lo and Miley (32). As shown in Figure 9, the general shape of the electron energy flux distributions are in reasonable agreement. In this case it was not practical to use the same cross sections as incorporated in the analytic solutions, and this may account for some of the differences observed. Also the analytic solutions are obtained for an infinite medium vs. a $1 \mathrm{~cm}$ slab in the present case. Thus the analytic solutions do not allow leakage, although this becomes important in the present calculation for low pressures. Lo and Miley's results are plotted in Figure 9 for the source energy $\mathrm{E}_{0}=1500 \mathrm{eV}$ and $E_{0}=500 \mathrm{eV}$, whereas the present results are for $E_{0}=$ $1000 \mathrm{eV}$. The plot shown is for the flux density (normalized) instead of energy number density. They are related as follows:

$$
\varphi(E)=f(E) \cdot V(E)
$$

where $\varphi(E)$ : electron flux density

$f(E)$ : electron number density

$V(E)$ : Electron kinetic velocity 


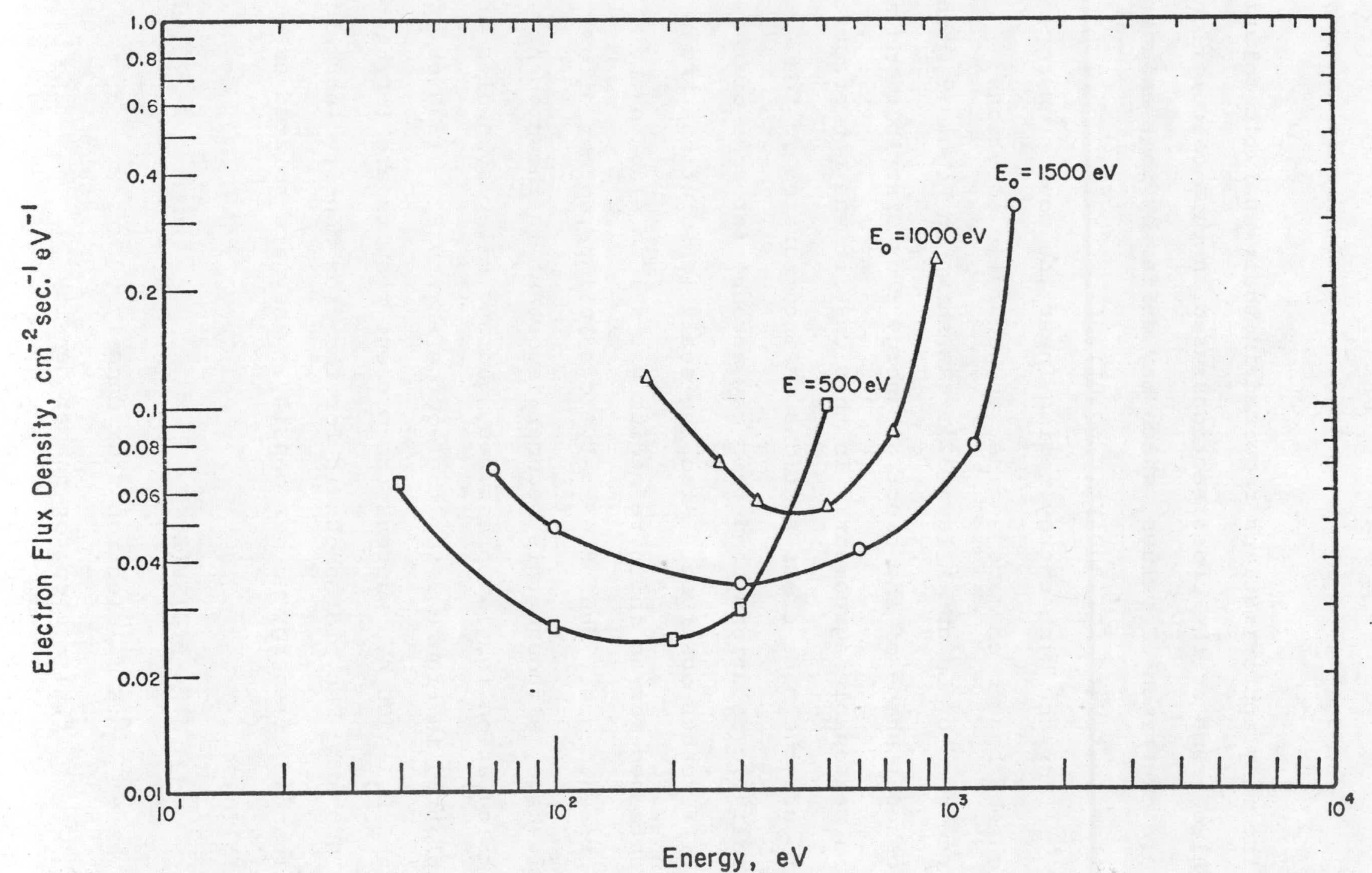

Figure 9. Comparison of the high-energy portion of flux density curve

a) $\mathrm{E}_{0}=1500 \mathrm{eV}$ and $\mathrm{E}_{0}=500 \mathrm{eV}$ : Lo and Miley's result

b). $E_{0}=1000 \mathrm{eV}$ : present calculations 
Since we only intend to compare general shapes of distribution, the normalizations used for the various curve, shown here are arbitrarily choosen for optimum display of information.

2. The Electron Energy Distribution Function without Electric Field

The calculations for electron energy distribution functions for helium with a high energy electron source, but no applied electric field (Figures 10 to 14 ) were carried out at two different pressures and various source rates. The slab thickness $D$ is chosen to be fixed at $D=1 \mathrm{~cm}$, which is equal to the actual diamter of cylindrical tube used in the laboratory (7). The two pressures are chosen to be $\mathrm{P}=10$ torr and $\mathrm{P}=760$ torr, corresponding to a typical low-pressure case and a normal atmospheric pressure.

We have chosen two different source energies $\left(E_{0}\right)$, one at $\mathrm{E}_{0}=1,000 \mathrm{eV}$ and the other at $\mathrm{E}_{0}=70 \mathrm{eV}$. These values roughly correspond to the highest energy and the average energy in the primary electron energy spectrum for MeV alpha-particle irradiation of helium as done in the experimental studies conducted at the University of Illinois (6).

The range of interest for the source rates for a boron coated tube irradiated in the Illinois TRIGA reactor lies between $10^{14}$ to $10^{18}\left(\# / \mathrm{cm}^{3}-\mathrm{sec}\right)$. However, results shown for source rates above about $10^{16}$ are questionable since the resulting ionization density becomes large enough that 
electron-electron collisions become important, and this process was neglected here.

The major quantity of interest here is the electron energy distribution function which were defined as $\mathrm{n}^{-}(E)$ in Chapter II. For simplicity, we represent the normalized distribution corresponding to $\mathrm{n}^{-}(E)$ by $f(E)$. This normalization is defined by:

$$
\int_{0}^{E_{0}} f(E) d E=1
$$

For the plasma system without electric field $\left(E_{f}=0\right)$, the distribution functions with source energy $\mathrm{E}_{\mathrm{o}}=1000 \mathrm{eV}$ are shown in Figures 10 and 11 for the pressure $p=10$ torr, and $p=760$ torr respectively. Similar distribution functions for $E_{0}=70 \mathrm{eV}$ are shown in Figures 12 and 13. An idealized Maxwellian distribution plus l/E tail are shown in Figures 10 to 13 for reference. (The Maxwellian plus $1 / E$ distribution was used as the initial distribution for these calculations. Note that since this curve is for reference only, it is arbitrarily normalized to a point on the Maxwellian curve and does not satisfy Equation 64.) For the range of source rates mentioned, the calculated energy distribution for helium (He) are reasonably Maxwellian at low energy region. The differences due to variations in the source rates show up at the high energy end, especially near the source energy, where the magnitudes seem to depend linearly on the values of source rate. This is somewhat 
deceptive, however, since the absolute magnitude at any point is due to the normalization. (This point is stressed again later in connection with Figure 24).

The fact that the distributions drop off rapidly for energies above about $20 \mathrm{eV}$ is attributed to the fact that the threshold energy for inelastic processes is $20 \mathrm{eV}$, above which energy the ionization and excitation processes bring more electrons into the low-energy region.

In order to investigate the change in distribution function at very high source rates, a calculation for $\mathrm{S}_{0}=$ $10^{22}$ particles $/ \mathrm{cm}^{3}-\mathrm{sec}$ was carried out. As mentioned earlier, the neglect of electron-electron collisions makes the accuracy of this calculation questionable. Still the trends are of interest, and it is seen from Figure 14 that the distribution curve changes drastically. In fact, it is no longer Maxwellian at the low-energy end. Due to the high source rate, more high-energy particles and more particles with energy in the range from $0.3 \mathrm{eV}$ to $20 \mathrm{eV}$ are present in the system.

As discussed earlier; the distribution curve drops sharply for energies above the excitation threshold energy (at $\mathrm{E}=20 \mathrm{eV}$ ) due to the dominance of ionization and excitation collision processes. At the higher pressure, the distribution curves decrease more rapidly compared to low pressure, since increased scattering slows the electrons down more rapidly. This effect is shown in Figures 15 and 16 , 


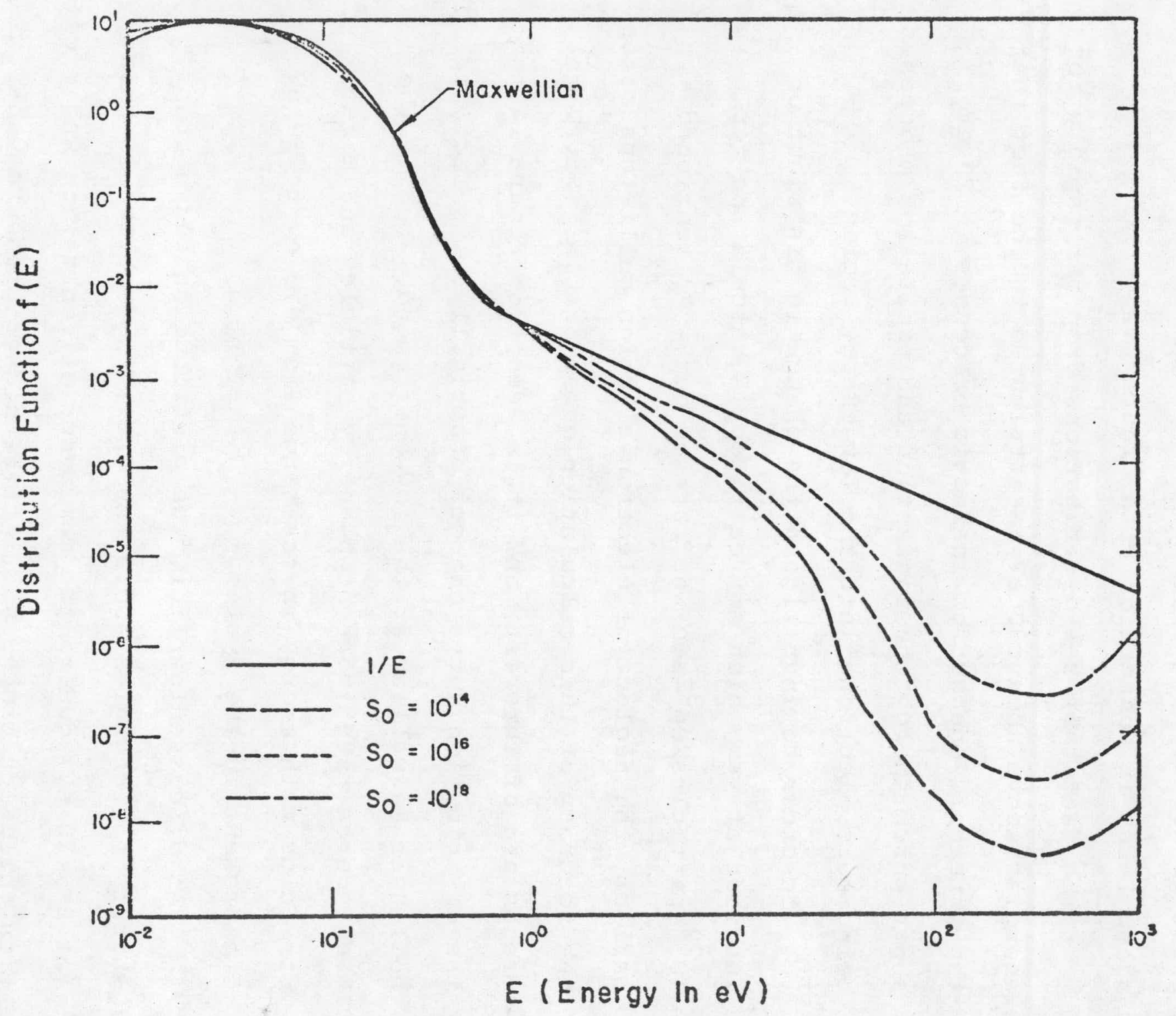

Figure 10. The electron energy distribution for He without an electric field but different source rates at $\mathrm{p}=10$ torr, $\mathrm{E}_{0}=1000 \mathrm{eV}$ and Maxwellian plus $1 / E$ distribution. 




Figure 11. The electron energy distribution for He without an electric field but different source rates at $p=760$ torr, $E_{0}=1000 \mathrm{eV}$ and Maxwellian plus $1 / E$ distribution. 


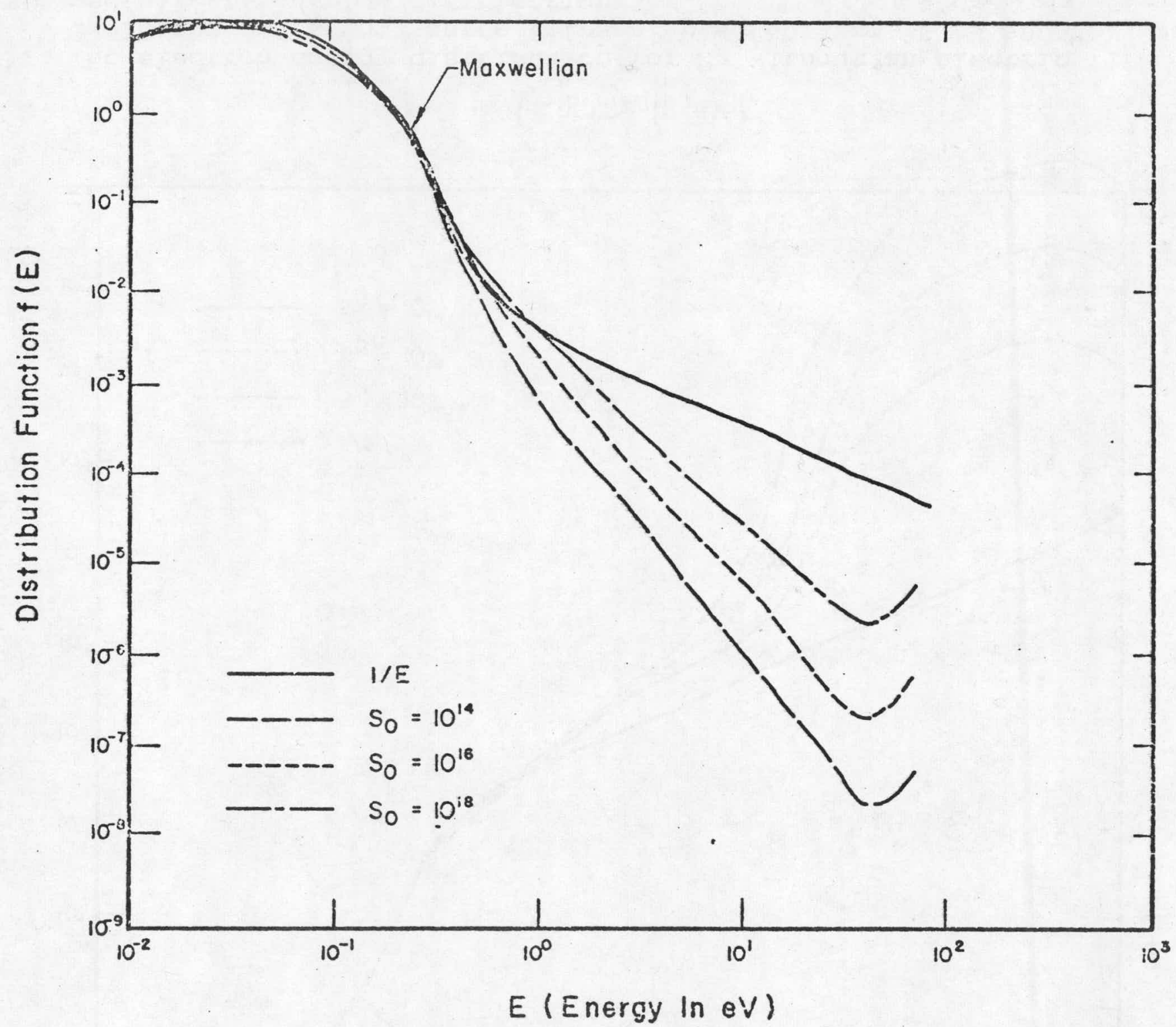

Figure 12. The electron energy distribution for He without an electric field but different source rates at $\mathrm{p}=10$ torr, $\mathrm{E}_{0}=70 \mathrm{eV}$ and Maxwellian plus $\mathrm{I} / \mathrm{E}$ distribution. 


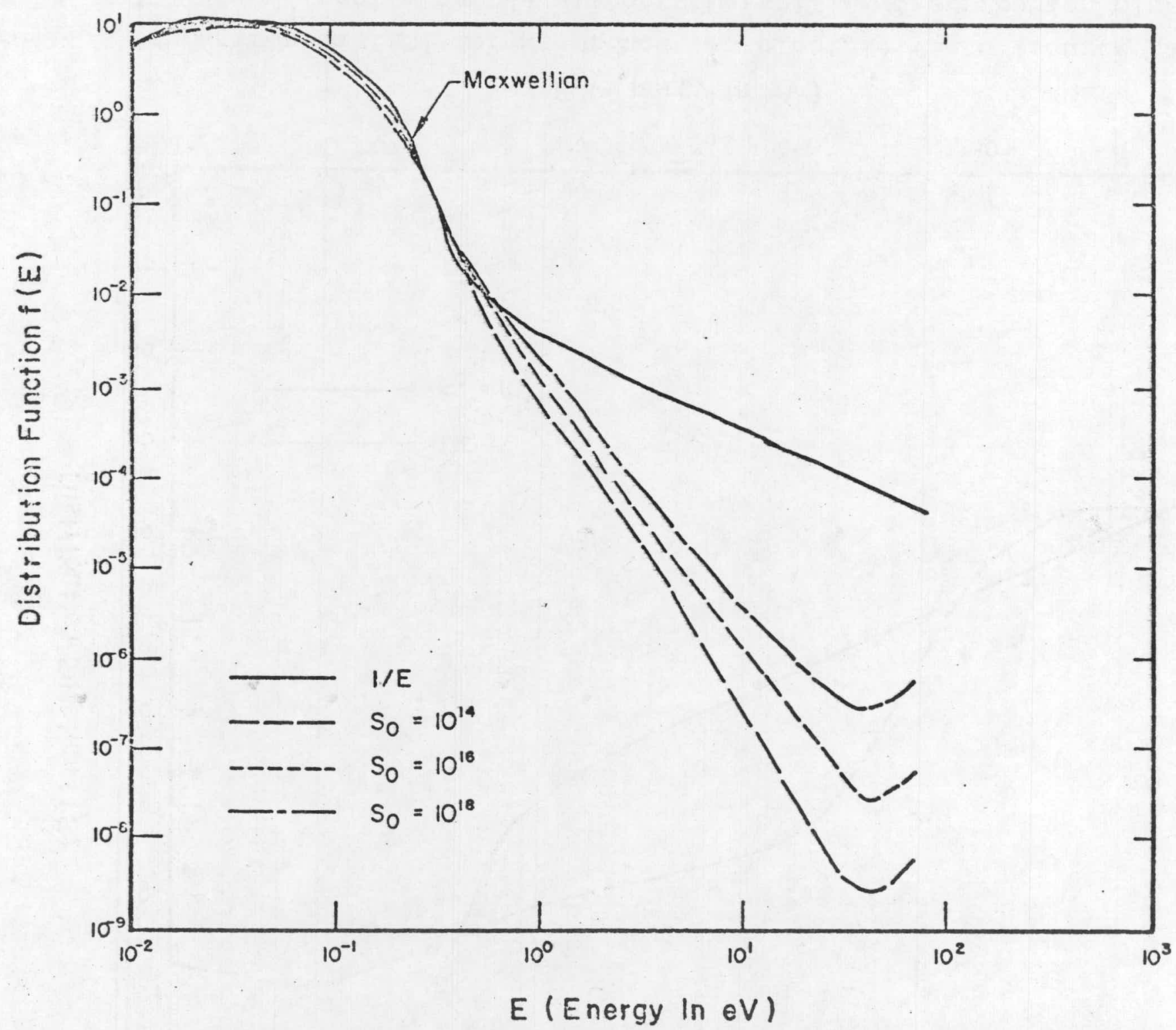

Figure 13. The electron energy distribution for He without an electric field but different source rates at $\mathrm{p}=760$ torr, $\mathrm{E}_{0}=70 \mathrm{eV}$ and Maxwellian plus $1 / E$ distribution. 


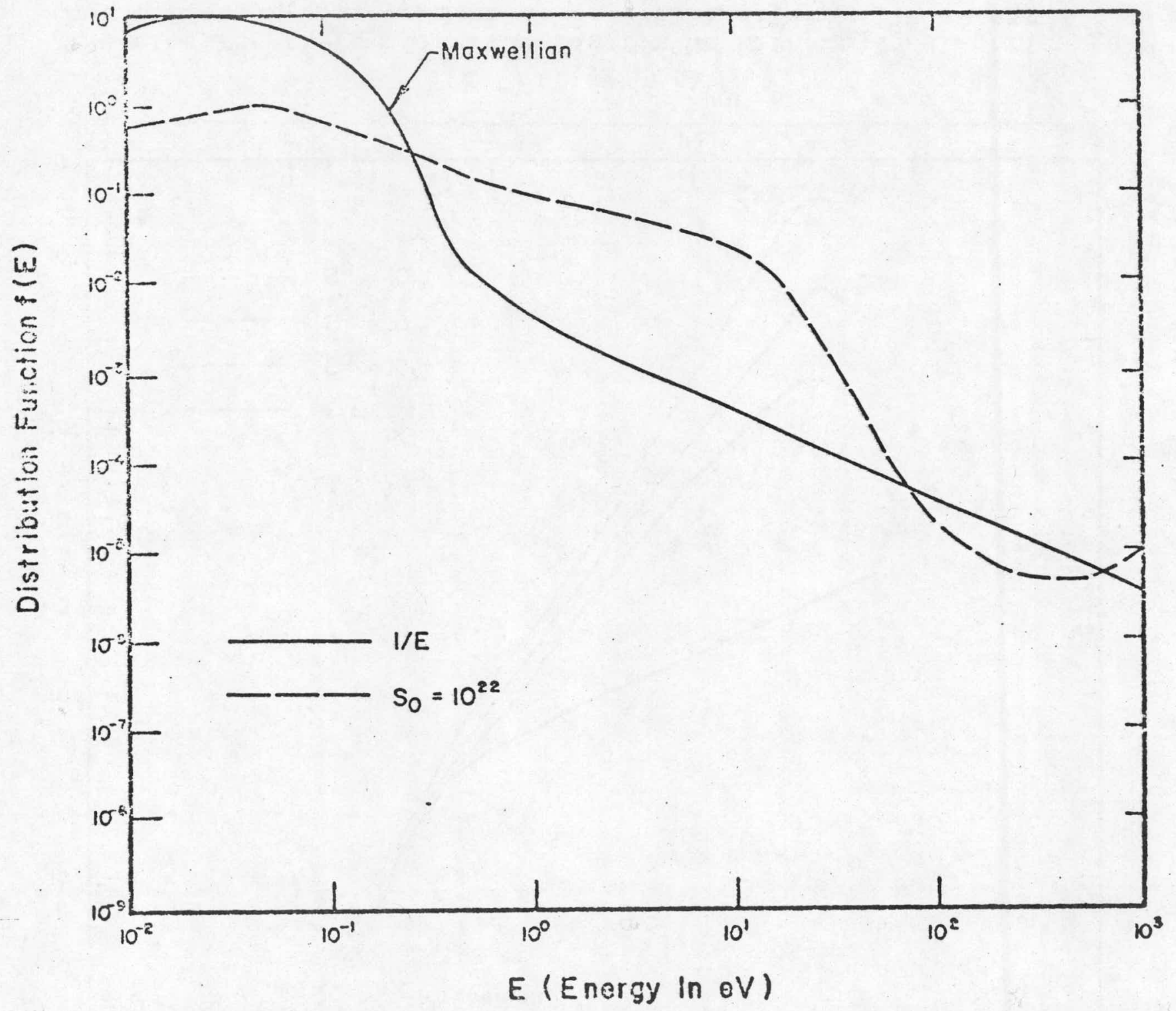

Figure 14. Comparison of $f(E)$ for He without an electric field (source rate $S_{0}=$ $\left.10^{22} \# / \mathrm{cm}^{3}-\mathrm{sec}\right)$ with the standard Maxwellian distribution plus $1 / \mathrm{E}$ tail. 


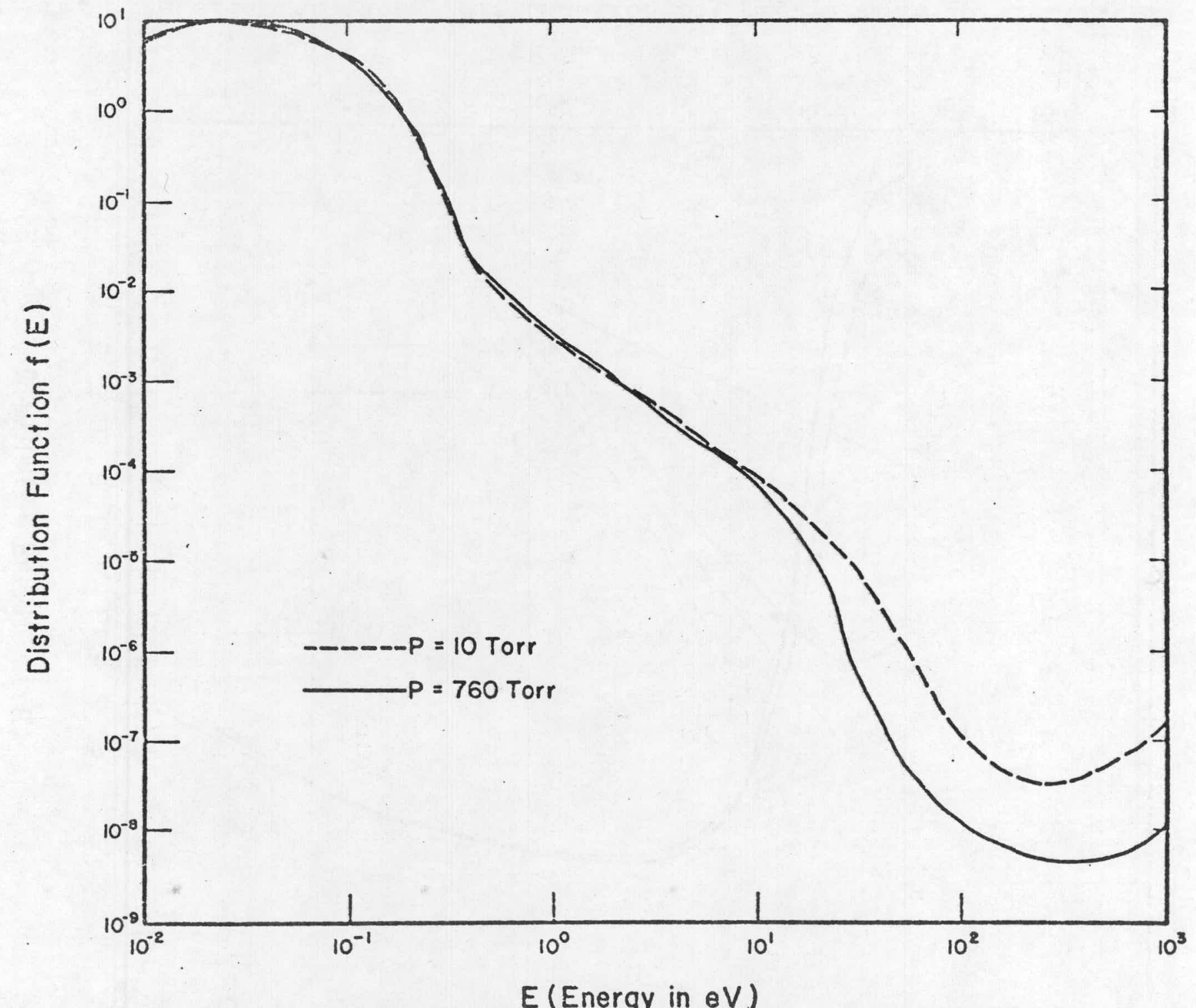

Figure 15. The electron energy distribution for He, without an electric field, for different pressure at $S_{0}=10^{16}, E_{0}=1000 \mathrm{eV}$. 


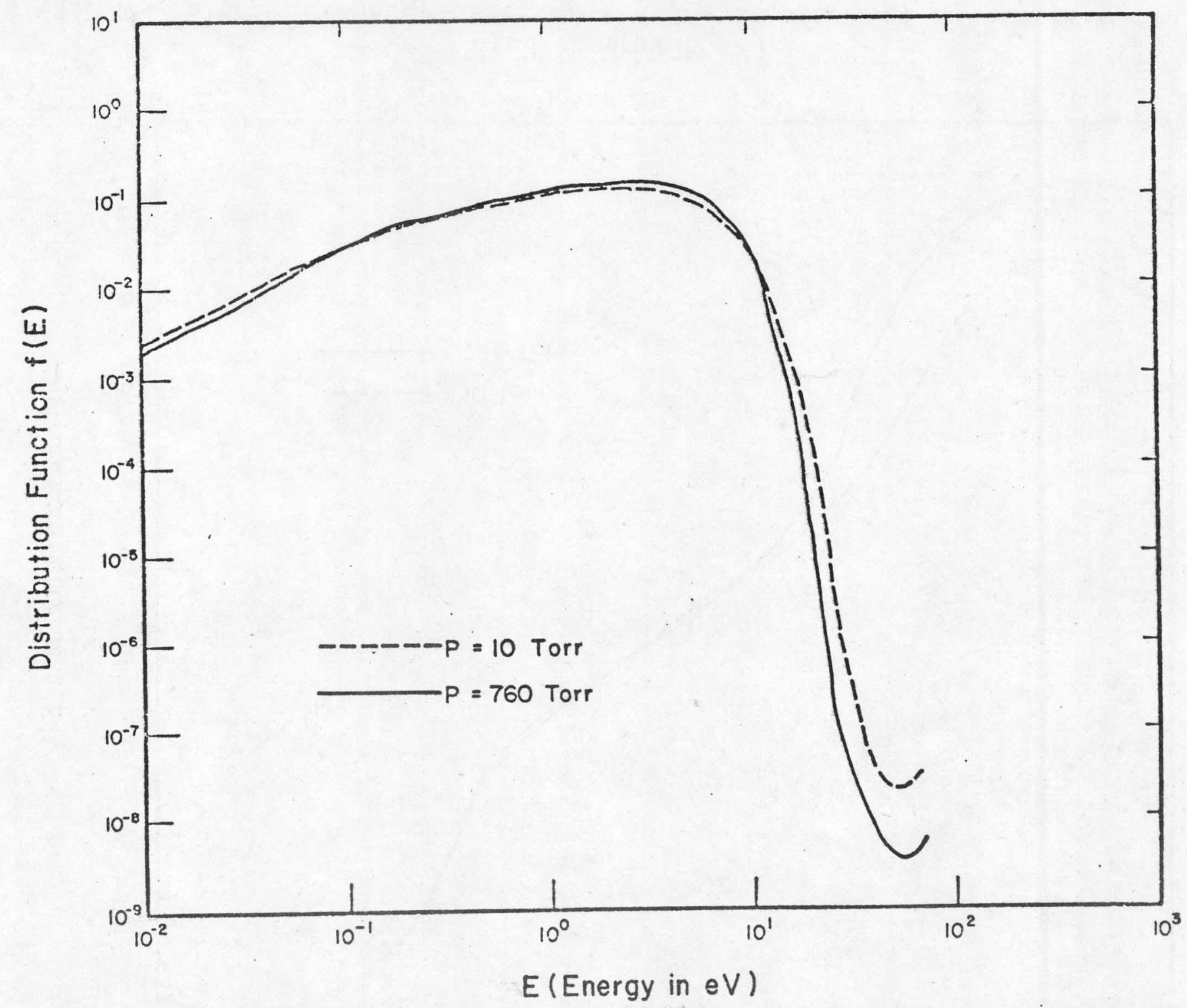

Figure 16. The electron energy distribution for He, without an electric field, for different pressure at $S_{0}=1016, E_{0}=70 \mathrm{eV}$. 
for the same source rate $s_{0}=10^{16}\left(\# / \mathrm{cm}^{3}-\mathrm{sec}\right)$ and source energy, but two different pressures. The reduction in magnitude of the high energy tail as the pressure increases is clearly illustrated.

In these calculations, the rate of both leakage and absorption (recombination) are strongly dependent on the pressure. At higher pressures (e.g., $p=760$ torr), very few particles leak out of the system, and the loss are dominated by the absorption process. For lower pressure ( $p=10$ torr), fewer particles are absorbed, while more escape or leak out of the system. Also, more particles escape in the high-energy region, but the absorped particles are mainly in the low-energy region.

The estimated major parameters are summarized in Table 1, which includes the average energy of the system ( $\bar{E})$, the number of positive ions $\left(\mathrm{N}^{+}\right)$and the W-values for various combinations of source rate $S_{O}$, source energy $E_{O}$ and pressure p.

The $W$-value is defined as the amount of energy required (input) to produce an ionization pair (positive ion and electron), or

$$
\text { W-value }=\frac{\left(N_{S} \cdot E_{0}-E_{T e S}-E_{T a b}\right)}{N_{z}}
$$

Where $\mathrm{N}_{\mathrm{S}}$ : the number of source particles introduced into the system during the period $\wedge t$.

$E_{\text {Tes }}$ : the total energy lost with escaping particles during the time $\Delta t$. 
Table 1. Calculated Parameter Values for the Plasma Without a Electric Field

\begin{tabular}{|c|c|c|c|c|c|c|}
\hline $\begin{array}{l}* \mathrm{P} \\
\text { (torr) } \\
\end{array}$ & $\begin{array}{c}E_{0} \\
\left(e^{\mathrm{V}}\right) \\
\end{array}$ & $\left(\# / \mathrm{Cm}^{\mathrm{S}}-\mathrm{sec}\right)$ & $\begin{array}{c}\bar{E} \\
(\mathrm{eV}) \\
\end{array}$ & $\begin{array}{r}\mathrm{N}^{+} \\
(\#) \\
\end{array}$ & W-value & Range \\
\hline 10 & 1000 & $10^{18}$ & 0.171 & $0.125 \times 10^{14}$ & $41 \cdots$ & -63 \\
\hline 10 & 1000 & $10^{16}$ & 0.157 & $0.125 \times 10^{13}$ & 35 & $\sim 53$ \\
\hline 10 & 1000 & $10^{14}$ & 0.111 & $0.125 \times 10^{12}$ & 49 & $\sim 58$ \\
\hline 760 & 1000 & $10^{18}$ & 0.167 & $0.583 \times 10^{15}$ & $32-$ & - 38 \\
\hline 760 & 1000 & $10^{16}$ & 0.123 & $0.446 \times 10^{14}$ & $40-$ & $\sim 65$ \\
\hline 760 & 1000 & $10^{14}$ & 0.104 & $0.67 \times 10^{13}$ & $35-$ & -54 \\
\hline 10 & 70 & $10^{18}$ & 0.104 & $0.125 \times 10^{14}$ & 50 & $\sim 62$ \\
\hline 10 & 70 & $10^{16}$ & 0.082 & $0.125 \times 10^{13}$ & 47 & -63 \\
\hline 10 & 70 & $10^{14}$ & 0.075 & $0.124 \times 10^{12}$ & $40-$ & -57 \\
\hline 760 & 70 & $10^{18}$ & 0.082 & $0.16 \times 10^{15}$ & $47-$ & $\sim 65$ \\
\hline 760 & 70 & $10^{16}$ & 0.075 & $0.141 \times 10^{14}$ & 42 & $\sim 58$ \\
\hline 760 & 70 & $10^{14}$ & 0.075 & $0.157 \times 10^{13}$ & $39-$ & -55 \\
\hline \multicolumn{7}{|c|}{ Maxwellian distribution } \\
\hline & & 0 & 0.05 & --- & & \\
\hline
\end{tabular}

*P: pressure, $E_{0}:$ source energy, $S_{0}:$ source rate, $\overline{\mathrm{E}}$ : average energy of the particles, $\mathrm{N}^{+}$: number of positive ions. 
$E_{\text {Tab }}$ : the total energy lost with recombining particles during the time $\Delta t$

$\mathrm{N}_{\mathrm{z}}$ : the total number of ionizations produced during the time period $\Delta t$.

As seen from Table 1 , the average energy $\bar{E}$ tends to be higher for higher source rates and higher source energy, and it is lower for higher pressures. However, the differences are in general very small. (All the average energies are consistently greater than that of a Maxwellian distribution). The values for ion density $\mathrm{N}^{+}$are roughly proportional to the square root of source rate, and are higher for higher pressures, virtually independent of source energy.

The W-values are quite sensitive to statistical fluctuations, and the ranges of variation obtained over the last 10 time cycles in a given calculation are shown in Table 1. The W-values obtained here do not show any clear dependence on the system parameters, thus variation indicated may be totally due to the statistical fluctuations. This is not too surprising since the $W$-value is known to be roughly independent of particle energy, etc. The W-value for helium obtained experimentally by Jesse and Sadouski (34) is 43. A reasonable value to choose for the W-value from Table 1 would be 50 , which is little bit higher than the experimental result. The reason for this discrepancy is not clear, but it may be due to small inaccuracies in the ionization-excitation cross section employed here. The W-value is, by definition, quite sensitive to the details 
of the ratio of the ionization to the excitation cross section as a function of energy.

3. The Electron Energy Distribution Function with an Applied Electric Field

The calculated electron energy distribution functions in He with an applied electric field $\left(E_{f} / p=10\right)$ are plotted in Figures 17 to 21 , in a manner similar to that in the previous section. All these calculations are carried out at the electric field to pressure ratio $E_{f} / p=10$. Smit's result (without source or $s_{0}=0$ ) are plotted together with present calculations at given pressure and source energy $\left(p=10,760\right.$ and $\left.E_{0}=1000 \mathrm{eV}, 70 \mathrm{eV}\right)$, with three different source rates. It is observed that the distribution functions are highly non-Maxwellian. For the source rate in the range $10^{14} \# / \mathrm{cm}^{3}-\sec$ to $10^{18} \# / \mathrm{cm}^{3}-\mathrm{sec}$, the distribution curves at low energy end are quite close to the smit's results (1I). At the high energy end, they have a shape similar to the curves of no electric fields. Again differences due to the source rate show up at high-energy region. Below $10 \mathrm{eV}$, all the distribution functions fall on the top of each other, and they begin to spread out as the energy increases above $10 \mathrm{eV}$. For high pressures or low source energy, more difference (between $s_{0}=0$ and $s_{0} \neq 0$ ) show up at high-energy end than that of low pressure and/or high source energy. Again at very high source rates $s_{0}=10^{22}$ $\left.\ddot{f} / \mathrm{cm}^{3}-\mathrm{sec}\right)$ the distribution function changes appreicably. 
In Figure 21, the comparison is made with a very high source rate $\left(\mathrm{S}_{\mathrm{O}}=10^{22} / \mathrm{cm}^{3}-\mathrm{sec}\right) \therefore$ a nd with one in the region of interest ( $S_{0}=10^{16}, p=760$ torr). For very high source rate, the distribution function changes quite a lot; it still preserves the general shape, but at low-energy end, it is much smaller in amplitude. (However, as stressed earlier, the high source rate calculation is questionable due to the neglect of electron-electron interactions.)

The changes in these distribution functions due to different pressuresare also shown in Figures 22 and 23. The observed trends are similar to those in the previous section for the electric field $\mathrm{E}_{f}=0$ case.

So far, the normalized distribution functions $f(E)$ have been displayed. However, to stress the fact that the absolute magnitudes of the curves depends strongly on the source (shapes have received our interest to this point), Figure 24 shows the unnormalized electron energy distribution functions for He (with electric field or $E_{f} \neq 0$ ) are plotted, with $E_{f} / p=10, p=10$ torr, $E_{0}=1000 \mathrm{eV}$, and three different source rates $\left(\mathrm{s}_{\mathrm{o}}=10^{14}, \mathrm{~s}_{\mathrm{o}}=10^{16}, \mathrm{~s}_{\mathrm{o}}=10^{18}\right)$. The magnitude (unnormalized) distribution is defined as

$$
N^{-}(E)=N^{+} \cdot f(E)
$$

where $\mathrm{N}^{+}$: number of positive ions

$f(E)$ : normalized distribution

The values of $\mathrm{N}^{+}$are $0.24 \times 10^{15}, 0.24 \times 10^{14}$, and $0.24 \times 10^{13}$ corresponding to the three source rates $s_{0}=10^{18}, s_{0}=10^{16}$ 


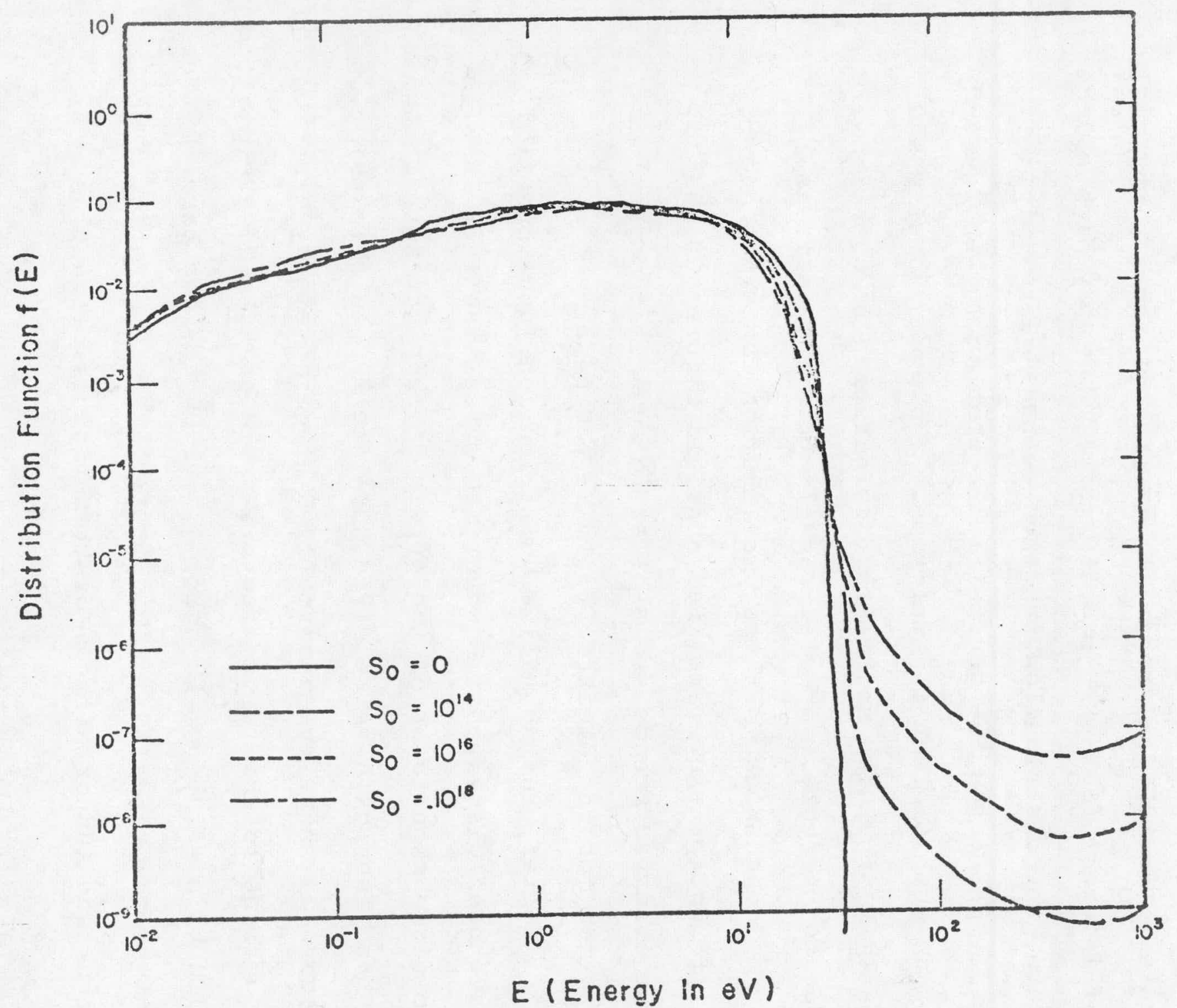

Figure 17. The electron energy distribution for He with an electric field at $E_{f / p}=10, p=10$ torr, $E_{0}=1000 \mathrm{eV}$. 


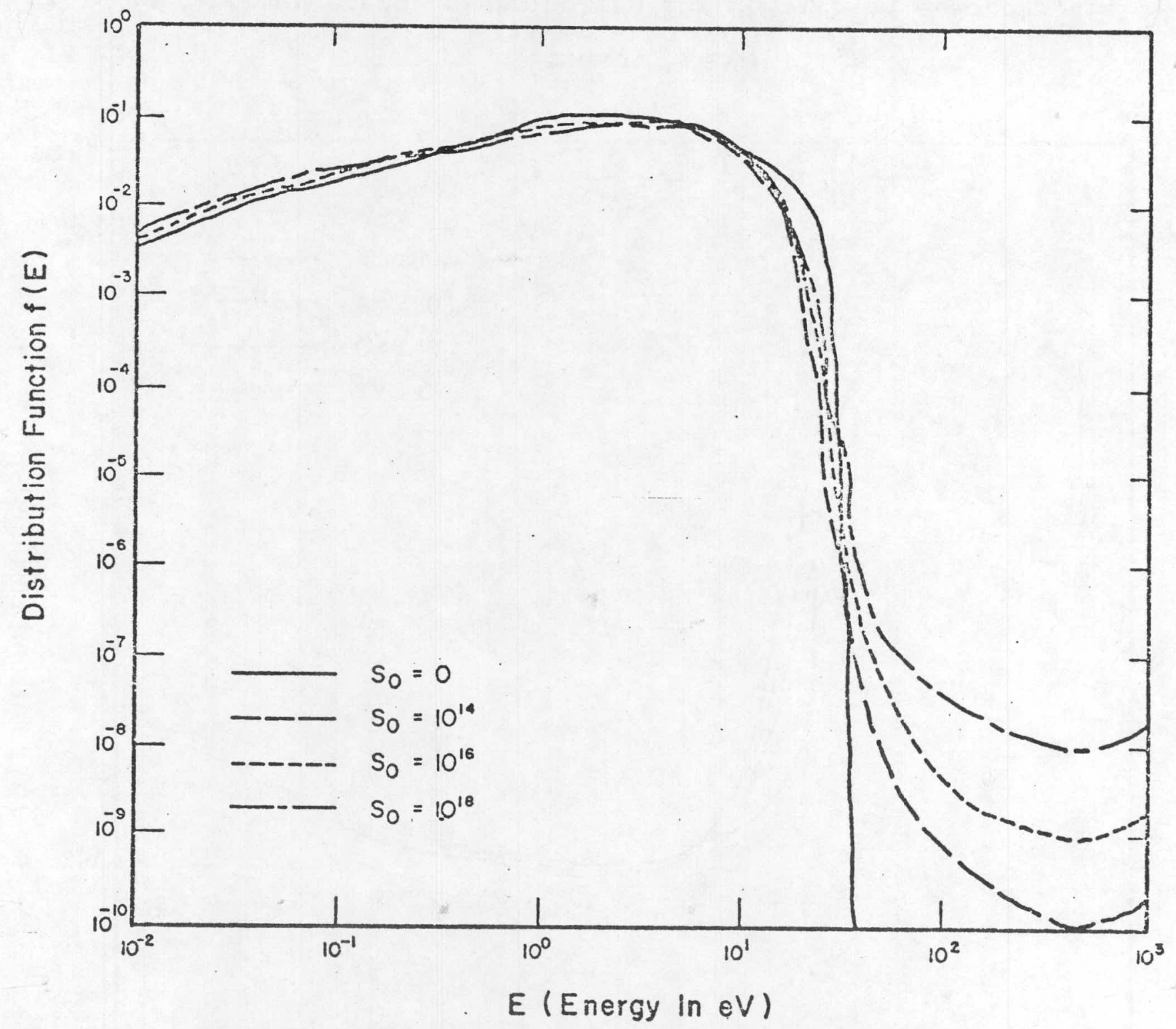

Figure 18. The electron energy distribution for He with an electric field at $E_{f / p}=10, p=760$ torr, $E_{0}=1000 \mathrm{ev}$. 


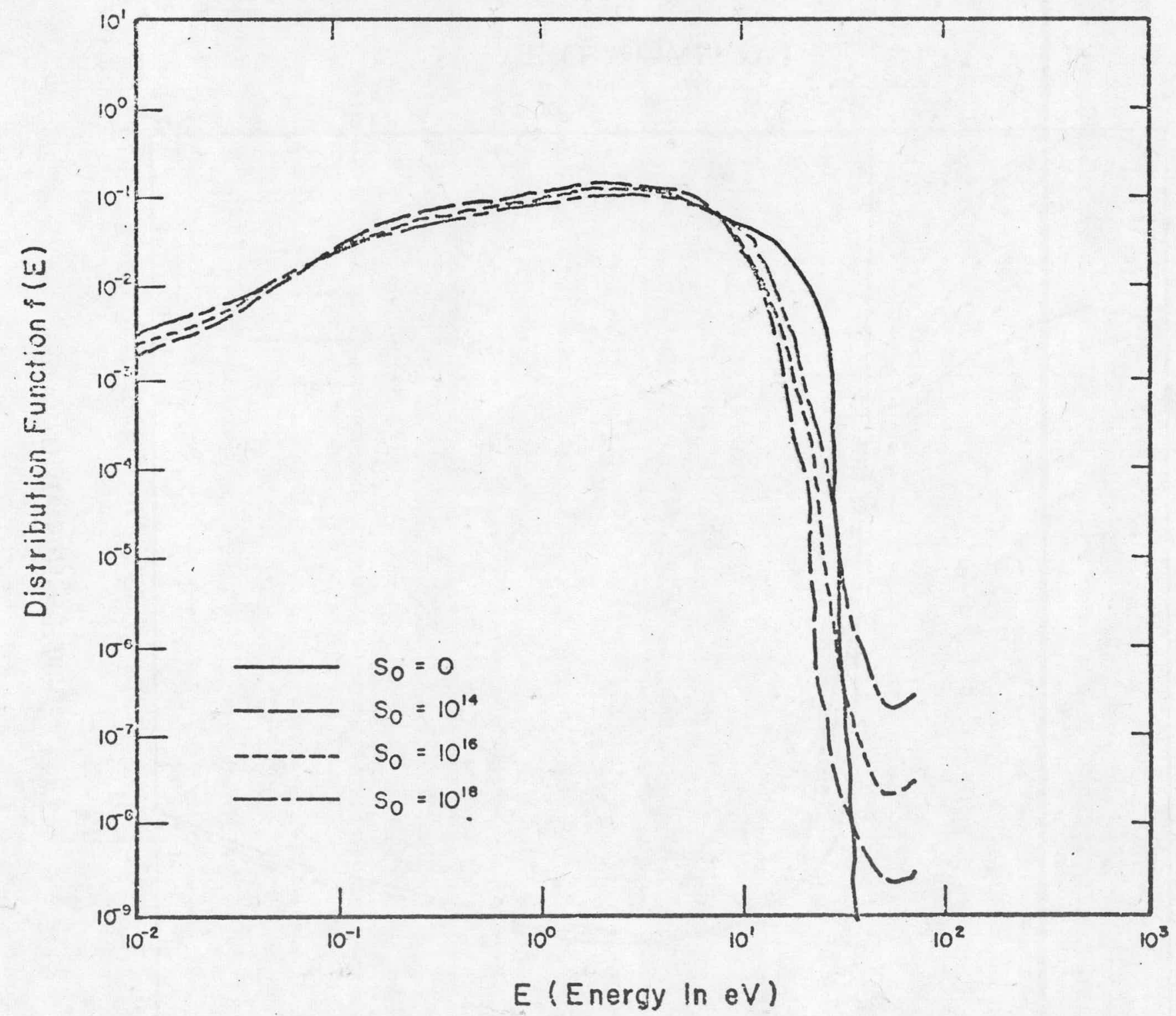

Figure 19. The electron energy distribution for He with an electric field at $E_{f / p}=10, p=10, E_{0}=70 \mathrm{eV}$. 


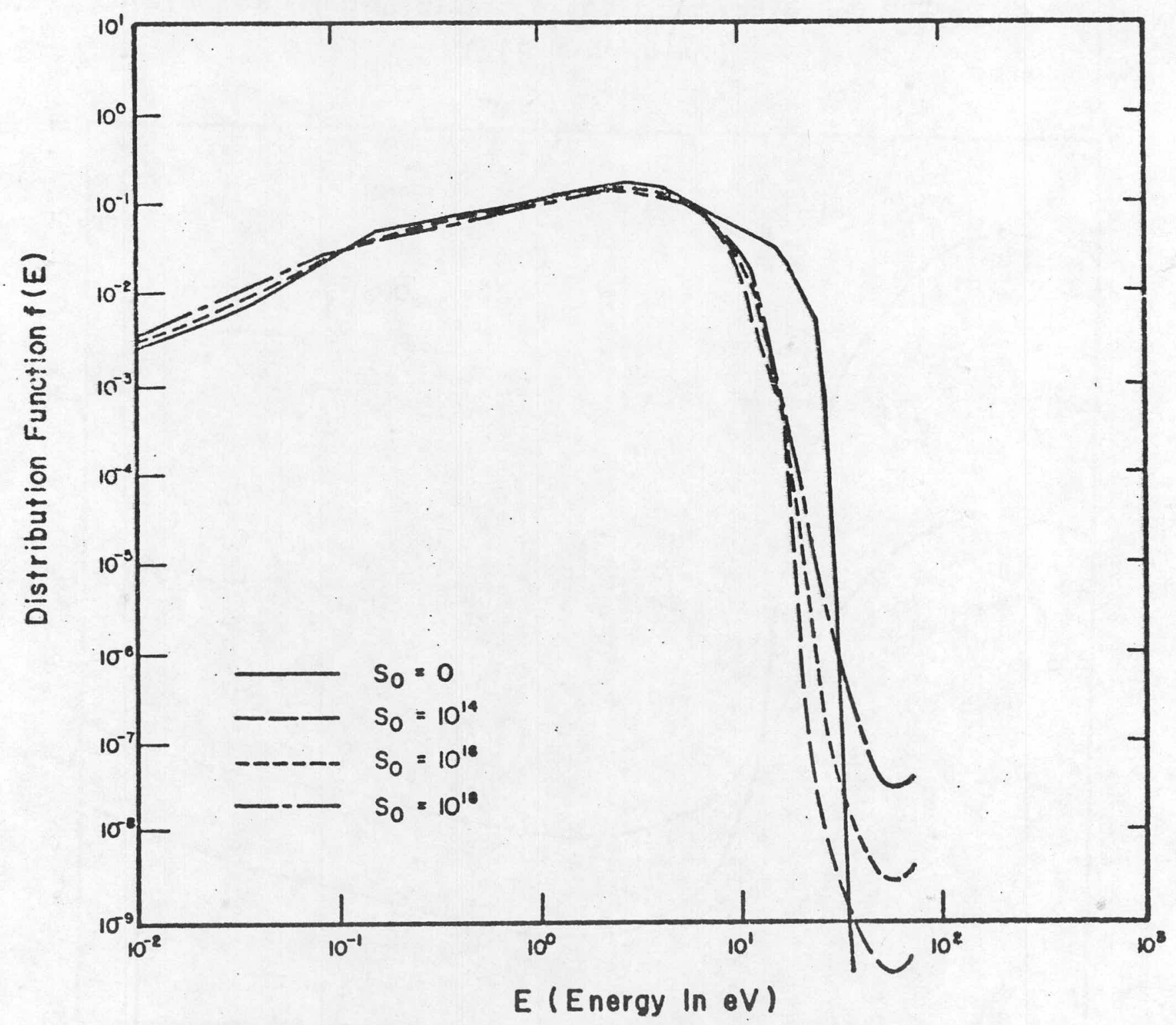

Figure 20. The electron energy distribution for He with an electric field at $E_{f / p}=10, p=760$ torr, $E_{0}=70 \mathrm{eV}$. 


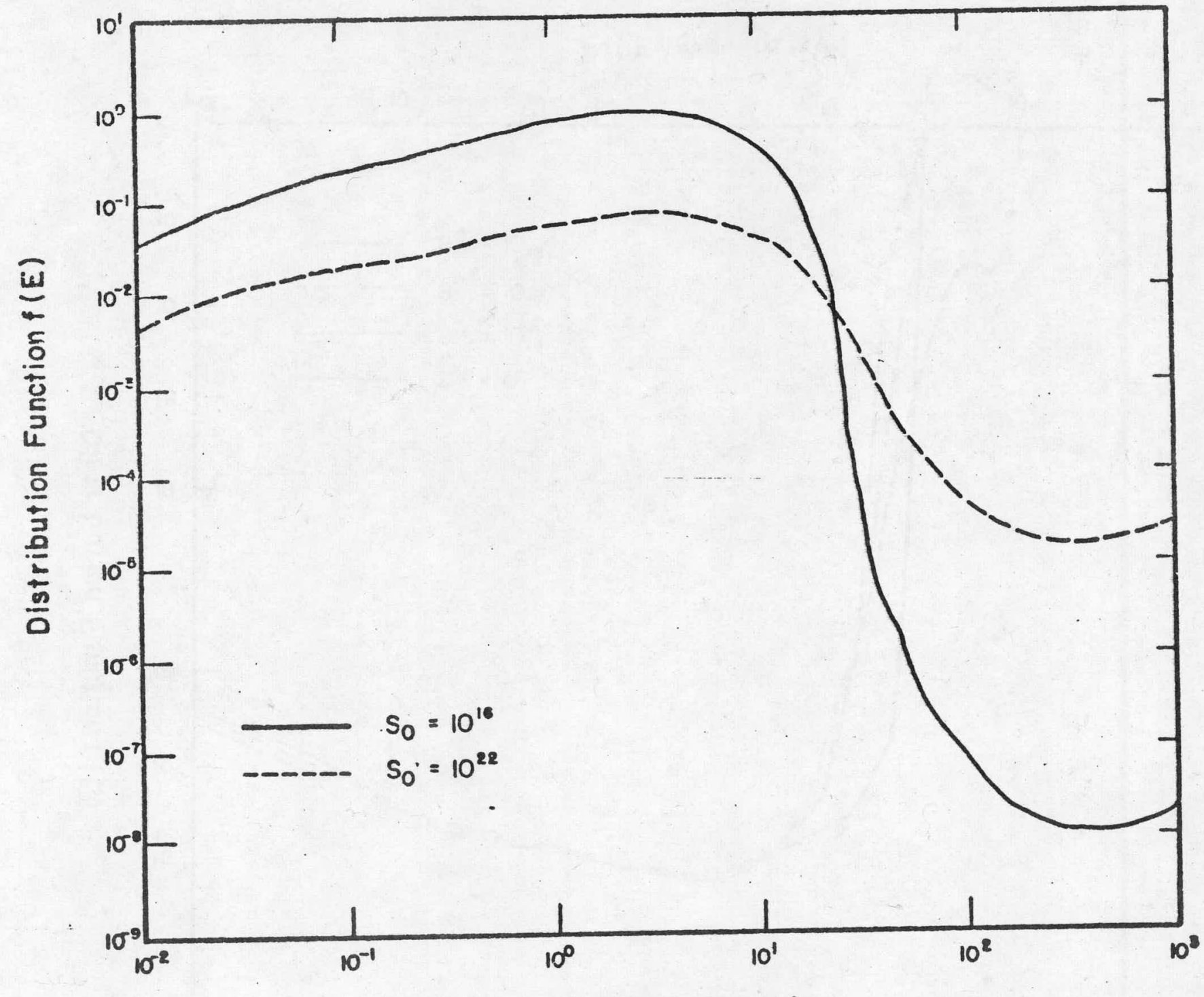

$E$ (Energy in eV)

Figure 21. The electron energy distribution 'function at $E_{f / p}=10, p=760$ torr for $s_{0}=10^{22}$ and $s_{0}=10^{16}\left(\# / \mathrm{cm}^{3}-\mathrm{sec}\right)$. 


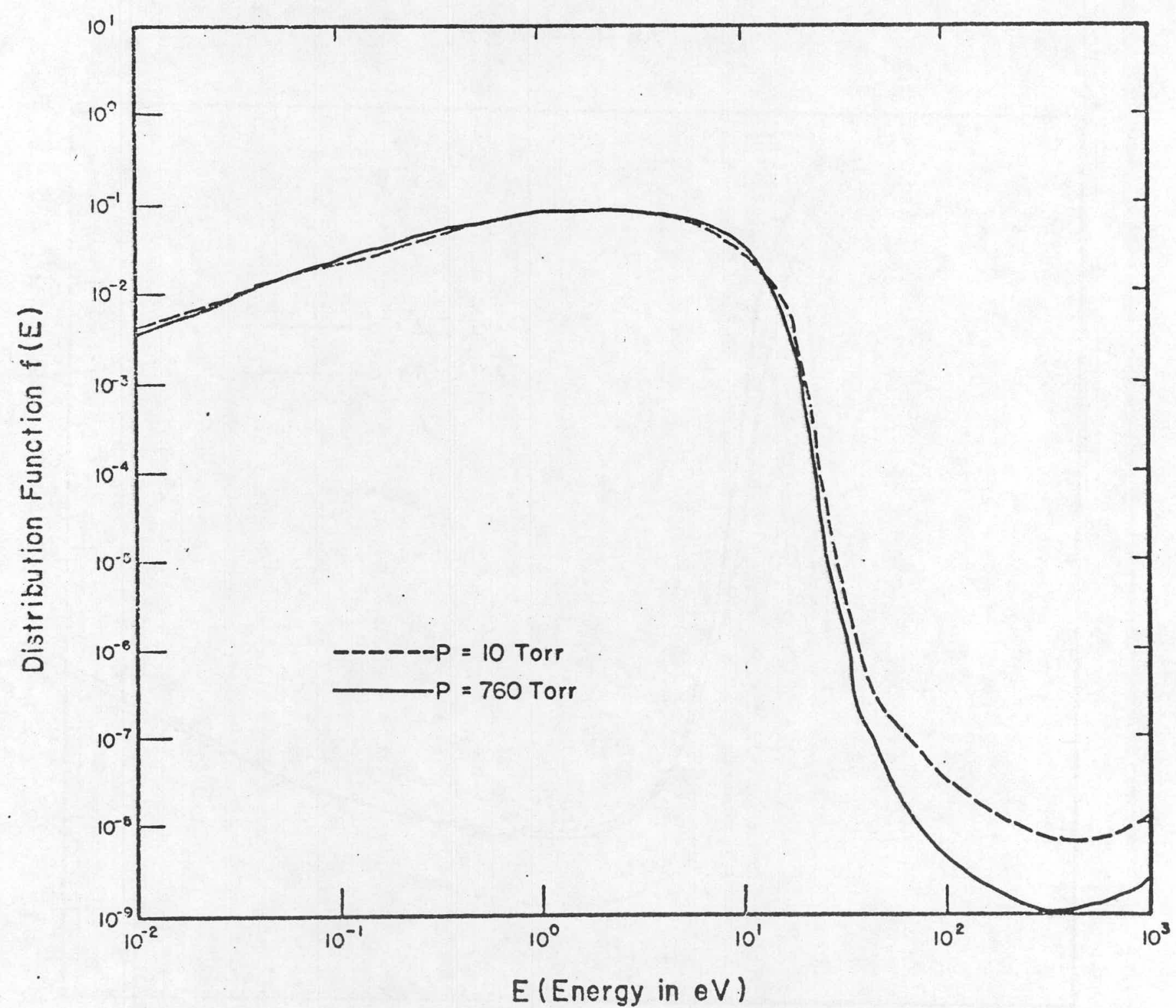

Figure 22. The electron energy distribution for He, with an electric field at $S_{0}=10^{16}, E_{0}=1000 \mathrm{eV}$. 


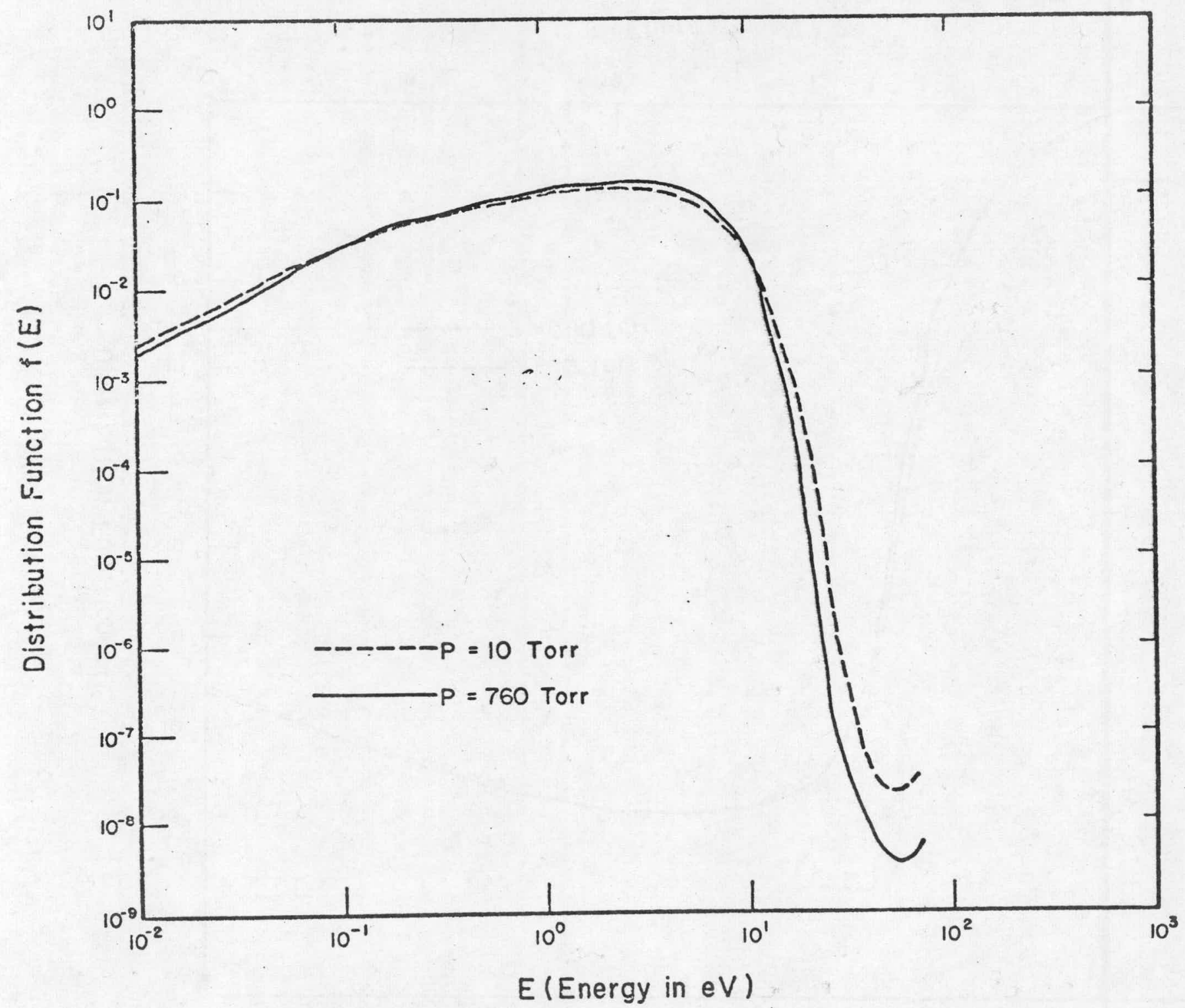

Figure 23. The electron energy distributions for He, with an electric field at $s_{0}=10^{16}, E_{0}=70 \mathrm{eV}$. 


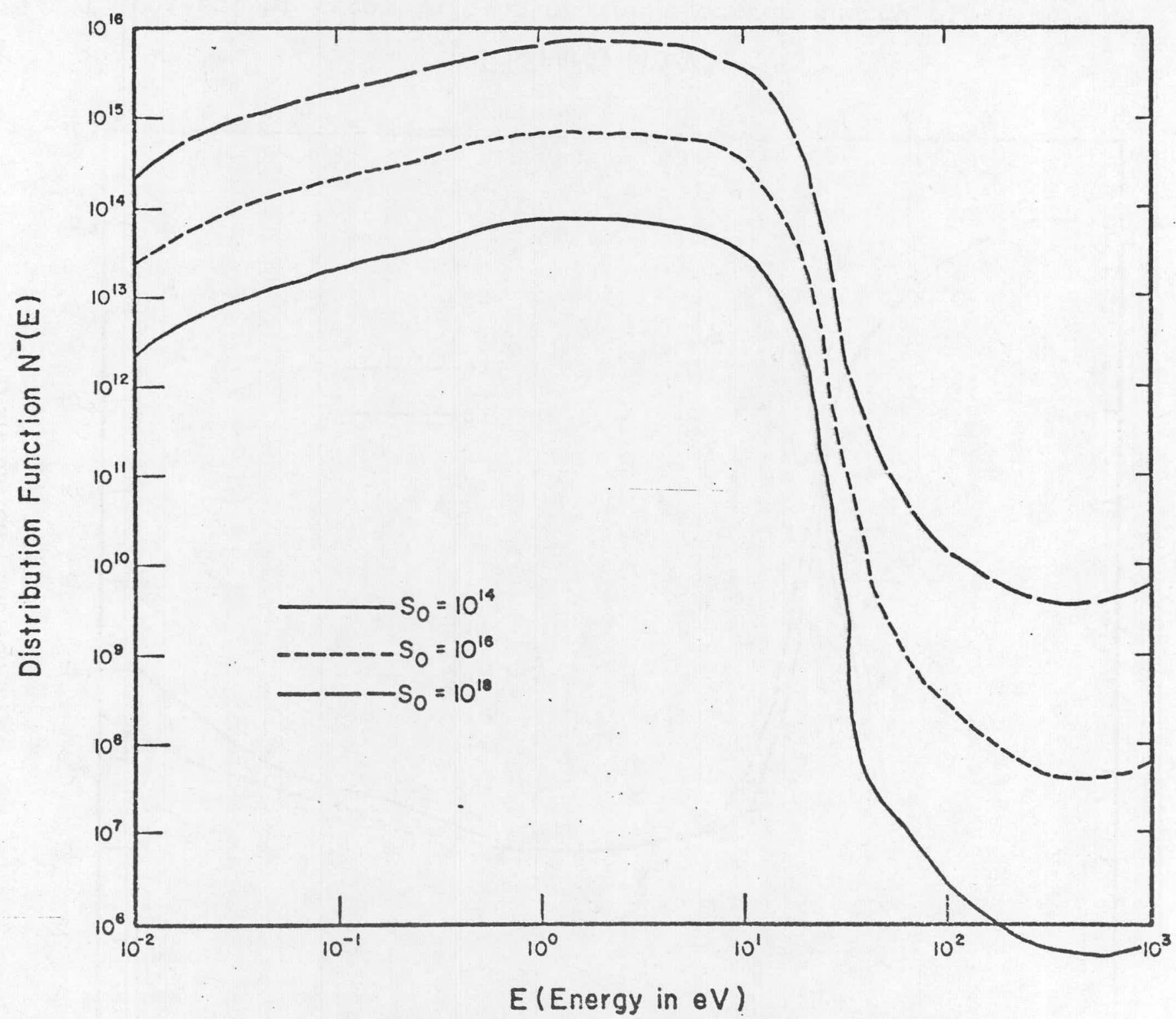

Figure 24. The unnormalized electron energy distribution for He at $E_{f / p}=10, p=10$ torr, $E_{0}=1000 \mathrm{eV}$. 




Figure 25. The effect of recombination on the electron energy distribution a) with normal recombination cross section $\times 100$ 
and $S_{0}=10^{14}$, respectively.

In order to show the effect of recombination, a normal

case is compared in Figure 25 to a calculation where the absorption cross section was arbitraxily increased 100 times. It is observed that the major effect comes in at low-energy region. More and more electrons recombine as they thermalize and hence, the lower energy region of the distribution is depleted. Since recombination does not occur at high energies, this region is not affected.

The calculated major parameter values for the system with an electric field are summarized in Table 2. As expected on physical grounds the values of the average energies for this case are mich higher than the corresponding values with no electric field (compare to Table 1). However, other general trends are the same, e.g. the value of the average energies are slightly higher for higher source rate and/or higher source energy, but are lower for higher pressures. For comparison the average energy $(7.7 \mathrm{eV})$ found by Smit for an electric field and $E_{f / p}=10$ but with no source present is included in Table 2. It is in general higher than the present values. The reason is not entirely clear. At first thought the additional high-energy source electrons might be expected to raise the average energy. However, as found by T. Ganley (37), the presence of highenergy electrons to produce ionization allows the electrons in the Maxwellian region to decrease in energy, and as a result the overall average may actually decrease. 
Table 2. Calculated Parameter Values for Plasma with Applied Electric Field $\left(E_{f / p}=10\right)$

\begin{tabular}{|c|c|c|c|c|c|}
\hline $\begin{array}{l}* \mathrm{P} \\
\text { (torr) }\end{array}$ & $\begin{array}{c}E_{\circ} \\
(\mathrm{eV}) \\
\end{array}$ & $\begin{array}{c}\mathrm{S}_{3} \\
\left(\# / \mathrm{cm}^{3}-\mathrm{sec}\right) \\
\end{array}$ & $\begin{array}{c}\bar{E} \\
(\mathrm{eV}) \\
\end{array}$ & $\begin{array}{c}\mathrm{N}^{+} \\
(\#) \\
\end{array}$ & $\begin{array}{l}\text { Modified } \\
\text { W-value range }\end{array}$ \\
\hline 10 & 1000 & $10^{18}$ & 8.1 & $0.24 \times 10^{15}$ & $35 \sim 44$ \\
\hline 10 & 1000 & $10^{16}$ & 7.5 & $0.21 \times 10^{14}$ & $34 \sim 54$ \\
\hline 10 & 1000 & $10^{14}$ & 7.3 & $0.24 \times 10^{13}$ & $37.5 \sim 60$ \\
\hline 760 & 1000 & $10^{18}$ & 7.0 & $0.23 \times 10^{17}$ & $37 \sim 50$ \\
\hline 760 & 1000 & $10^{16}$ & 6.6 & $0.204 \times 10^{16}$ & $30 \sim 51$ \\
\hline 760 & 1000 & $10^{14}$ & 5.4 & $0.24 \times 10^{15}$ & $34 \sim 57$ \\
\hline 10 & 70 & $10^{18}$ & 5.86 & $0.21 \times 10^{15}$ & $31 \sim 35$ \\
\hline 10 & 70 & $10^{16}$ & 5.18 & $0.21 \times 10^{14}$ & $34 \sim 50$ \\
\hline 10 & 70 & $10^{14}$ & 4.46 & $0.213 \times 10^{13}$ & $31 \sim 56$ \\
\hline 760 & 70 & $10^{18}$ & 5.7 & $0.284 \times 10^{16}$ & $55-46$ \\
\hline 760 & 70 & $10^{16}$ & 5.0 & $0.26 \times 10^{15}$ & $39 \sim 48$ \\
\hline 760 & 70 & $10^{14}$ & 4.3 & $0.24 \times 10^{14}$ & $32 \sim 49$ \\
\hline \multicolumn{6}{|c|}{ Smit's distribution } \\
\hline & & 0 & 7.7 & --- & -- \\
\hline
\end{tabular}

*p: pressure; $E_{0}=$ source energy: $S$ : source rate;

$\mathrm{E}$ : average energy; $\mathrm{N}^{+}$: number of positive ions. 
The values of $\mathrm{N}^{-4}$ are roughly proportional to the square root of the source rate (recall Equation 33) and are independent of the pressure and source energy.

Strictly speaking, the definition of the W-value for the field case should include an allowance for energy gained to electrons in the electric field which then result in ionization. There is no way to include this energy transfer in the present case, so the values listed in Table 2 are based on the ion pair production divided by the energy input with the high-energy source electron alone (no field contribution). This is, then the same formulation (Eq. 65) as that of no field case, and we lable the result as the "effective" or "modified" W-value. Again these values fluctuate considerably, so the ranges observed in the last 10 time cycles are given. As would be expected from the neglect of the field contribution, the Wvalues in this table are in general smaller than that of no field case. As in the earlier calculation, no general trend can be observed, as so far as the dependence of the parameters ( $\left.E_{O}, S_{O}, p\right)$ are concerned, and this is consistent with the earlier observations and the literature which indicate that the $W$-values are fairly insensitive to these parameters. We have concluded so far that, the distribution functions for non-field case $\left(E_{f}=0\right)$ are more or less assume a Maxwellian distribution in the low-energy region for source rates below $10^{18}$. However, when an electric field 
is added, the distribution shifts to that found by Smit in the low-energy region. This is, in effect, the well-known Druyvesteyn distribution. It is significant that the addition of a source adds a tail to this distribution, but it does not drastically change the low-energy region. By intuition, it might be expected that smit's distribution (with electric field or $\mathrm{E}_{f} \neq 0$ ) would change back to a Maxwellian distribution as the electric field approaches zero. In order to display the transition region, the distribution functions for smaller electric field $\left(E_{f / p}=1\right)$ are plotted in Figure 26 together with the calculated distribution functions at $E_{f / p}=10$ and $E_{f}=0$. The highenergy tail of the distribution curve are not significantly affected by reducing the electric field $E_{f}$, but the distribution function in the low energy region starts to approach a Maxwellian distribution as expected.

\section{Conclusion on Monte Carlo Results}

Based on the Monte Carlo solutions we obtained so far, ,we can make the following comments.

1. For the normal range of source rate $\left(s \leq 10^{18}\right)$, where the neglect of electron-electron interactions remains valid, the Maxwellian plus $1 / E$ distribution is a good initial approximate distribution for the no electric field case. Likewise the Smit (or Druyvesteyn) plus $1 / E$ distribution is a good approximate iritial distribution to be used for the system with an electric field present. 


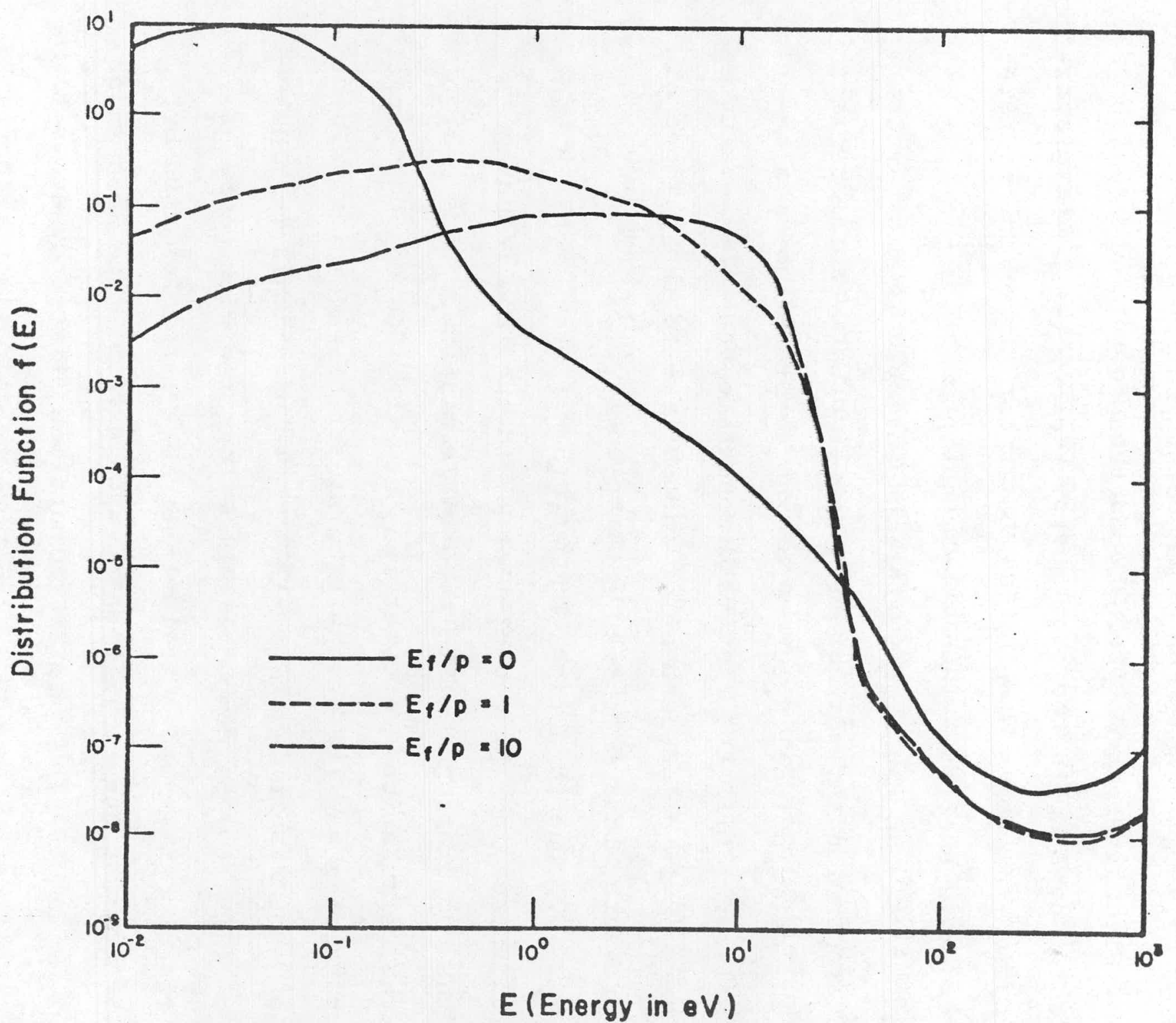

Figure 26. The electron energy distribution for He at $p=10$ torr, $s_{0}=10^{16}$
for $E_{f / p}=0, E_{f / p}=1$ and $E_{f / p}=10$. 
2. The dependence of the distribution functions (with or without electric field) upon the pressure and source rate only shows up in the high-energy region, where the distribution functions have higher tails for higher source rate, and have smaller values for higher pressures.

3. The range of variations observed in the W-value: calculation reflects statistical fluctuations which limit the calculational accuracy of the absolute numerical Wvalues. Still the results demonstrate that the $\mathrm{W}$-value is more or less independent or insensitive to the changes of pressure, source rate and source energy.

4. Due to a tight budget, the primary emphasis was on increasing the computation efficiency and reducing the computation time to a possible minimum. Thus most of the calculations were carried out with 2000 to 5000 actual particles and for any particular set of parameter values, and the results were generally obtained within 10 minutes of (IBM 360/75) computer time. Compared with hours of computer time and 5 to 10 times more particles used by others for the similar calculations (e.g., see References 3,4 , and 9) the gain in computation efficiency seems to be significant. Of course, a complete comparison is not possible since all of these calculations achieved different accuracies in the final results.

5. The present results also show close agreement with the analytic solutions of Lo and Miley $(32,33)$ on the 
107

following points:

a. The shape of high-energy portion of the distributron curves are in reasonable agreement as shown in Figure 9.

b. The high-energy part of the distribution function is not changed by the presence of an electric field for field values $E_{f / p} \leq 10$.

c. With an electric field, the low-energy part of the distribution function agrees with Sit's result; and this part is not affected appreciably by source rates $<10^{18}$ particles $/ \mathrm{cm}^{3}-\mathrm{sec}$. 
CHAPTER VI

CONCLUSIONS

\section{A. Prelude}

The application of Monte Carlo techniques to solve particle transport problems is of fundamental interest in many fields of physics and engineering; especially when analytic and/or numerical solutions of the basic governing equations are too complex to be practical. Yet direct application of Monte Carlo techniques to simulate individual interactions is sometimes prohibitively costly because of the large amount of computational time required. Thus techniques for improving the efficiency are required. Various existing variance reduction techniques can be used, but they are problem dependent. Thus techniques that are efficient for one type of problem might not be as effective for other types of problems. Thus such techniques must be judiciously selected and modified for the particular problem under consideration. One basic underlying principle which applies to any Monte Carlo calculation is: Apply as much known information as one can (given in analytic and/or numerical form) to reduce the uncertainties of the problem. Whenever possible Monte Carlo experiments should be checked and replaced by exact theoretical analysis to reduce uncertainties.

The Monte Carlo experimentalist has to exercise ingenuity in distorting and modifying problems in the pursuit 
of variance-reduction techniques. Although Monte Carlo methods are general devices, still a great deal of work depends upon the individual's originality to create special methods or numerical schemes to suit their needs. Unlike the physical experimentalist, the mathematical experimentalist using Monte Carlo Methods has the advantage that his experimental material consists of mathematical objects which can be distorted, controlled, and modified more easily. The greatest successes of the Monte Carlo method have arisen where the basic mathematical problem itself consists of the investigation of some random processes. However, there are exceptions involving deterministic problem-solution of boundry value problems and partial differential equations. The solutions of these problems are closely connected with the characteristics of certain random diffusion type processes (or can be converted to such type of processes), therefore these problems are reduced to the modeling of such processes. Thus the model and the special techniques developed in present research are quite general in scope, and it should be possible to apply them to many classes of Monte Carlo calculations with a certain amount of modification.

B. Summary of Present Work

In this research study, we have devised a general mathematical model for the particle transport or diffusion 
type plasma problems which have inherent nonlinear properties due to recombination. Many special variance reduction techniques are incorporated into the system. The model can be used for calculating both time-dependent and steady-state nonlinear transport problems provided that suitable methods exist to calculate, predict, and correct the important parameters which dominate the nonlinearities. A wide range of nonlinear problems can be formulated and handled in this way. The principle idea, the piecewise linearized predictcorrect model, is a general, simple, and efficient approach for solving nonlinear problems. In principle, most nonlinear problems can be cast in terms of the basic ideas and algorithms of present work. This represents an extension of earlier works of Musha and Itoh (8), Thomas (9), and Fleck Jr. (11). As for the amount of computation time and efficiency involved, the present study has made significant improvements. Some of the improvements are a natural consequence of the large storage space available in modern computer systems. Additional improvements come from a few 'simple, but effective, special techniques incorporated into the present model. As described in Chapter II, these include:

1) The initial distribution - straightforward particle tracing procedures would require following every simulated particle from its birth (from source) with initial energy $E_{0}$ at $t=t_{0}$ to the end of its life time history. This type of simulation would require hundreds 
of cycle times to achieve a final steady state condition. Instead, we start with an apprixmately steady-state system by guessing an initial particle energy distribution. Then we simulate the system by tracing particles starting with energy corresponding to this input distribution. This is equivalent to skipping over the initial transient time cycles and greatly reduces the computation time and increases the calculation efficiency.

2) The application of the negatively-correlated variates (antithetic variates) technique greatly reduces statistical fluctuations with a minimum number of simulating particles (machine particles).

3) The introduction of a weighting scheme and an artificial game of chance (using weighted cross sections) contributed some reduction in calculation time and resolved some practical difficulties.

4) For the system with an applied electric frield, the exact formulation for a parabolic flight path was used to advance the particles in larger steps than would have been possible using the small-step linear approximation employed by Musha and Itoh (8) and also by Thomas (9). This improves the efficiency appreciably.

Throughout the entire calculation, one basic balance equation (Eq. 30) played a very important role. It merely states that at steady state, the source is balanced by losses. 
By means of this equation, we predict the nonlinear parameter values and provide checks for convergence of the solution. The need for large amounts of storage space for Monte Carlo calculations no longer creates serious problems or drawbacks on modern large computer systems, such as was the case as recent as seven or ten years ago. In addition, techniques exist for further reducing the storage requirement. In the present study, we suggest a technique to eliminate major storage requirements, and use only a small amount of storage as a temporary buffer storage (described. later).

In other types of numerical solutions, e.g., in finite difference solutions of the diffusion equation, the step size $\Delta t$ is restricted by stability requirements. However, in this present model, $\Delta t$ is only restricted by the degree and amount of nonlinearities present in the system. This restriction is far less stringent.

\section{Practical Limitations and the Cures}

It was necessary to solve several difficulties in the present simulation study in order to provide practical solutions. We shall describe them item by item.

1) The electron energy distribution is the quantity of major interest. The difference between the magnitudes of its maximum and minimum non-zcro values is of the - order $10^{3}$. This is due to high density in the low-energy region (thermal energy) and the relatively low density 
in the high-energy region (near the source energy). It is unrealistic to hope to produce reasonable numbers of particles in the low-density (high-energy) region and at the same time have $10^{9}$ times as many in the lowenergy region. This problem is resolved by combining the following techniques:

a) Russian Roulette and splitting.

b) Weight factors which produce fractional particles.

C) Logarithmic energy intervals. The number of particles in each energy class interval $\Delta E_{i}$ is directly proportional to $f\left(E_{i}\right) \cdot \wedge E_{i}\left(f\left(E_{i}\right):\right.$ number density at $\left.E_{i}\right)$. By using logarithmic energy intervals, we have small intervals at low energy region and large intervals at high energy so that the product $f\left(E_{i}\right) \cdot \Delta E_{i}$ assumes a practical magnitude.

2) The total number of simulating particles (machine particles) $\mathrm{N}_{\mathrm{T}}$ is limited by the storage requirement and the economy of the calculation (computing time is proportional to $\mathrm{N}_{\mathrm{T}}$ ). We have to set an upper limit (Nmax) on the total number of simulating particles in any time cycle. During each time cycle of duration $\Delta t, N_{s}$ source particles are introduced into the system, and $\mathrm{N}_{z}$ secondary particles are produced (by ionization). At the same time $\mathrm{N}_{\text {es }}$ particles escape from the system, and $\mathrm{N}_{\mathrm{Ab}}$ particles are lost due to recombination.

There are two conditions to be satisfied at all times: 

a) $\mathrm{N}_{\mathrm{C}}+\mathrm{N}_{\mathrm{Z}}+\mathrm{N}_{\mathrm{S}} \leq \mathrm{N}_{\mathrm{T}} \leq \mathrm{N}_{\max }$
b) $\mathrm{N}_{z}+\mathrm{N}_{\mathrm{S}} \simeq \mathrm{N}_{\mathrm{es}}+\mathrm{N}_{\mathrm{AB}}$

where $\mathrm{N}_{\mathrm{C}}$ : the number of census particles (background particles).

Condition a) insures that total number of particles in the system is always under the limit ( $\max$ ). Condition b) indicates that a particle balance should be satisfied at steady state.

In the program, there are two equivalent problems to be considered. One is the balance of the machine particles [corresponding to condition (a)], and the other is the balance of simulated particles [weighted particles, corresponding to condition $(b)]$. Both conditions must be satisfied simultaneously. Condition (a) allows a certain amount of leeway since the number of particles that survive the integration cycle (time cycle) and hence require storage will be less than $\mathrm{N}_{C}+\mathrm{N}_{z}+\mathrm{N}_{S}$. Here $\mathrm{N}_{\mathrm{S}}$ is chosen to be an input number, the weights of these particles are determined by the source rate. If condition (a) can not be met, $N_{S}$ is taken to be

$$
N_{S}=N_{\text {max }}-N_{C}-N_{z}-J Z-I
$$

where $\mathrm{JZ}$ is the number of spatial zones. It has been shown (12) that Equation 68 insures the stabilization of census population without overly reducing the number of source particles. 
The proper selection of the energy dividing line (EDI) for Russian Roulette and splitting can insure the satisfaction of condition (b). Also as mentioned in earlier chapters, the balance factor (BF) is used to speed up convergence and force condition (b) to be satisfied sooner.

3) In any Monte Carlo calculation, finite samples are used to estimate or simulate the behavior of a large parent population. This is the main source of statistical fluctuations (variance or uncertainty). In the present simulating system, less than 5000 particles were used to simulate approximately $10^{14}$ physical particles. This creates the problem of matching the values of input and estimated output quantities. Due to the huge scaling factor $\left(10^{14} / 5000\right)$, a small amount of statistical fluctuation or estimation error in the estimated value will be amplified about $10^{11}$ times. To avoid this, we use the estimated (or equivalent) input quantities which carry the same order of magnitude of statistical fluctuation and weight.

4) There are several reasons that make the logarithmic energy scale the logical choice for the group intervals in the energy axis of phase space. These include:

a) the wide energy $\operatorname{span}(0$ to $1500 \mathrm{eV}$ )

b) the relatively high number density at low energies combined with a low rate and small amount of energy 
exchange due to collision processes. In the highenergy region, the situation is just the opposite.

c) to produce reasonable number of particles in each energy interval [for the reason mentioned in the first section (II-B-I)]. The size and number of intervals are mainly dictated by the range of data (energy range in this case) and number of observations available. Excessive fragmentation of the data may produce many intervals with few occupants. On the other hand, insufficient divisions may obscure important dispersions. Thus an optimum number of intervals will be a compromise between these extremes.

\section{Future Extensions}

The Monte Carlo methods are applicable to the most widely diverse branches of computational mathematics including particle physics (neutron transport for instance), operations research problems such as the investigation of servicing processes, modelling the processes of information transmission, in communication theory, the evaluation of definite integrals, the solution of partial differential equations (e.g. boundry-value problems); the solution of systems of linear equations - inversion of matrices, and many other applications. Problems handled by such methods are in general of two types, the probabilistic and the deterministic, according to whether or not they are directly concerned with the behavior and outcome of random processes. 
Their basis lies in simulating statistical experiments by means of computational techniques. Based on the underlying principles described earlier, various variance reduction techniques can be applied. The present work serves as an example and guide for using the important new techniques such as correlated sampling.

1) The code developed can readily be used to calculate electron energy distribution functions for other gaseous media by merely changing the cross sections input. The model can also be modified to include the electronelectron and electron-ion interactions although this adds more nonlinearities into the system. This would allow calculation for higher source rates and cases where the fractional ionization is large.

2) As for the complex transport problems encountered in areas such as plasmas physics and astrophysics, the model and techniques can readily be extended to the problems with more complex geometry and/or boundry conditions, with inhomogeneous media and multi-dimensions. Also many kinds of nonlinear features can be studied in a realistic fashion.

3) More elaborate error checking and correction procedure (based on the known physical phenomena or theoretical formulations) such as the correction matrix used by Berger (9) should be developed to treat the combined effect of statistical fluctuations and other types of 
uncertainties. This is essential for generating good results for Monte Carlo calculation.

4) More elaborate algorithms or techniques for playing the artificial games of chance by means of weighted cross sections would be a good direction for future research. This must be based on the investigator's intuition and experience, but still it is a promising approach for increasing the efficiency.

5) The present model and techniques can be also implemented on smaller computer systems such as PDP-11 or other similar systems where large amount of fast storage space are not available. The following techniques could be used to process the calculation without storing all the particle data (namely $E_{i}, u_{i}, z_{i}, W t_{i} \forall_{i}$ ). At the beginning of each time cycle, we generate a small number (some reasonable amount, $\simeq 100$ for instance) of particles. The corresponding particle data can be generated from the initial input distribution or from last time cycle. After these particles have been processed, only the accumulated information is stored. A new group of particles come into the system by generating a new set of data, based on the same distributions, which are stored in the same temporary storage space. The previous particle data is destroyed after they have been processed. The same process is repeated until all the necessary prespecified number of particles have been 
processed, and the new distributions are obtained. This eliminates the necessity of storing all the particle data at each time cycle, and this eliminates the large arrays that demand the major amounts of storage space. Due to the random nature of the processing algorithms, and the Markovian behavior of the time cycle process (these time cycle processes are a kind of Markov process), this storage saving algorithm can accomplish the desired results.

\section{E. Concluding Remarks}

In developing any Monte Carlo models and performing Calculations, as mentioned in Chapter $V$ the systematic errors and outright blunders must be detected and removed by testing and comparing with known results.

The various variance reduction techniques are used to increase the relative computation efficiency, reduce inherent random errors or statistical fluctuations. For better calculational results, more samples are favorable even though not economic. In any Monte Carlo calculation there exists a minimum number of samples below which the results or statistics are no longer valid and dependable regardless of the variance reduction schemes employed. In the other words, variance reduction techniques help to reduce statistical fluctuations, but they can not be used to remove all the fluctuations caused by small samples. 
The minimum sample size, is, of course, problem dependent. There are no definite rules or formula for it, thus a cut and try method is necessary until the calculated variance is acceptable.

Monte Carlo calculations are perhaps best in the preliminary stages where they help to give a general idea of the situation and give hints or feelings for trends. If more accurate results are necessary, Monte Carlo calculations may not always be practical good method. In any case, the Monte Carlo method is an important tool in the inferential analysis of the mathematical-physics problems, but it should be viewed as supplementing, rather than replacing, analytic and other numerical methods. 


\section{LIST OF REFERENCES}

1. R. W. L. Thomas and W. R. L. Thomas. "Monte Carlo Simulation of Electrical Discharges in Gases," J. Phys. B (Atom. Molec. Phys.) Ser. 2, Vol. 2, 562, (1969).

2. Tomizo Itoh and Toshimitsu Musha. "Monte Carlo Calculations of Motion of Electrons in Helium," J. Phys. Soc. Japan, Vol. 15, 9, 1675 (1960).

3. J. A. Fleck, Jr. "The Calculation of Nonlinear Radiation Transport by a Monte Carlo Method," Methods in Computational Physics, Vol. 1, 43. New York: Academic Press, (1963).

4. J. A. Fleck, Jr. and J. D. Cummings. "An Implicit Monte Carlo Scheme for Calculating Time and Frequency Dependent Nonlinear Radiation Transport, " UCRL 72924 (1970).

5. G. H. Miley, J. T. Verdeyen, T. Ganley, J. Guyot and P. Thies. "Pumping and Enhancement of Gas Lasers via Ion Beams," Symp. on Electron, Ion \& Laser Beam Technology, University of Colorado, May, 1971.

6. J. C. Guyot, G. H. Miley, J. T. Verdeyen and T. Ganley. "On Gas Laser Pumping Via Nuclear Radiations," Symp. on Research on Uranium Plasmas and Their Technological Applications, University of Florida, 1970.

7. J. C. Guyot, G. H. Miley and J. T. Verdeyen. "Application of a Two-Region Heavy-Charged Particles Model to Noble-gas Plasmas Induced by Nuclear Radiations," Intern. Rept. Nuclear Engineering Program, University of Illinois (1970).

8. L. Herwig. "Summary of Preliminary Studies Concerning Nuclear Pumping of Gas Laser Systems," Report C110.053-5, United Aircraft Corp., East Hartford, Connecticut (1964).

9. M. J. Berger. "Monte Carlo Calculation of the Penetration and Diffusion of Fast Charged Particles," Methods in Computational Physics, Vol. 1, 135-213. New York: Academic Press, 1963.

10. A. E. D. Heylen and T. J. Lewis. "Electron Energy Distribution Functions and Transport Coefficients for the Rare Gases," Proc. R. Soc.A., Vol. 271, 531 (1963). 
11. J. A. Smit. "Berechnung der Geschwindigkeits Verteilung der Elektronen bei Gasentladungen in Helium," Physica, Vol. 3, 543 (1936).

12. J. M. Hammersley and D. C. Handscomb. Monte Carlo Methods. New York: Wiley, 1964.

13. J. M. Hammersley and K. W. Morton. "A New Monte Carlo Technique: Antithetic Variates," Proc. Camb. phil. Soc., Vol. 52,449 (1956).

14. J. M. Hammersley and J. G. Mauldon. "General Principles of Antithetic Variates," Proc. Camb. phil. Soc., Vol. 52, 476 (1956).

15. Y. A. Schreider. The Monte Carlo Method. London: Pergamon Press, 1966.

16. E. D. Cashwell and C. J. Everett. A Practical Manual on the Monte Carlo Method for Random Walk Problems. New York: Pergamon Press, 1959.

17. M. A. Uman. Introduction to Plasma Physics. New York: McGraw Hili, 1964.

18. I. G. Parratt. Probability and Experimental Errors in Science. New York: Wiley, 1961.

19. T. D. Sterling and S. V. Pollack. Introduction to Statistical Data Processing. New Jersey: PrenticeHall, 1968.

20. S. Karlin and W. J. Studden. Tchebycheff Systems: With Applications in Analysis and Statistics. New York: Wiley, 1966.

21. L. L. House and L. W. Avery. "The Monte Carlo Technique Applied to Radiative Transfer," J. Quant. Spectrosc. Radiat. Transfer, Vol. 9, 1579 (1969).

22. H. Kahn. "Use of Different Monte Carlo Sampling Techniques," Symposium on Monte Carlo Methods. New York: Wiley, 1956 .

23. R. J. Cicerone and S. A. Bowhill. "Monte Carlo and Thomson-scatter plasma-line studies of Ionospheric Photoelectrons," Aeronomy Rept., No. 39, University of Illinois, Urbana, Illinois (1970). 
24. A. Nordsieck and B. L. Hicks. "Monte Carlo Evaluation of the Boltzmann Collision Integral," CSL Report R-307, University of Illinois (1966).

25. S. M. Yen and B. L. Hick. "On the Accuracy of Approximate Solutions of the Boltzmann Equation," CSL Report R-308, University of Illinois, Urbana, Illinois (1966).

26. M. Y. Youakim, C. H. Liu and K. C. Yeh. "Generation of Random Hypersurfaces," Intern. Rept., Ionoshere Radio Laboratory, University of Illinois, Urbana, Illinois (1970).

27. M. Rich and S. S. Blackman. "A Method for Eulerian Fluid Dynamics," LAMS-2826, UC-32, Mathematics and Computers (1963).

28. W. D. Little. "Hybrid Computer Solutions of Partial Differential Equations by Monte Carlo Methods," Proc. FJCC, 181 (1966).

29. L. B. Miller and G. H. Miley. "Some Monte Carlo Techniques for the Calculation of Doppler Coefficients," Nuclear Science and Engineering, Vol. 40, 438 (1970).

30. C. K. Birdsall and D. Fuss. "Cloud-In-Cell Computer Experiments in Two and Three Dimensions," Proc. APS Topical Conference on Numerical Simulation of Plasma. Los Alamos Scientific Laboratory, University of California (1968).

31. M. Goodrich. "Electron Scattering in Helium," Physical Review, Vol. 54, 259 (1937).

32. G. H. Miley and R. H. Lo. "Calculation of Electron Energy Flux Distribution in Noble Gases," To be published in Proc. 18th Annual Meeting of ANS, June, 1972.

33. R. H. Lo. Department of Nuclear Engineering, University of Illinois, Private Communication, April, 1972.

34. W. Jesse and J. Sadouski. "Ionization in Pure Gas and the Average Energy to Make an Ion Pair for Alpha and Beta Particles," Phys. Rev., Vol. 97, 1665 (1955).

35. S. C. Brown. Basic Data of Plasma Physics. Cambridge, Massachusetts: The M.I.T. Press, 1966. 
36. T. Holstein. "Energy Distribution of Electrons in High Frequency Gas Discharges," Phys. Rev. Vol. 70, $5,367(1946)$.

37. J. T. Ganley. Effect of a Heavy Particle External Ionization Source on $\mathrm{CO}_{2}$ Laser Discharges, Ph.D. Thesis, University of IIlinois, 1972. 
APPENDIX






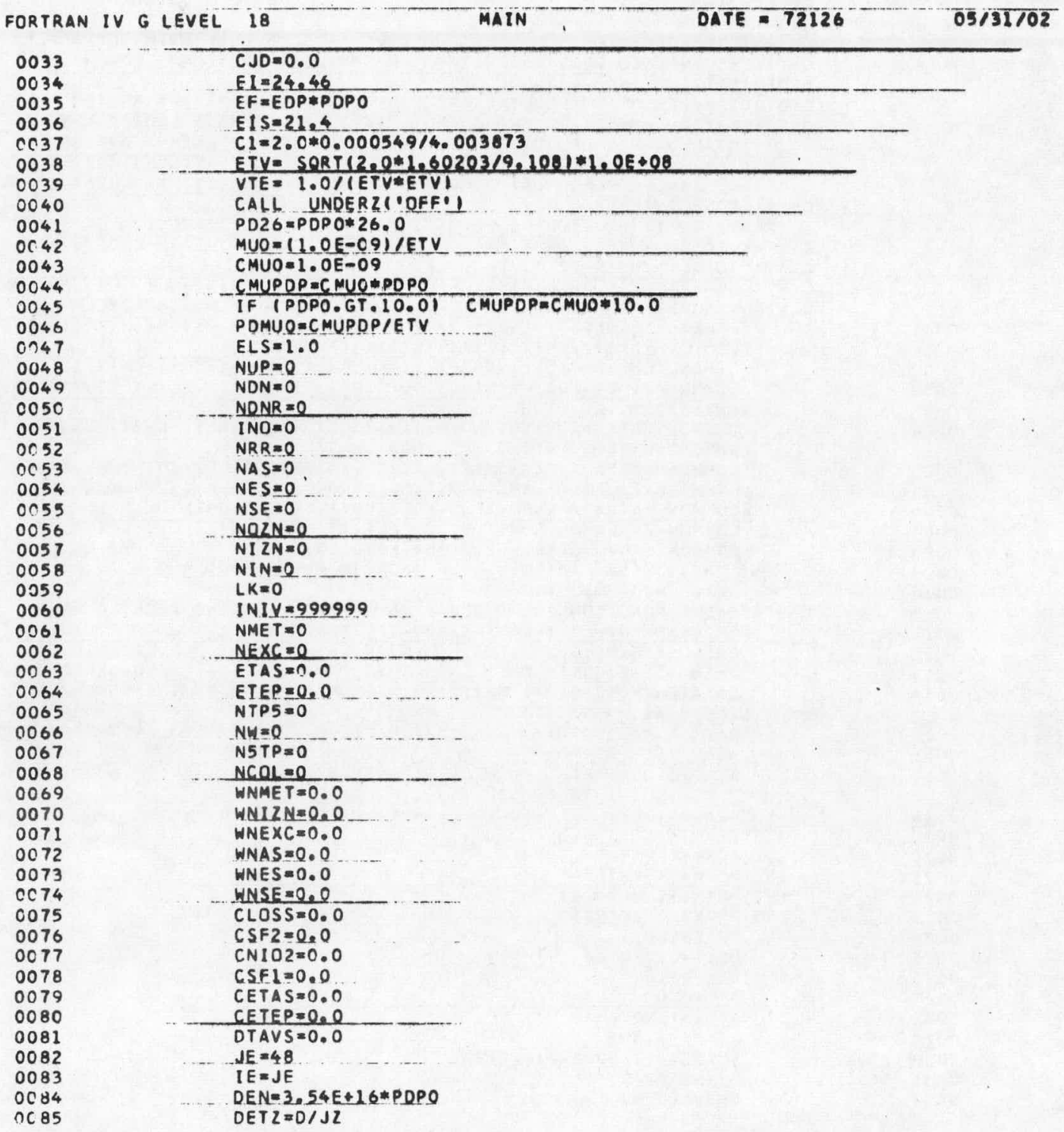









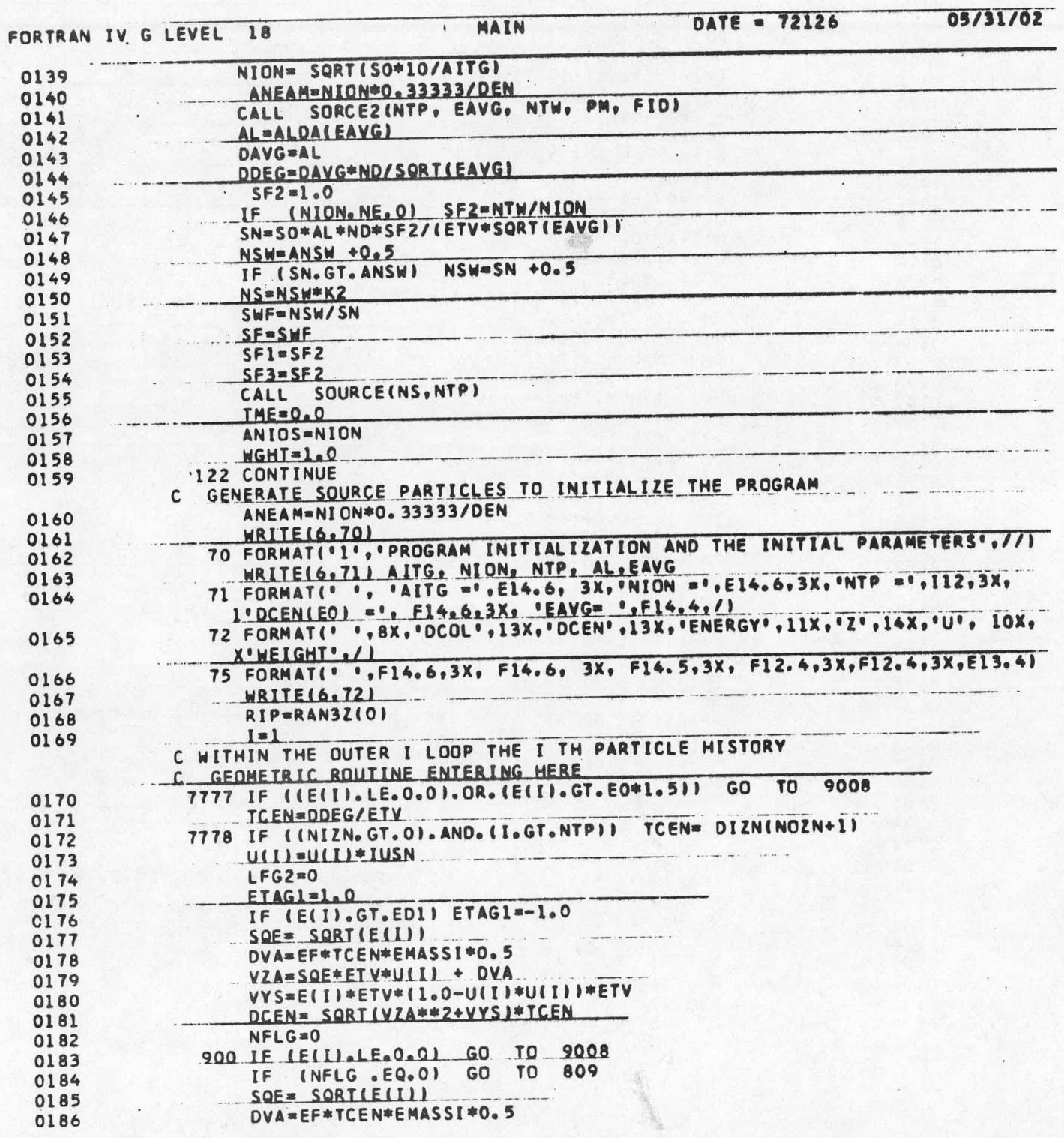




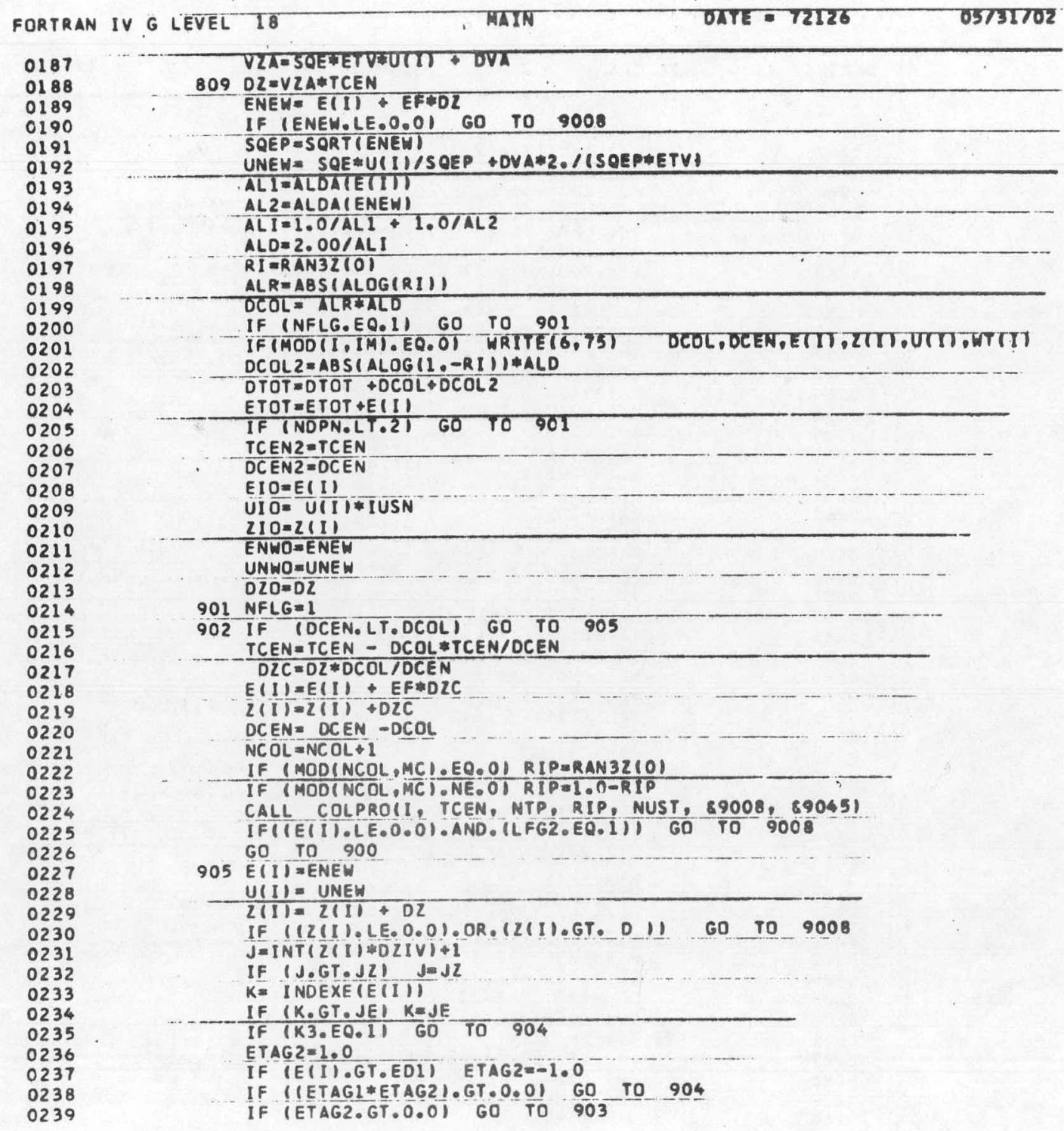




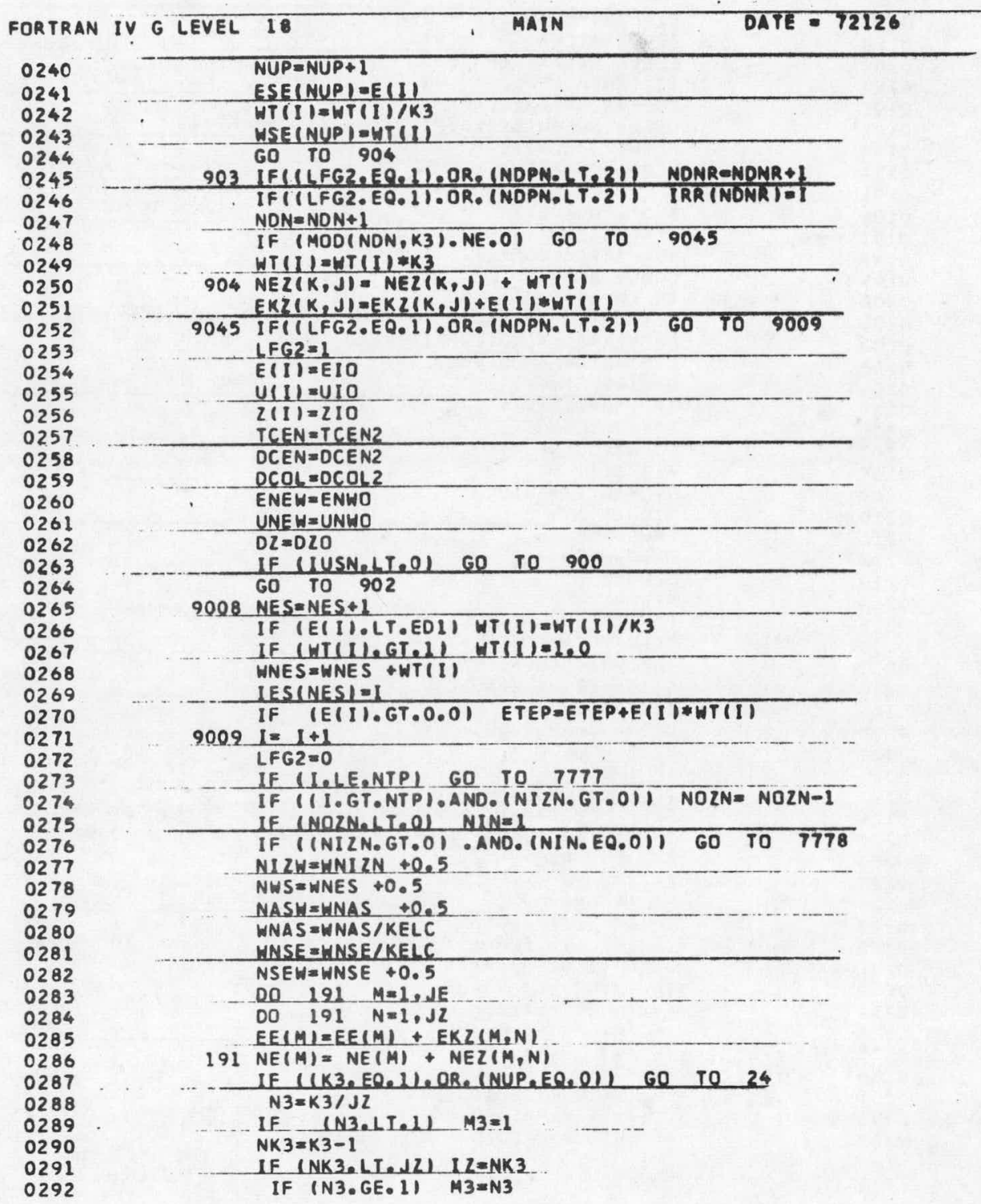

$05 / 31 / 08$ 


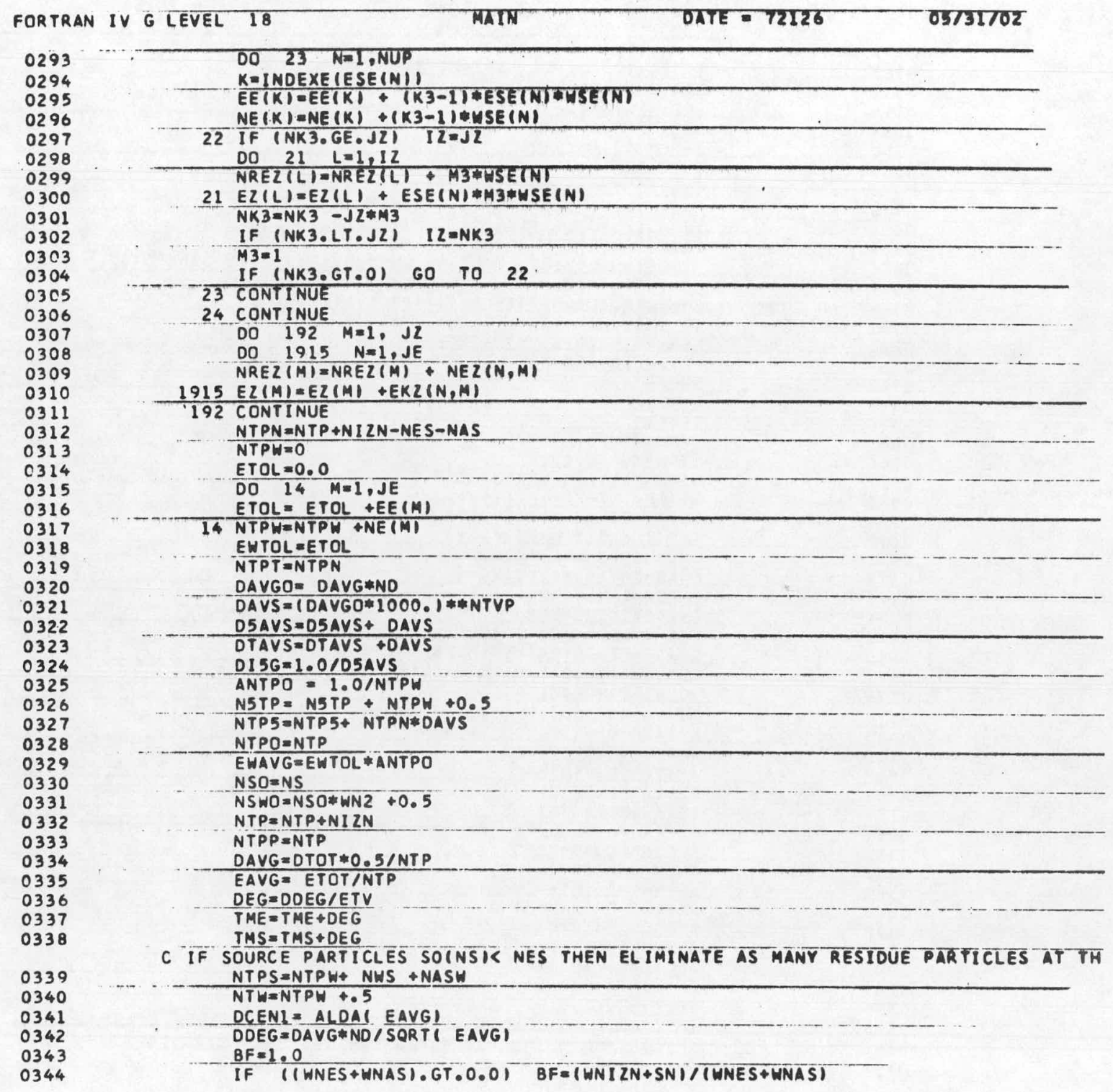




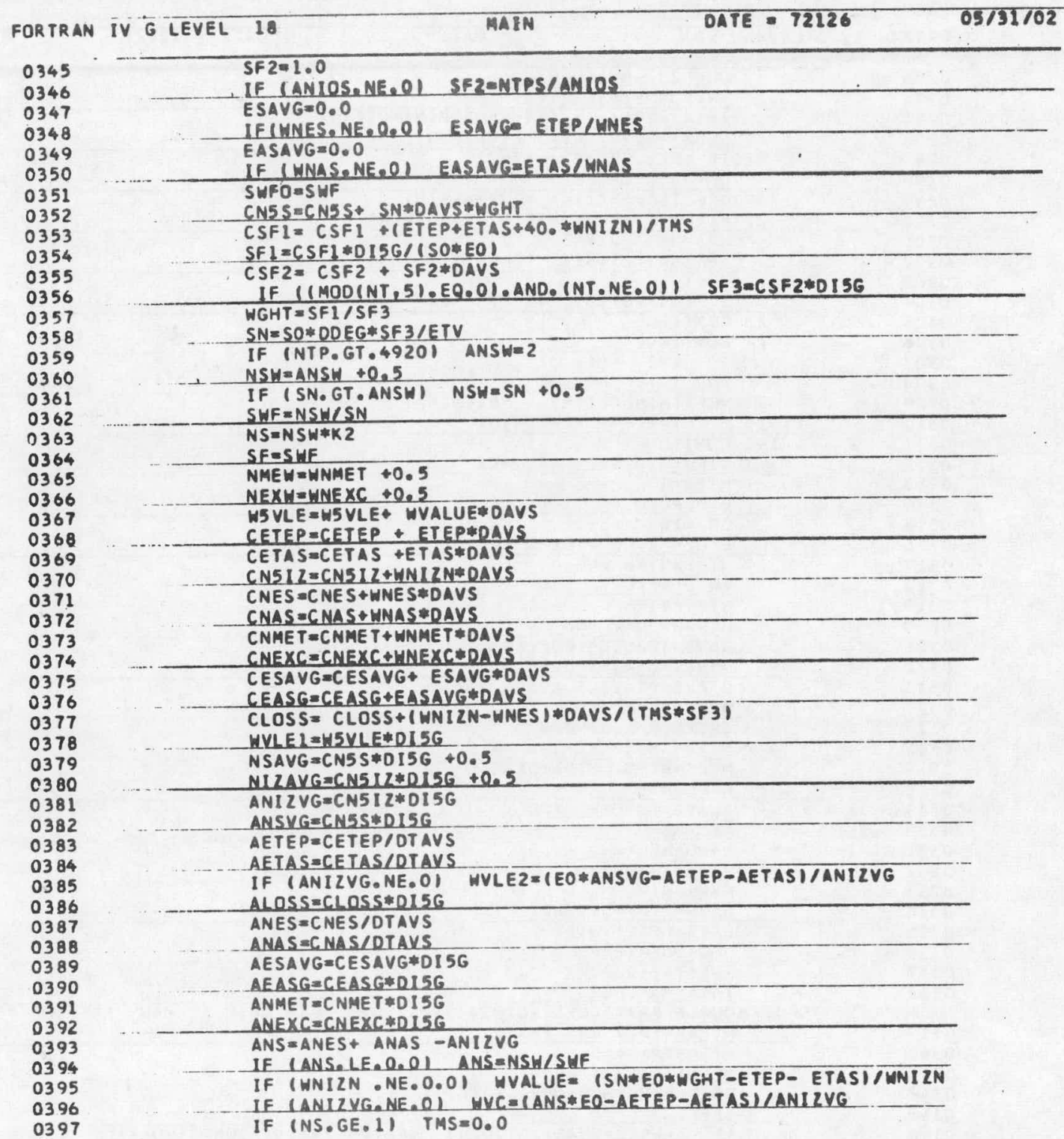




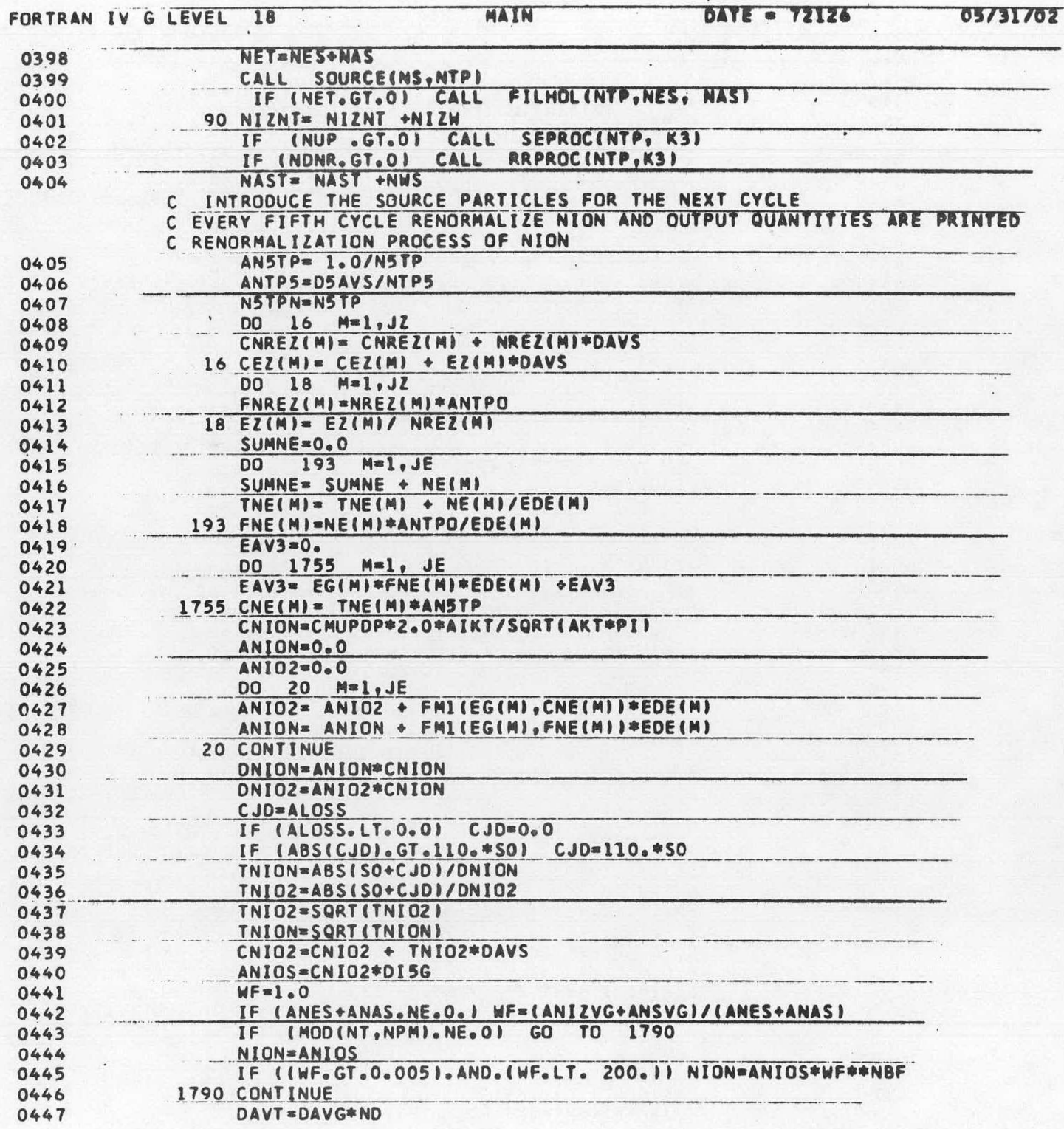




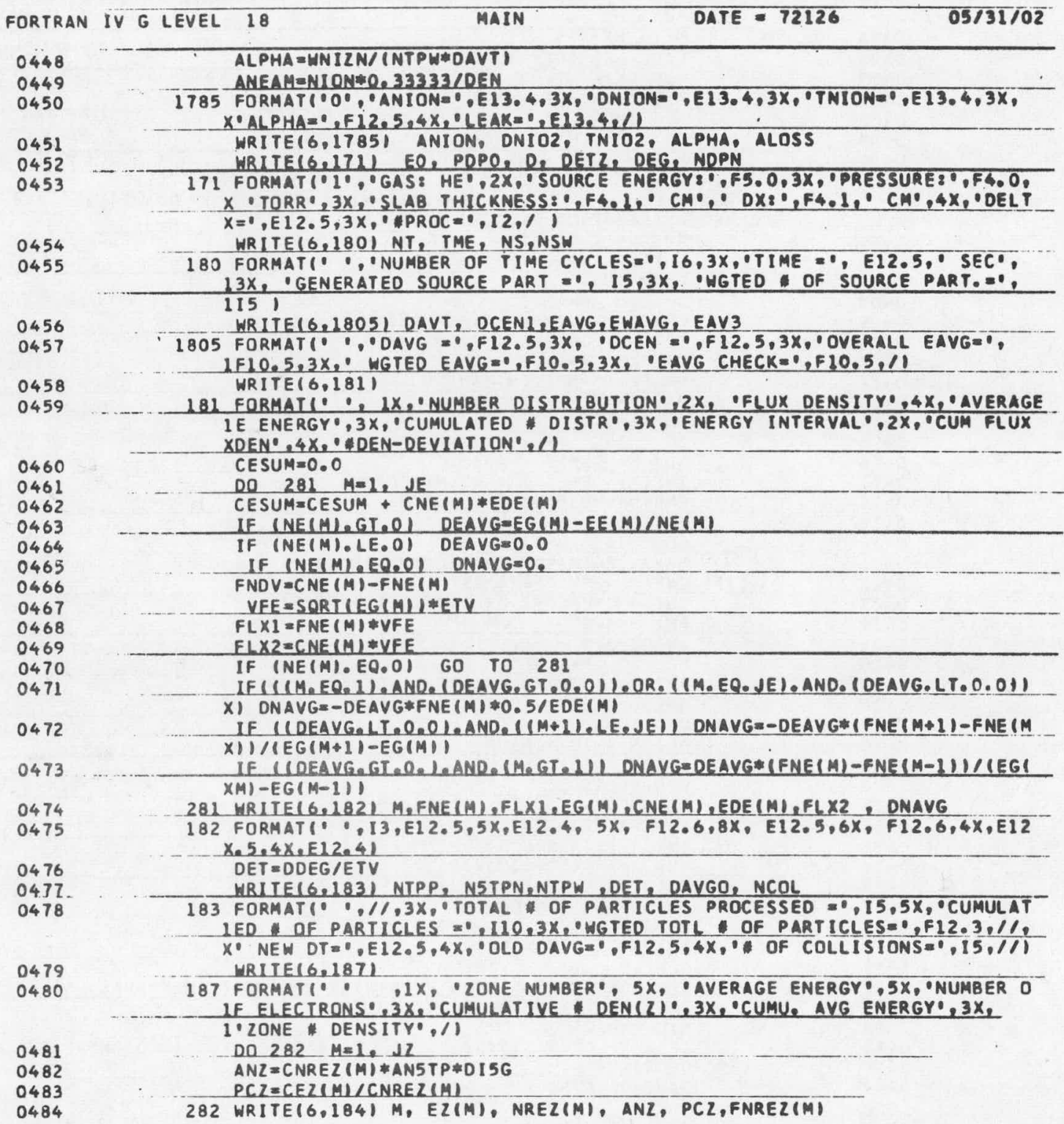




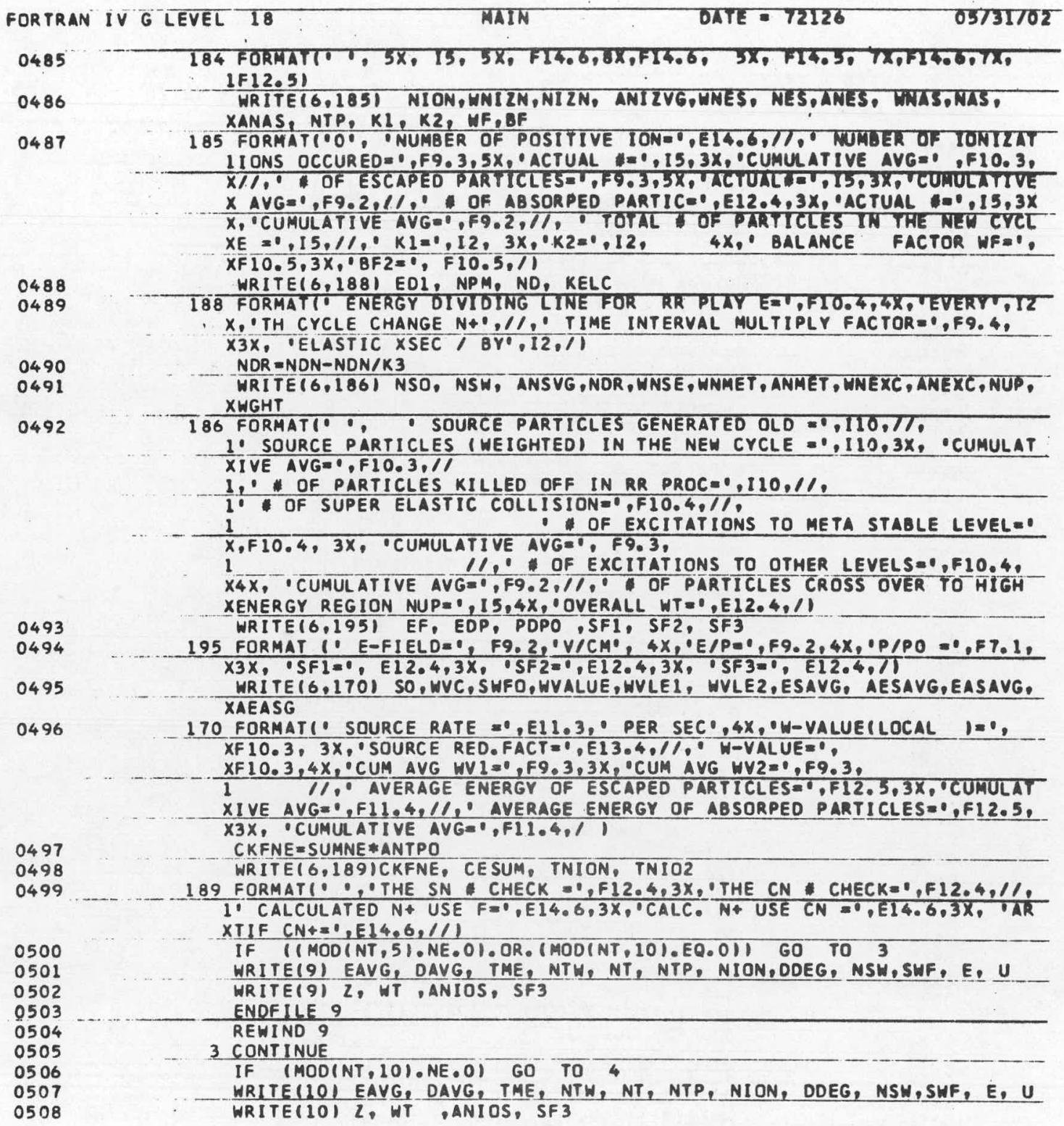




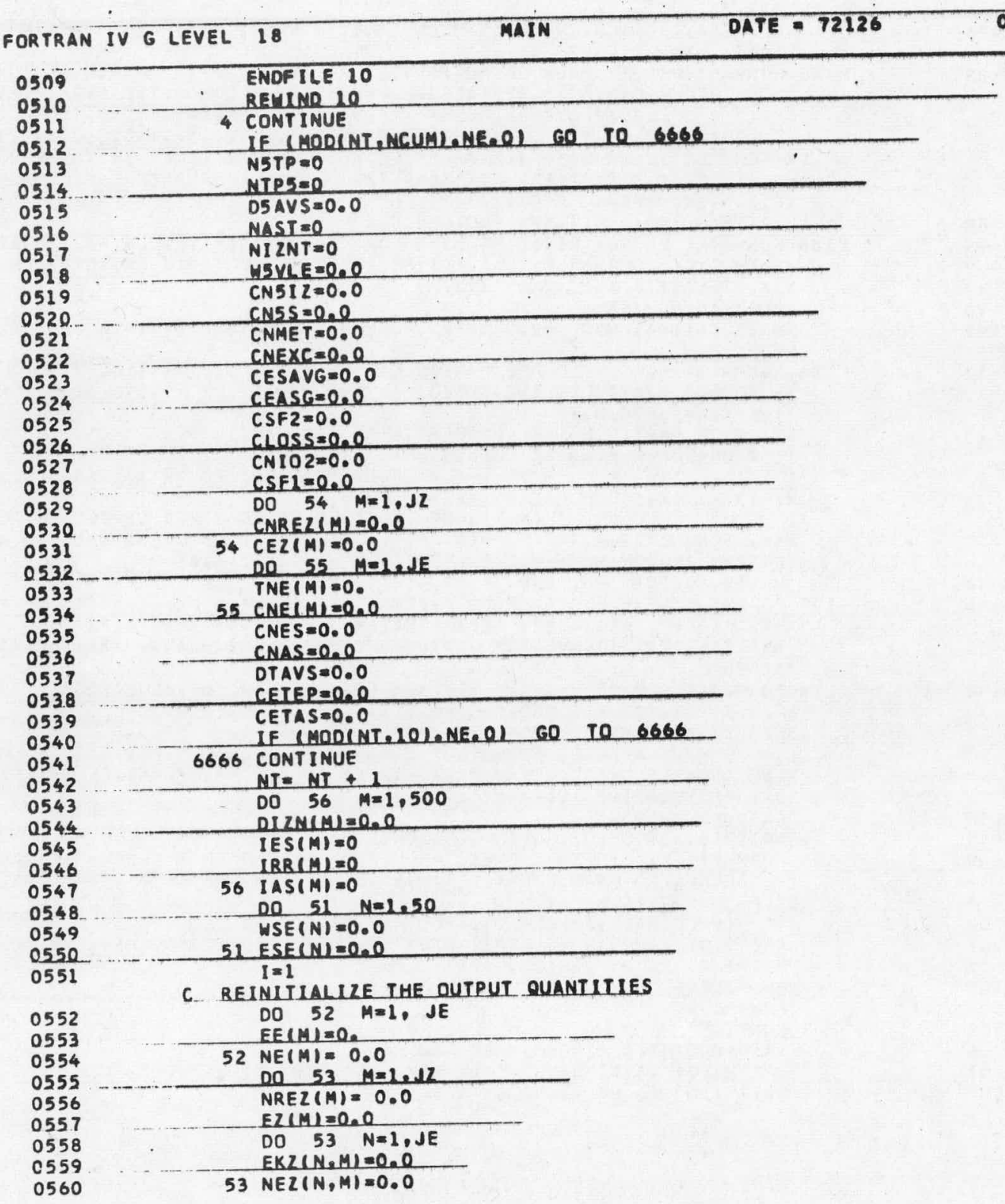




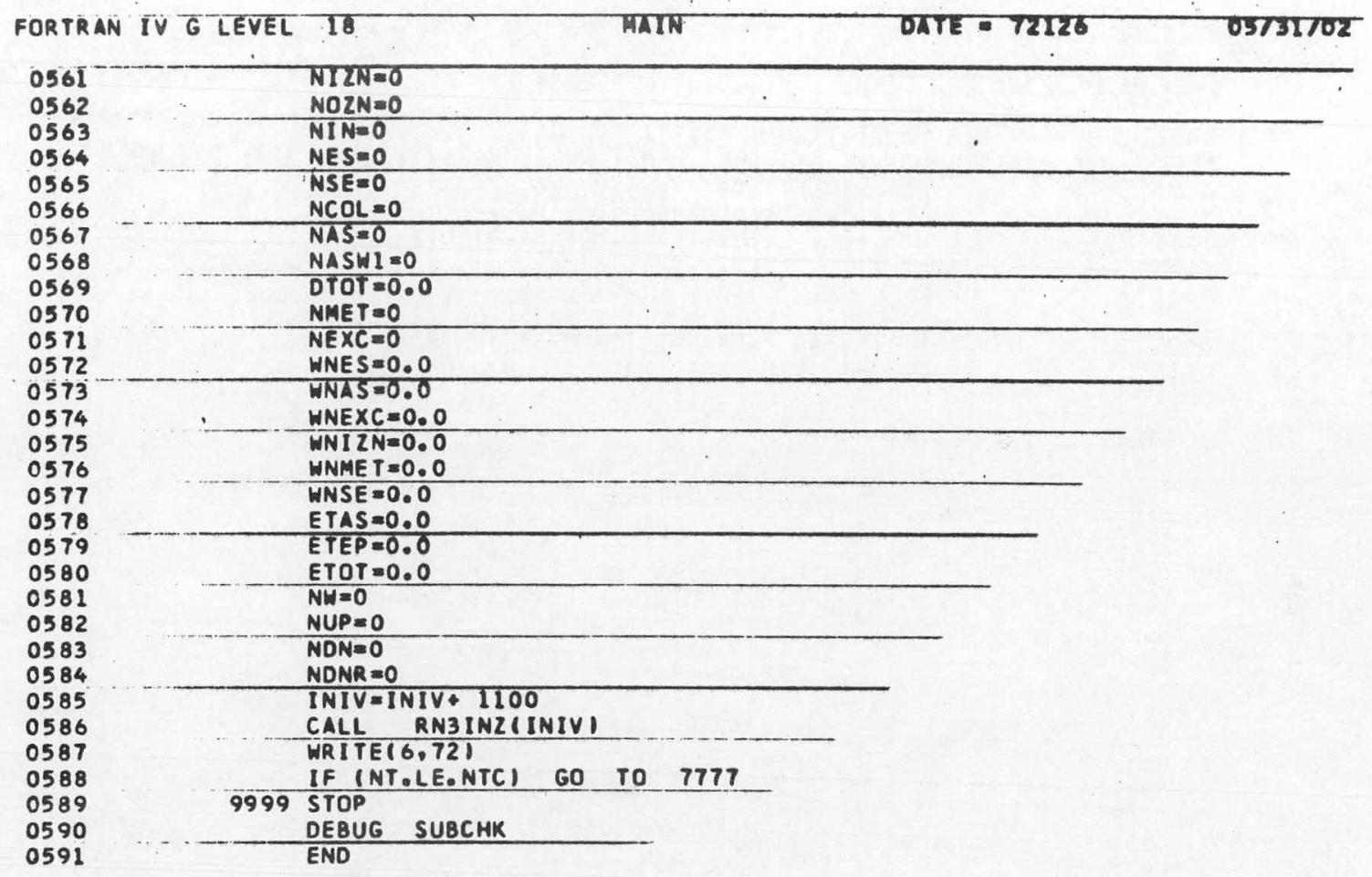




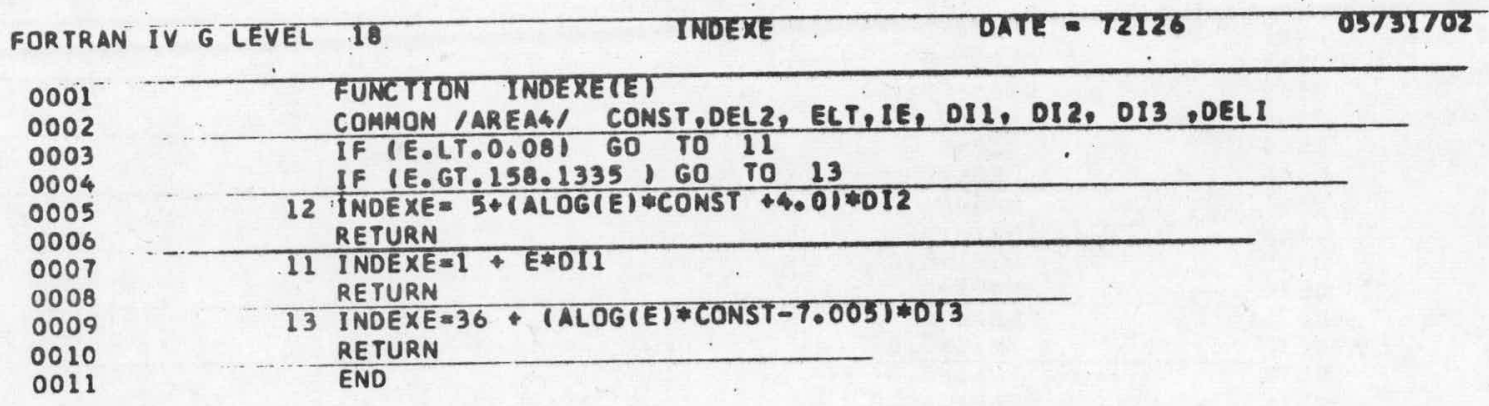




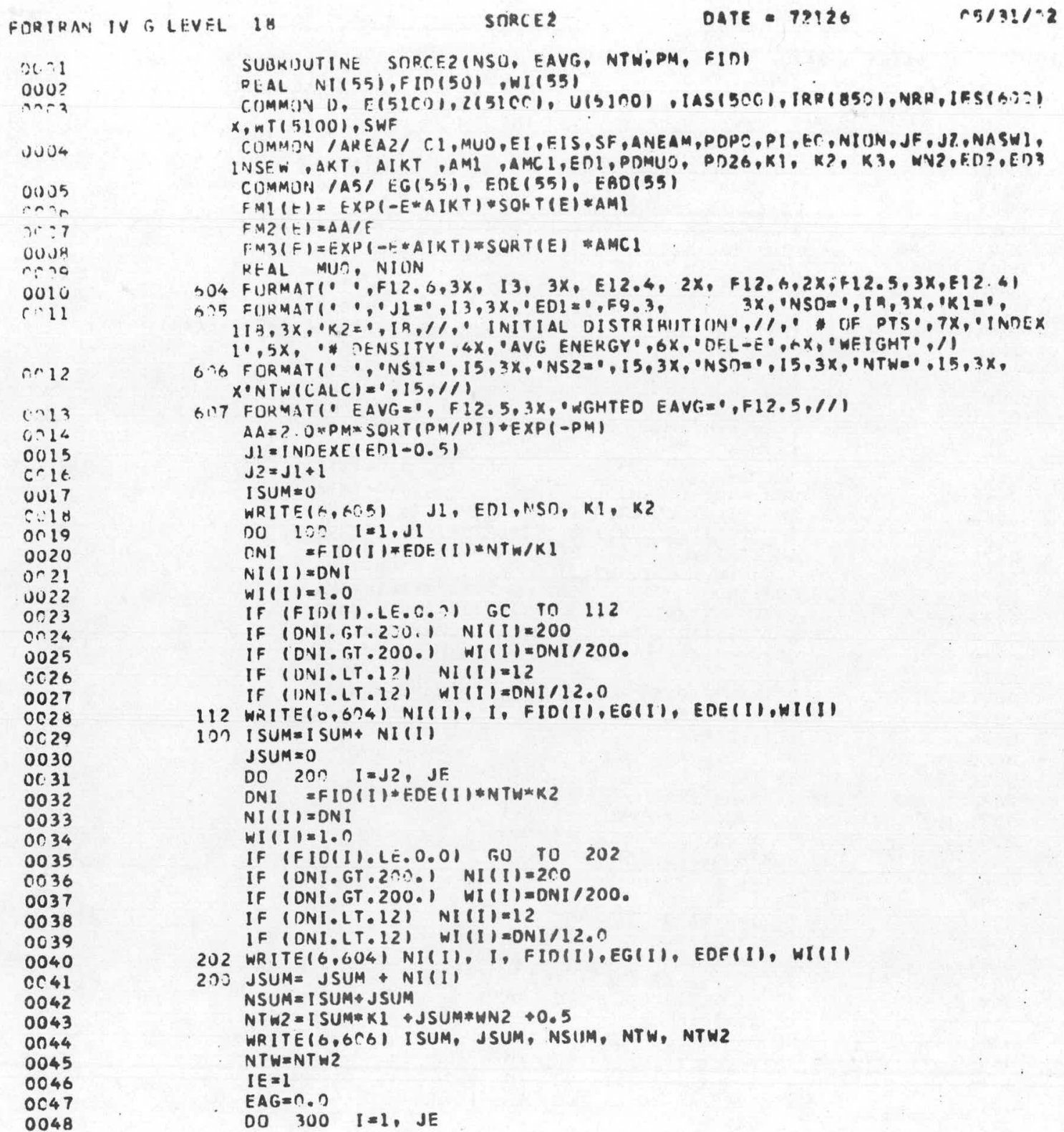









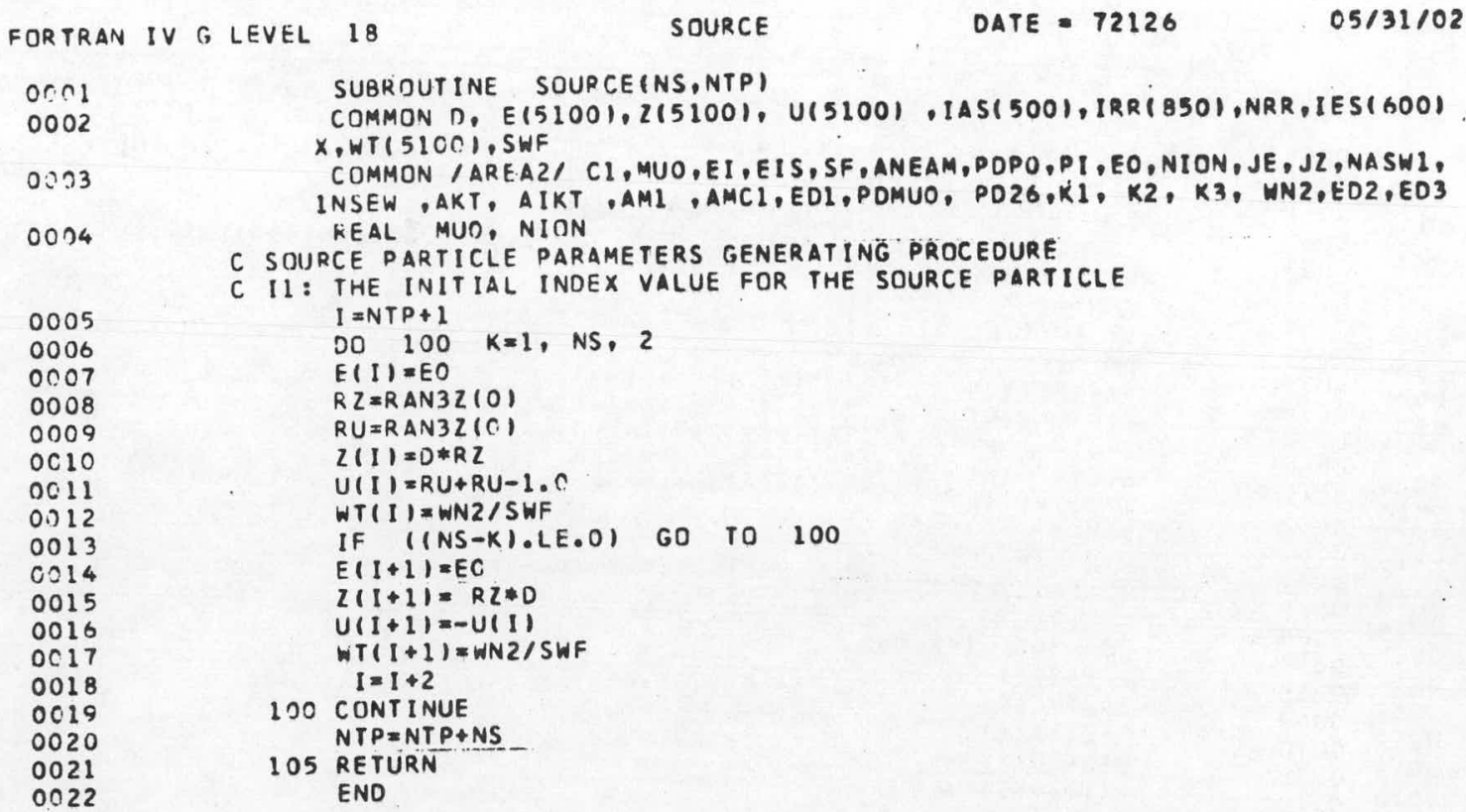

0022 







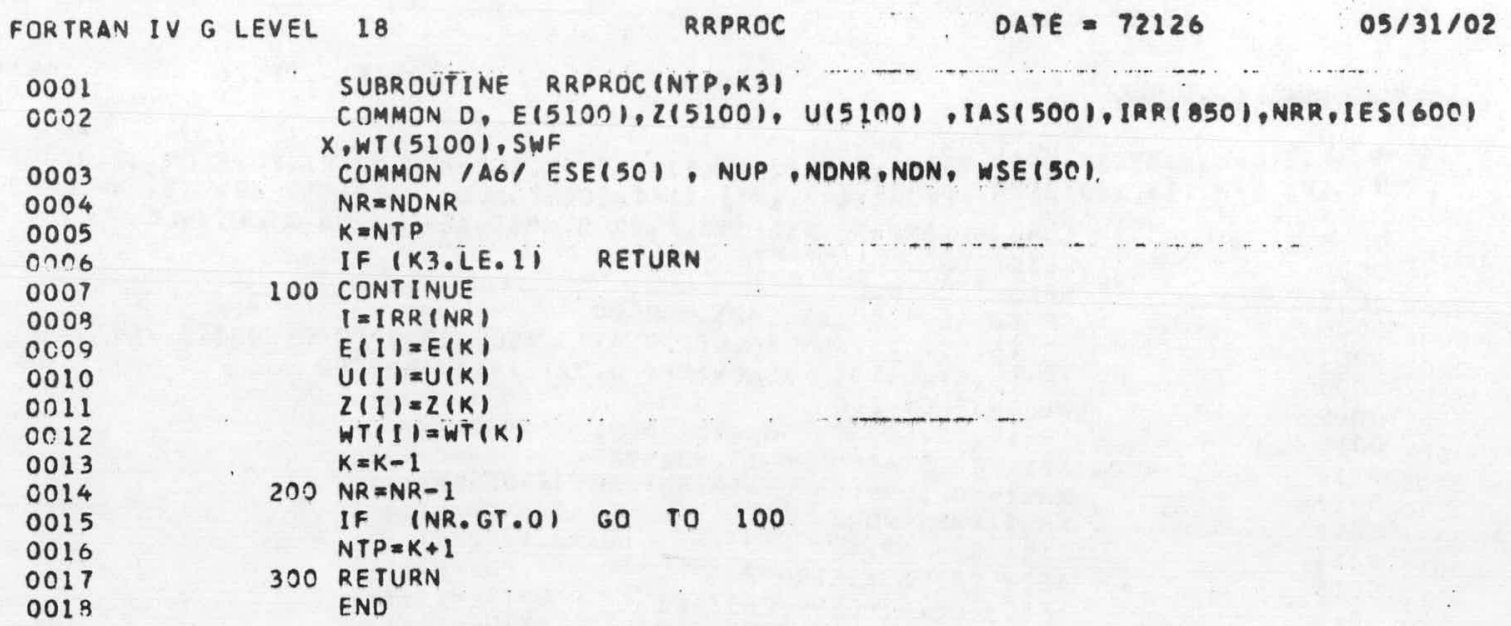


144

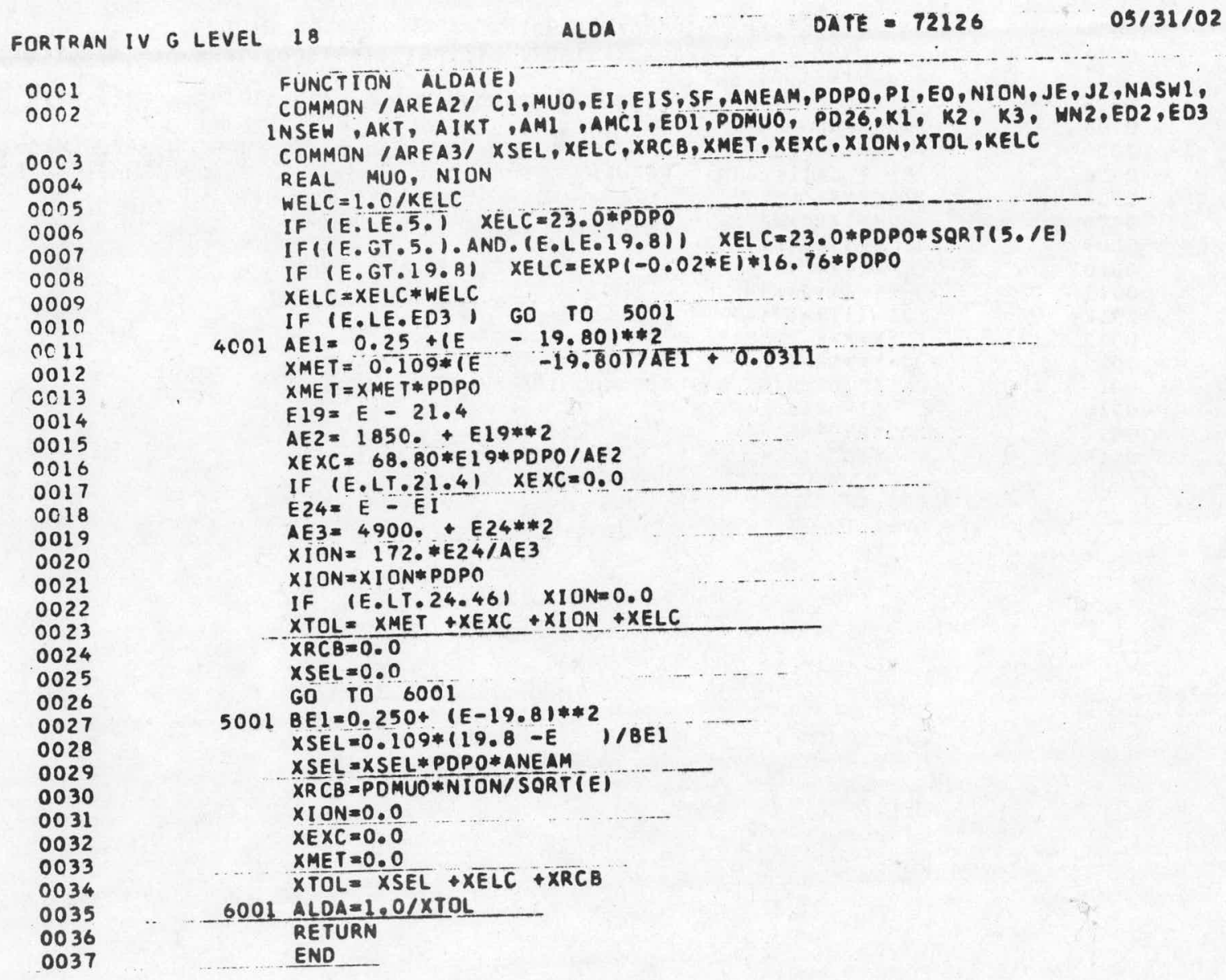




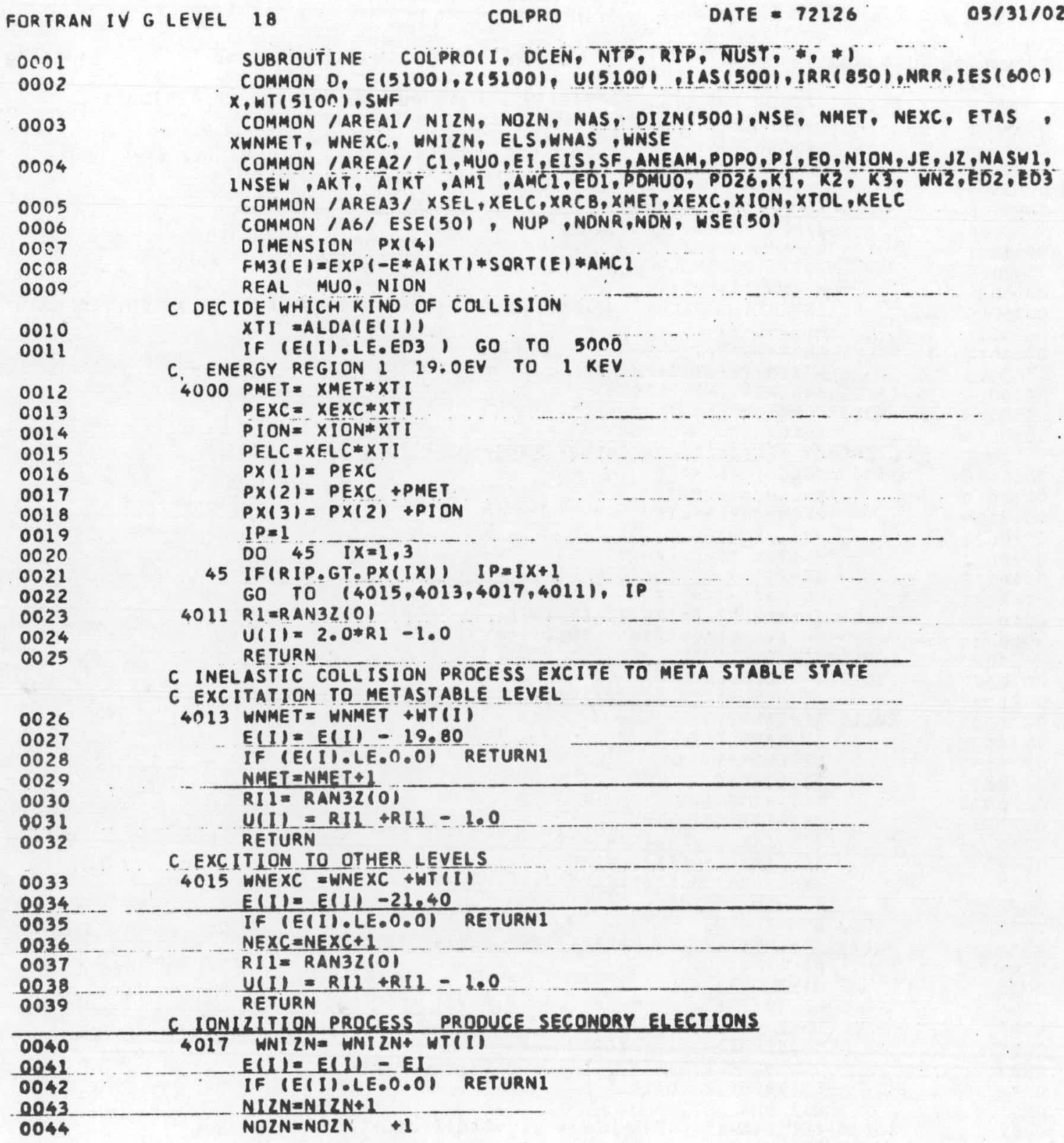




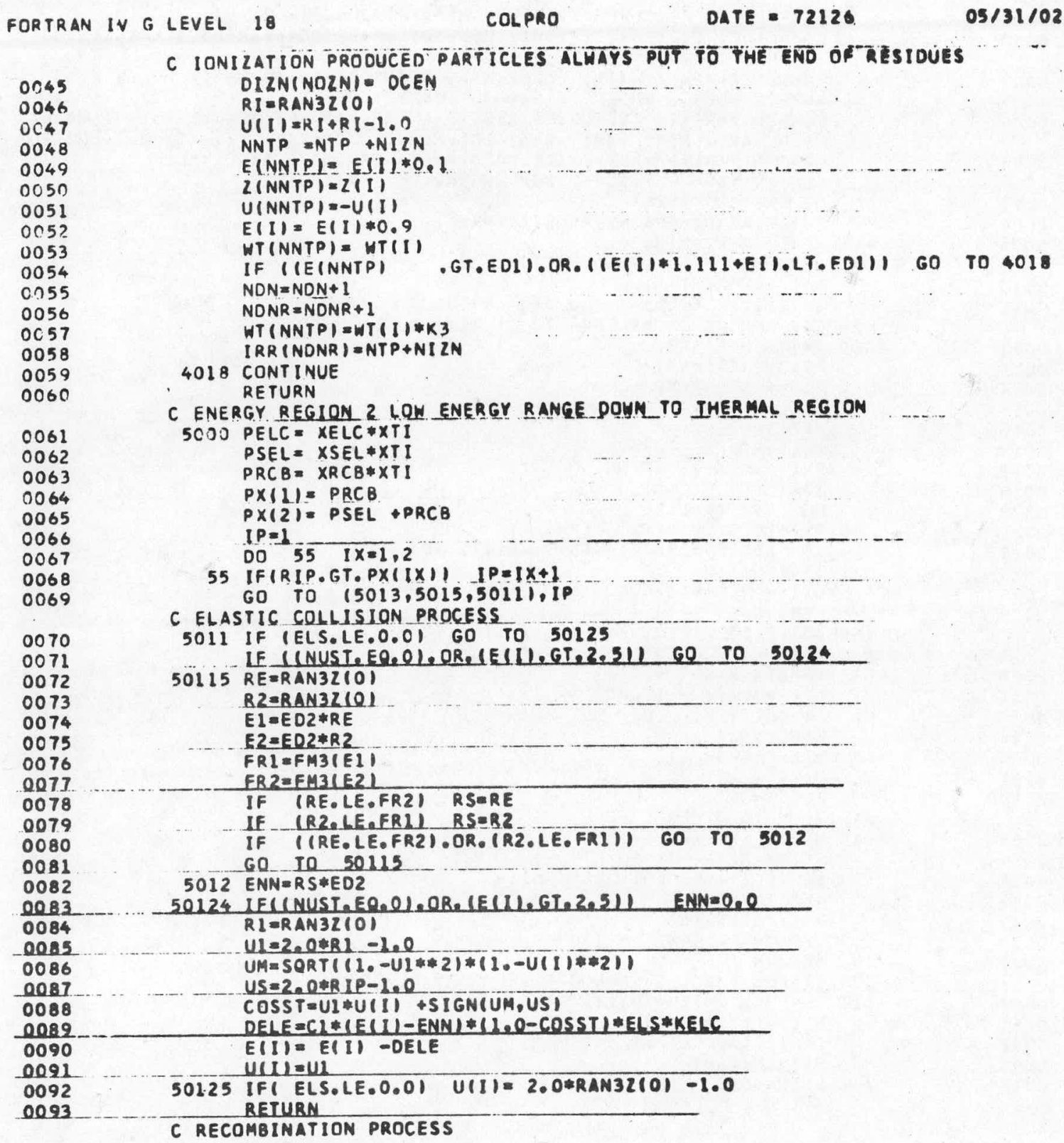




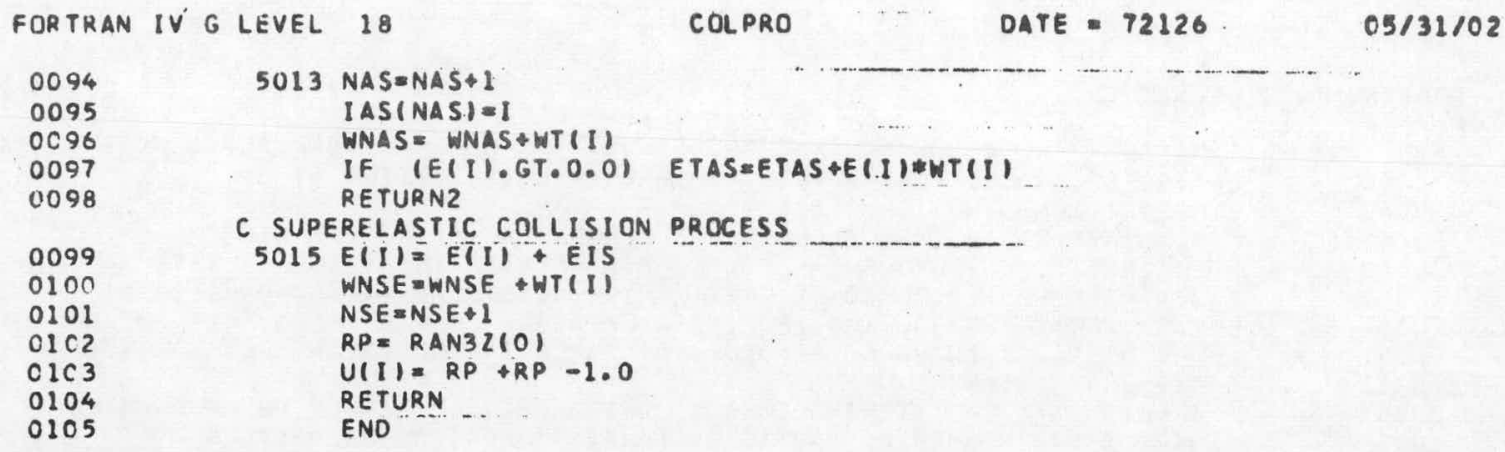




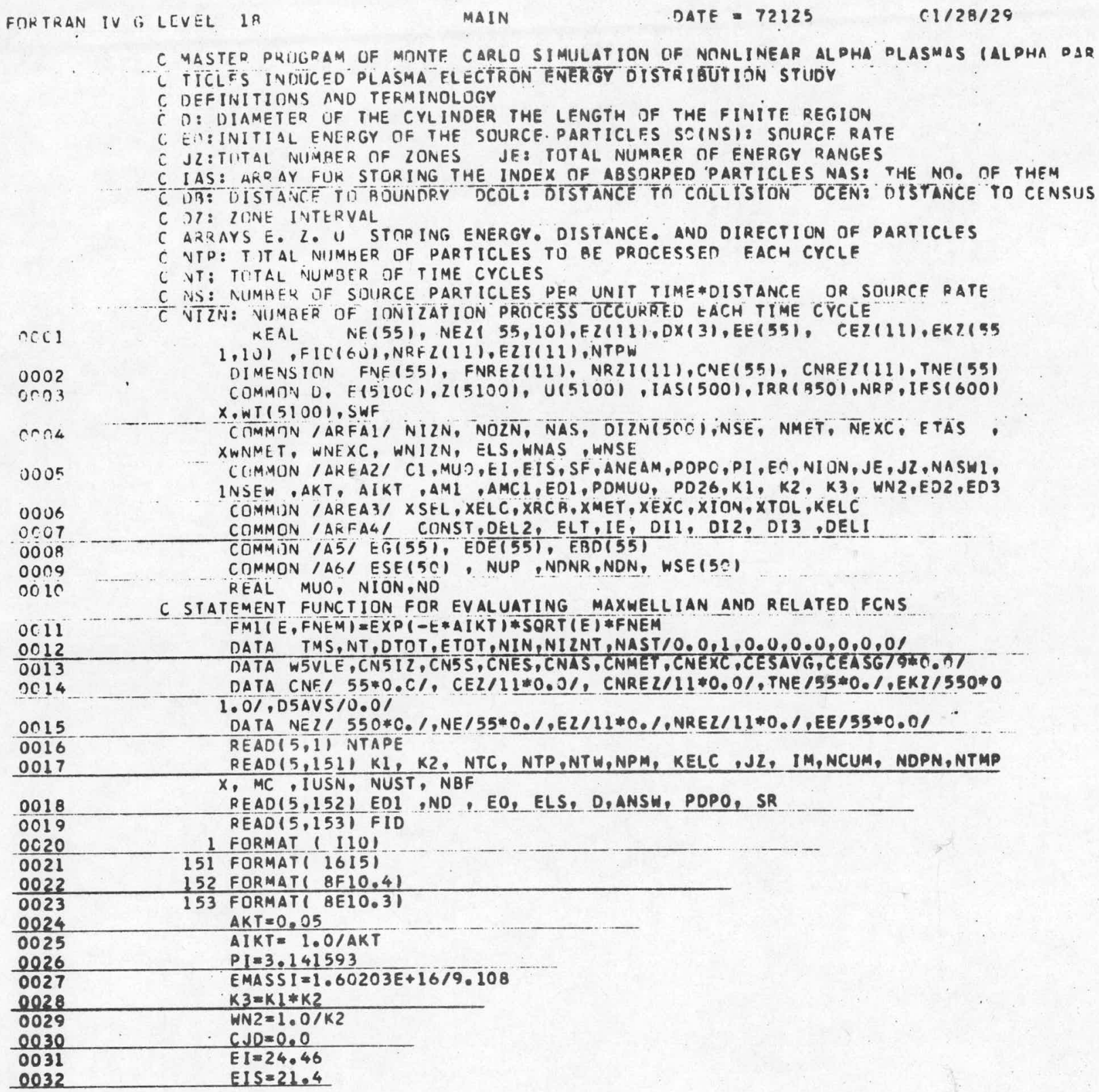




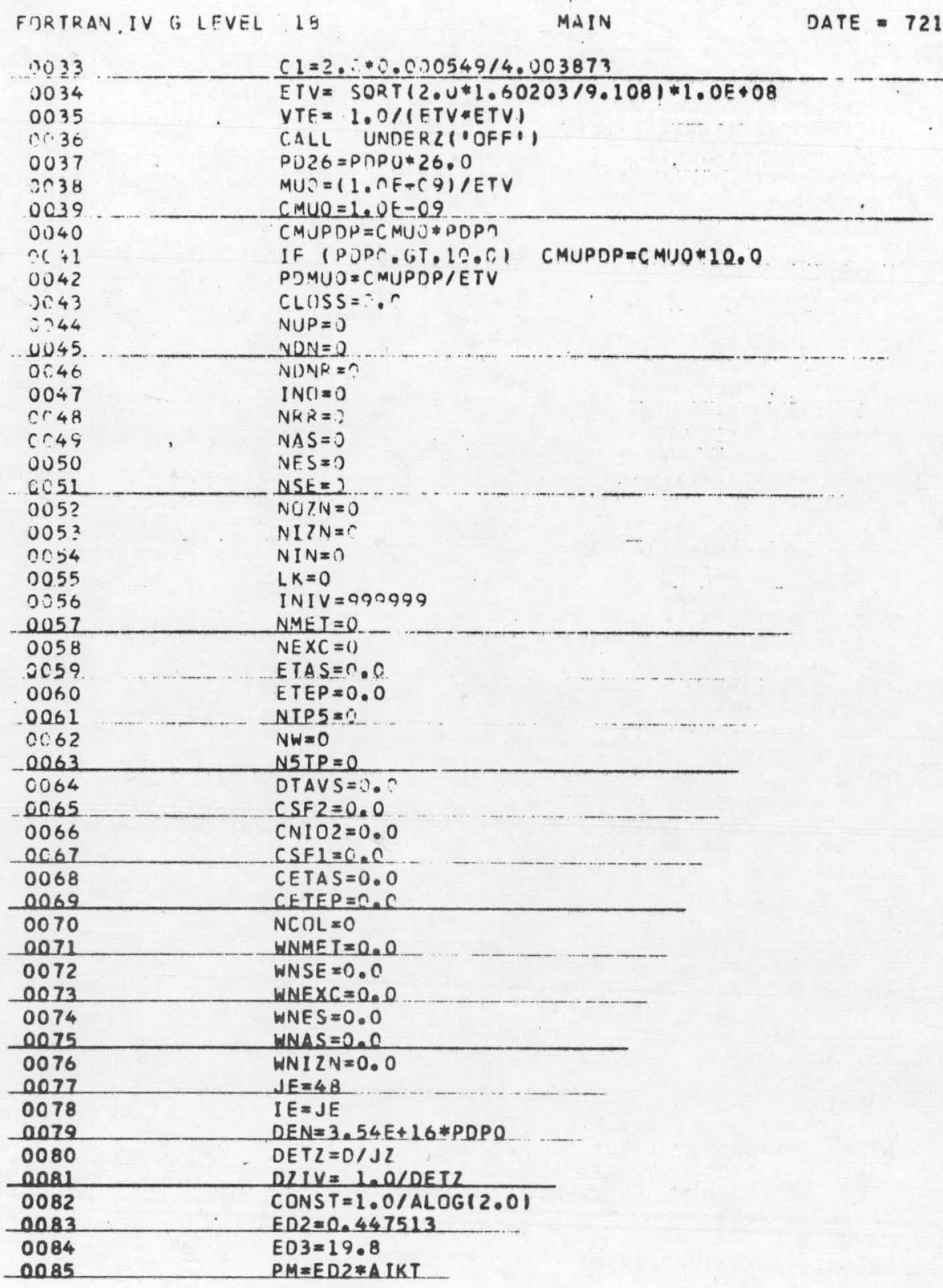




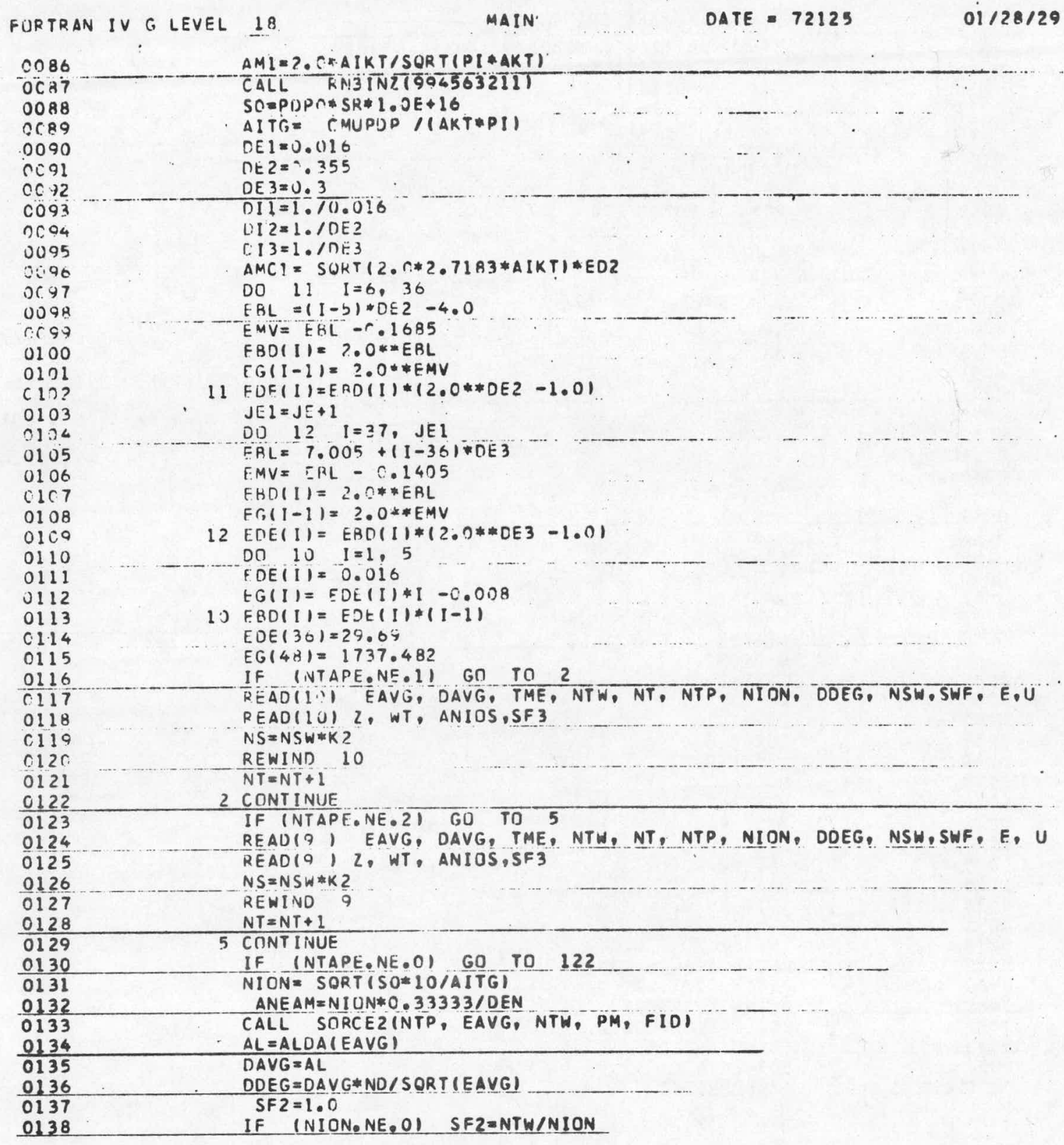









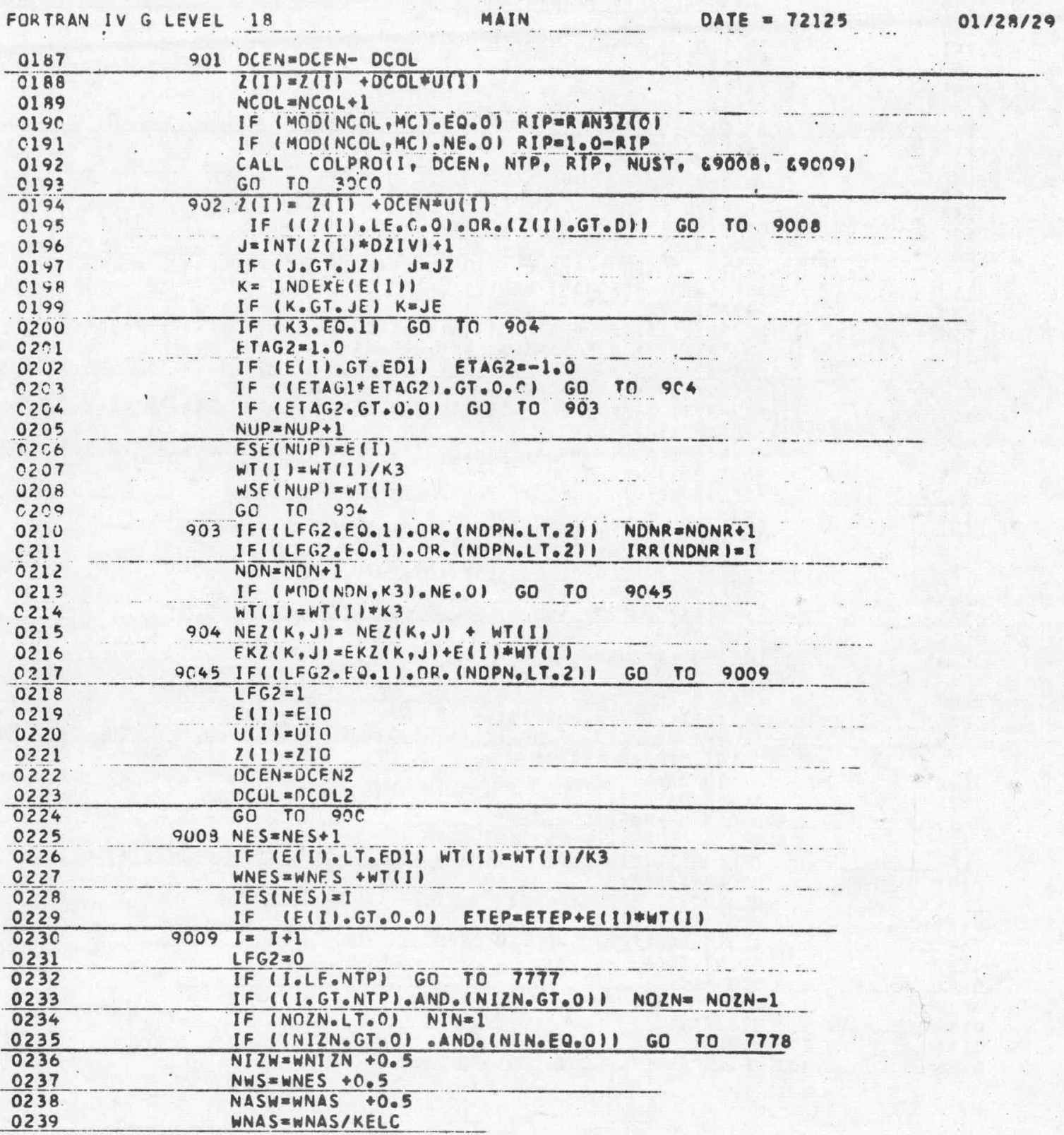


FORTRAN IV G LEVEL 18

MAIN

DATE $=72125$

$01 / 28 / 29$

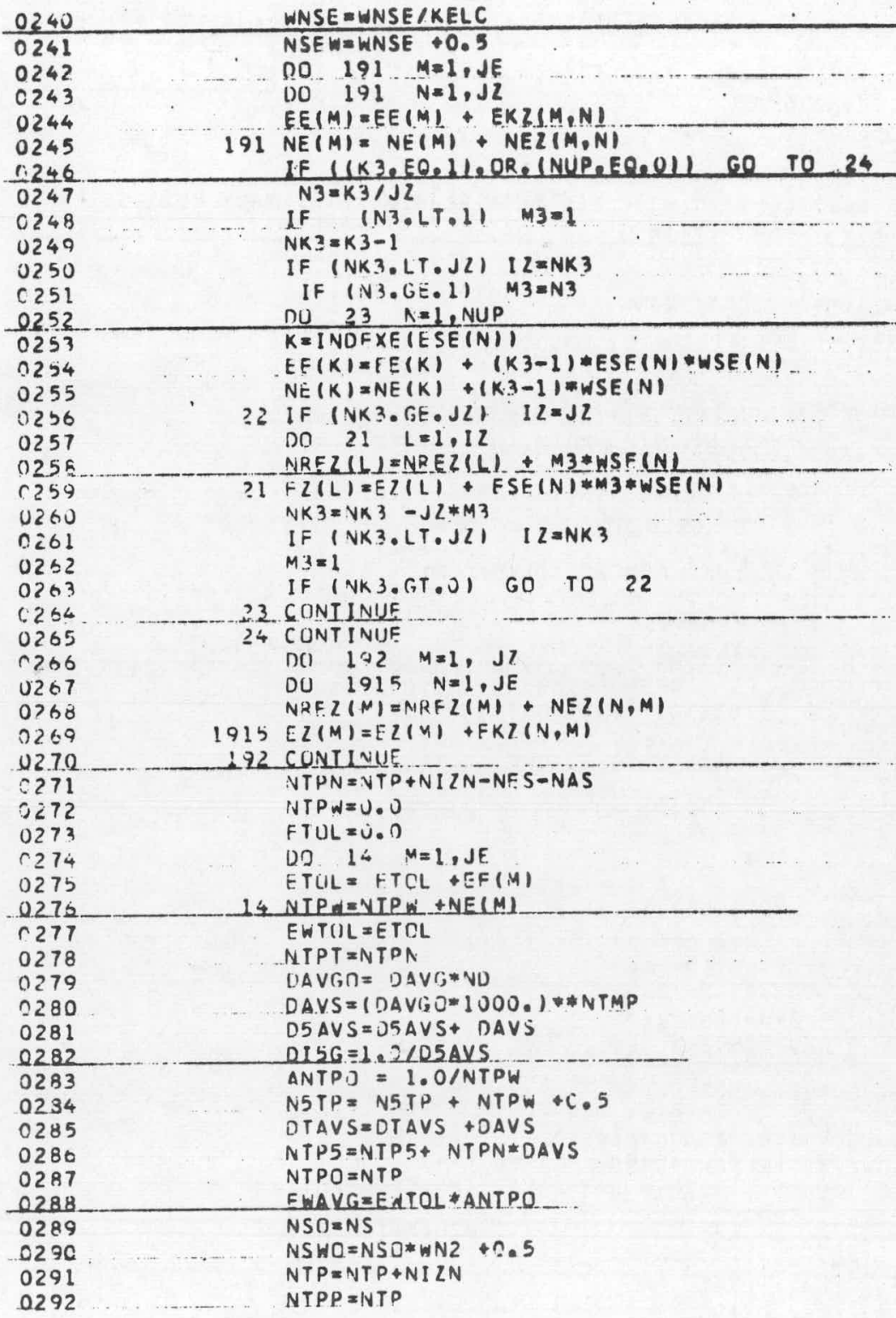




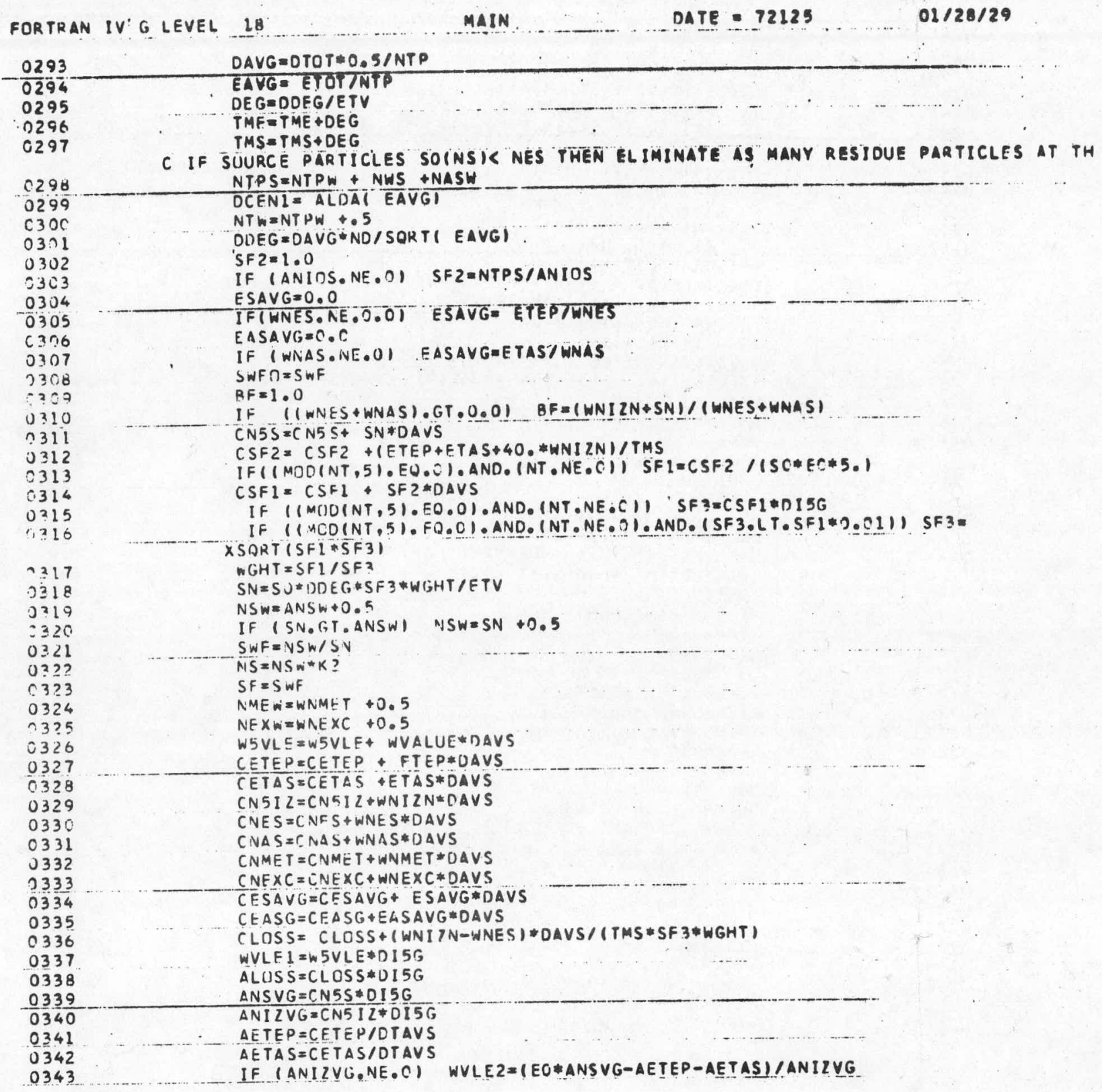


FORTRAN :IY..G.LEYEL IO

DAIE $=.72125$

$02 / 20 / 29$

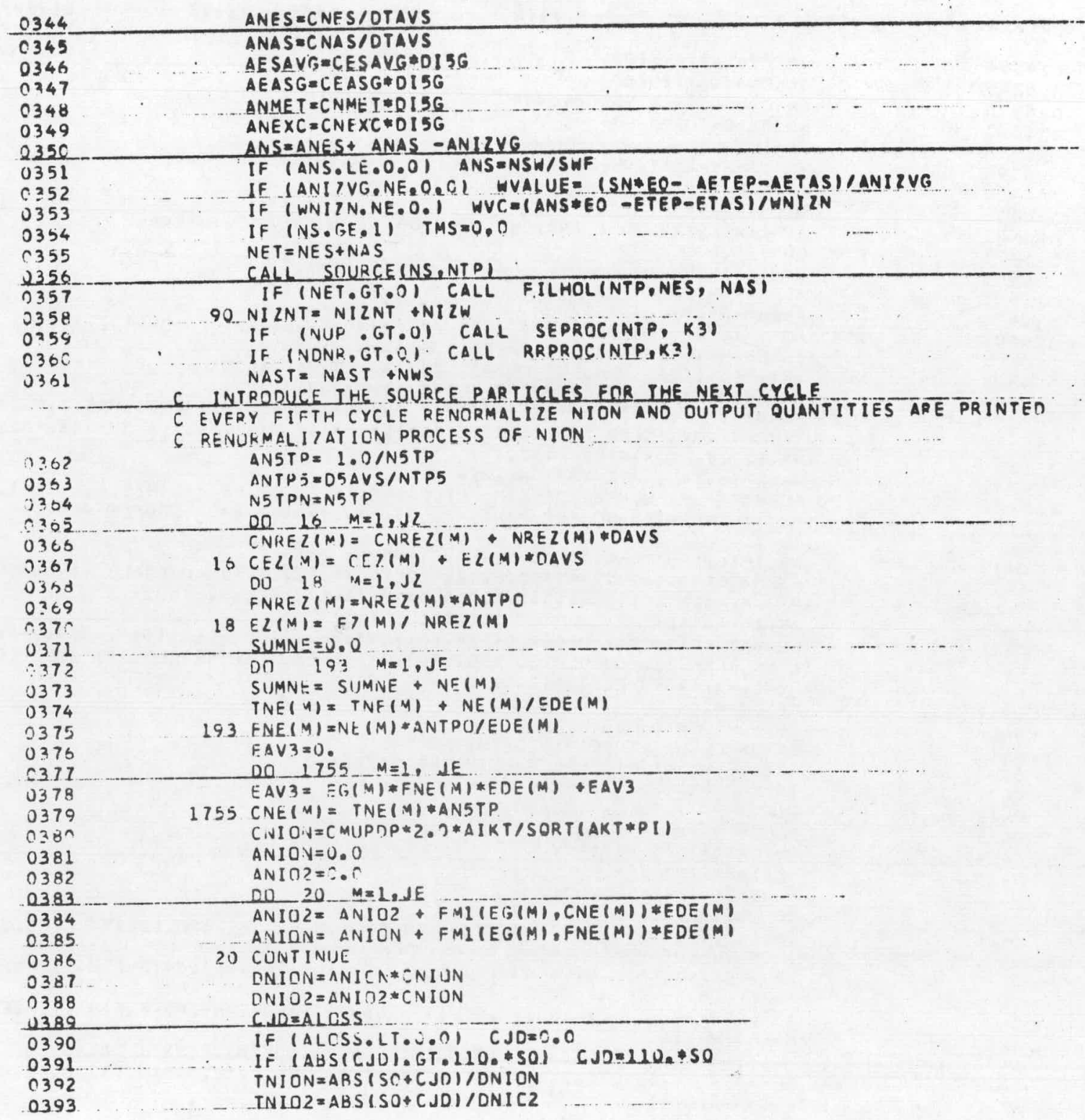




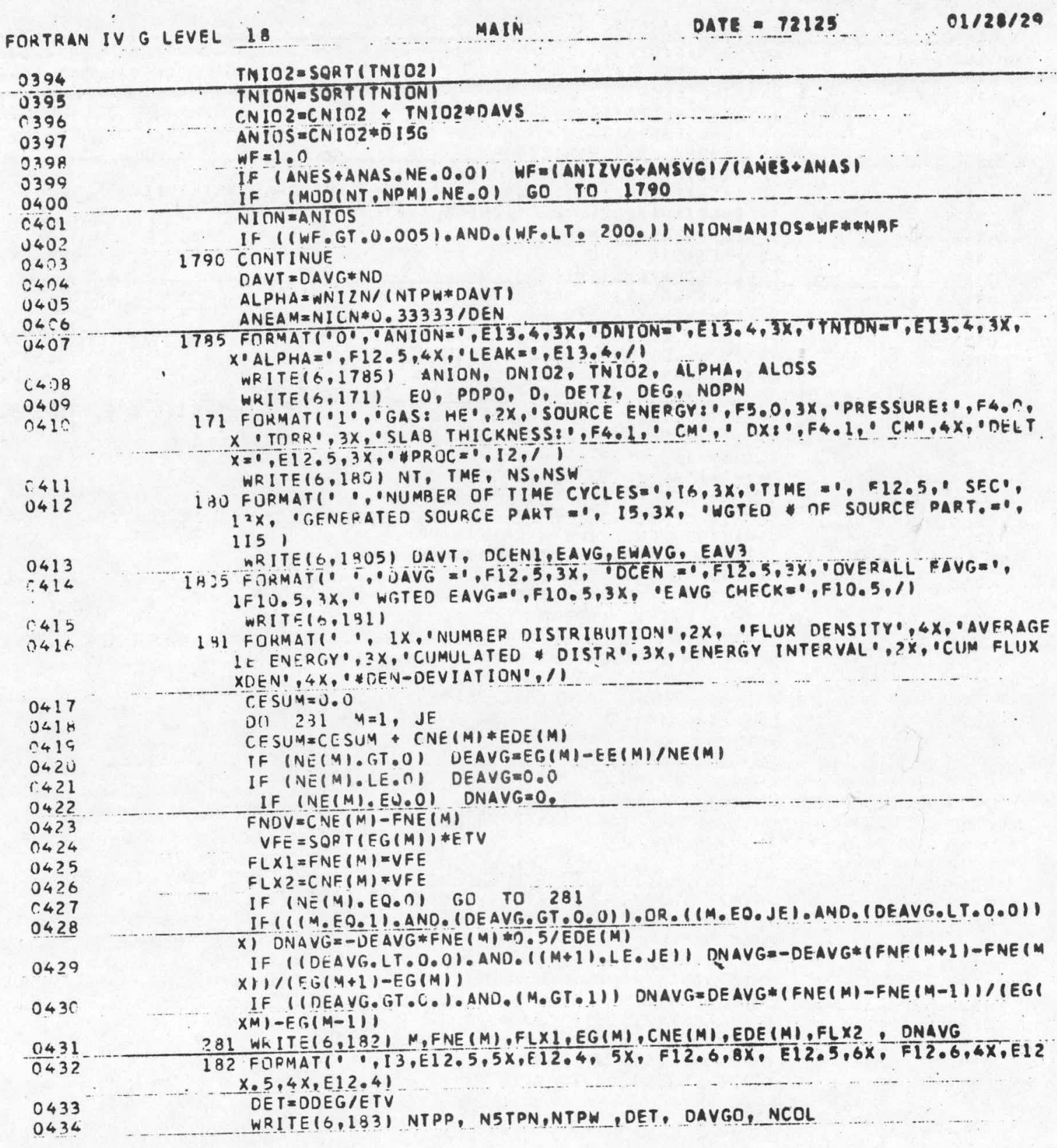




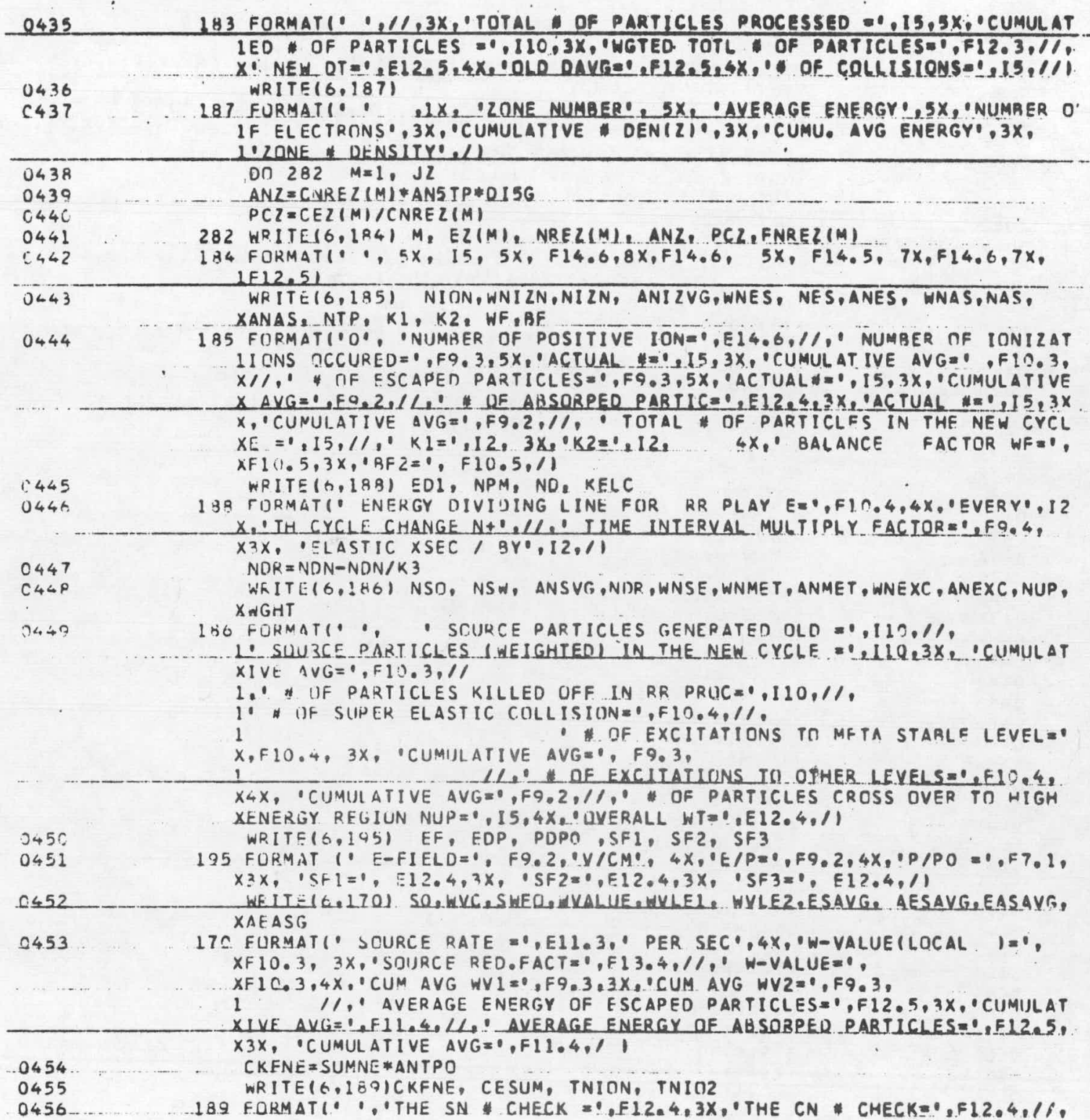




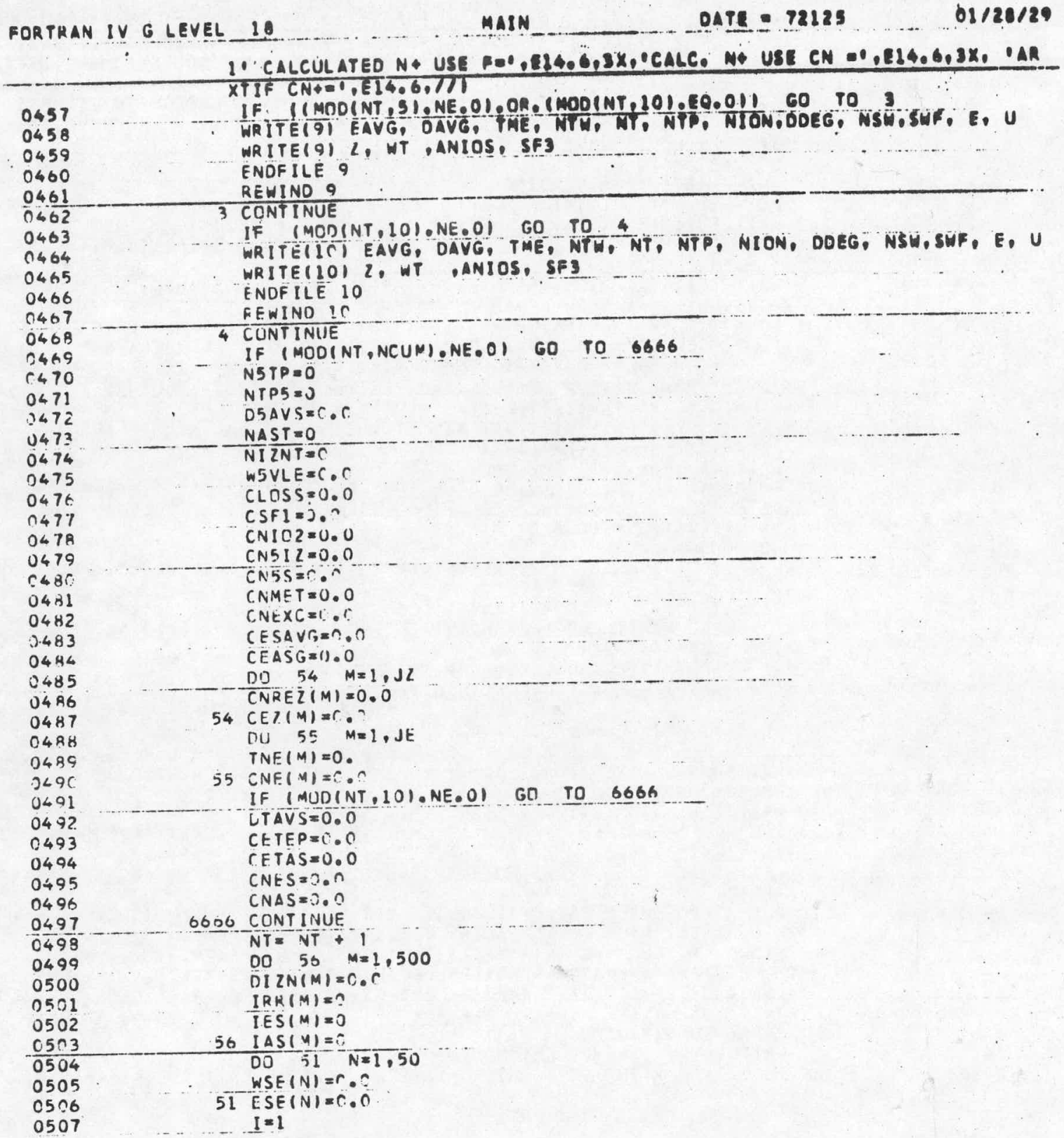


FORTRAN IV GLEVEL 18

DATE $=72125$

02128129

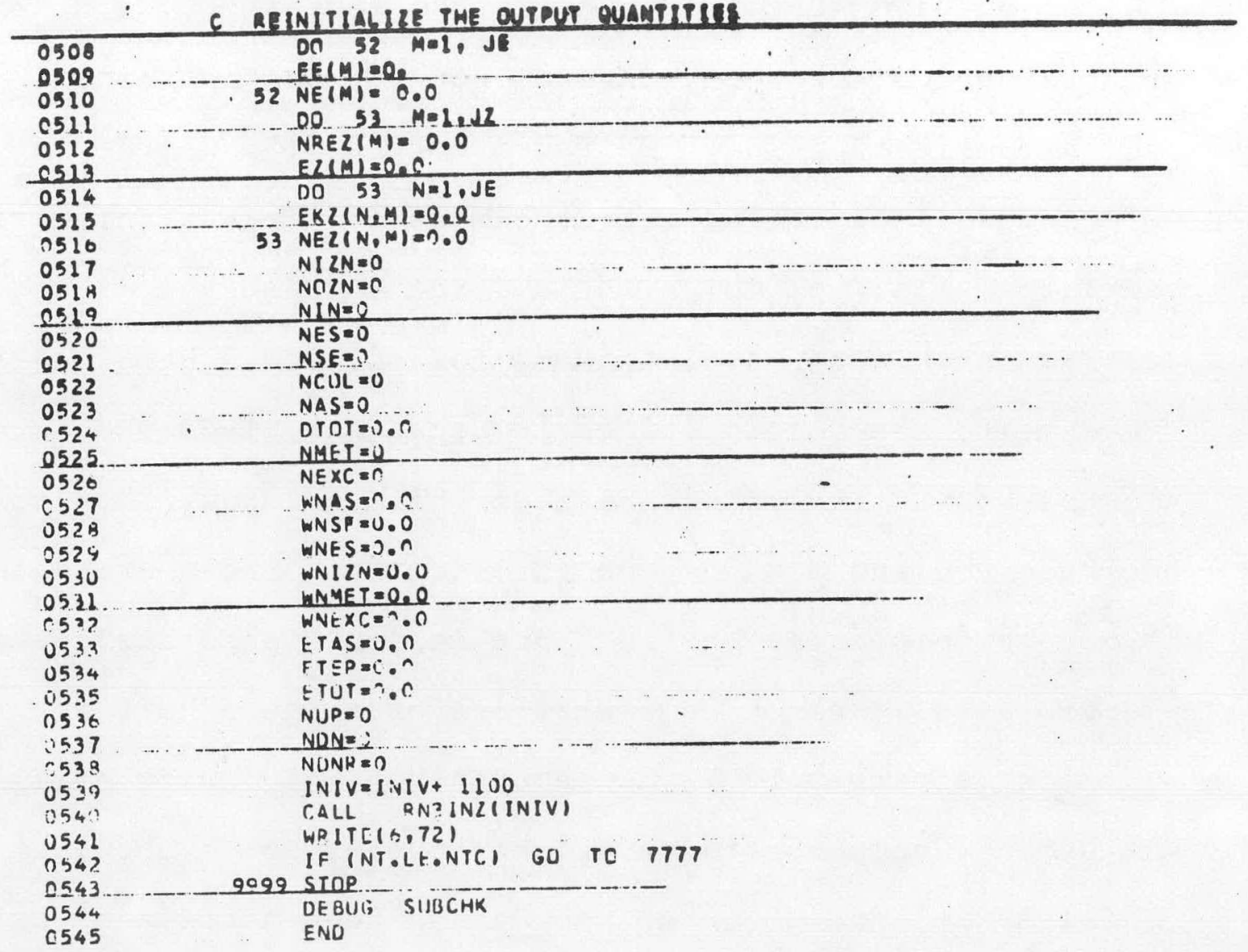


VITA

Benjamin Shaw-hu Wang was born in Inner Mongolia, China, on March 25, 1937. He is the son of Mr. C. H. Wang, the former Chinese Army General now the Congressman of the Republic of China. He received the B.S. degree in Electrical Engineering from the National Taiwan University of Taipei, Taiwan, China in 1960, he then served as an ensign in Chinese Naval Academy for one and a half years. He came to the United States in September, 1962 and enrolled in the University of Missouri at Rolla, Missouri. After he received the M.S. degree in Electrical Engineering in 1964, he joined the Westinghouse Research Laboratories and served as a research engineer for three and a half years. In September 1967, he came to the University of Illinois at Urbana-Champaign to undertake studies for a $\mathrm{Ph} . \mathrm{D}$. in . Computer Science Department. He has been a research assistant and teaching assistant in the Department of Computer Science and a research assistant in the Nuclear Engineering Program. He is the member of Kappa Mu Epsilon and Pi Mu Epsilon the two, national honor societies of mathematics. 
BIBLIOGRAPHIC DATA

SHEET

te and Subtitle

Monte Carlo Simulation of Nonlinear Radiation Induced Plasmas

7. Author(s)

Benjamin Shaw-hu Wang

9. Performing Organization Name and Address Department of Computer Science

University of Illinois at Urbana-Champaign

Urbana, Illinois 61801

12. Sponsoring Organization Name and Address

Department of Computer Science

University of Illinois at Urbana-Champaign

Urbana, Illinois 61801
3. Recipient's Accession No.

5. Report Date May, 1972

6.

8. Performing Organization Rept. NiUCDCS-R-72-519

10. Project/Task/Work Unit No.

11. Contract/Grant No.

13. Type of Report \& Period Covered Ph.D. Thesis

15. Supplementary Notes

\section{Abstracts}

A Monte Carlo simulation model for radiation induced plasmas with nonlinear properties has been developed, which employs a piecewise linearized predict-correct iterative scheme to resolve nonlinear characteristics. Several variance reduction techniques have been developed and incorporated into the model which include the antithetic variates technique. The model has been applied to the determination of electron energy distribution functions in a noble gas plasma created by $\alpha$-particle irradiation. The model can be applied to other types of complex plasma problems with nonlinear properties.

17. Key Words and Document Analysis. 17a. Descriptors

Monte Carlo Simulations, Monte Carlo Calculation of electron-energy distribution functions, Monte Carlo method for radiation induced plasmas, electron energy distribution functions in noble gas plasmas,

Monte Carlo Techniques for plasma problems,

Monte Carlo Simulation model for nonlinear plasmas.

17b. Identifiers/Open-Ended Terms

COSATI Field/Group

vailability Statement

Release unlimited

\begin{tabular}{|l|l|}
$\begin{array}{l}\text { 19. Security Class (This } \\
\text { Report) } \\
\text { UNCLASSIEIED }\end{array}$ & $\begin{array}{c}\text { 21. No of Pages } \\
166\end{array}$ \\
\hline $\begin{array}{l}\text { 20. Security Class (This } \\
\text { Page } \\
\text { UNCLASSIFIED }\end{array}$ & 22. Price \\
\hline
\end{tabular}

